University of Tennessee Health Science Center

UTHSC Digital Commons

\title{
Characteristics and Outcomes of Individuals Self-Selecting Yoga versus Physical Therapy for the Treatment of Chronic Low Back Pain
}

Dian Dowling Evans

University of Tennessee Health Science Center

Follow this and additional works at: https://dc.uthsc.edu/dissertations

Part of the Alternative and Complementary Medicine Commons, Movement and Mind-Body Therapies Commons, Nursing Commons, and the Physical Therapy Commons

\section{Recommended Citation}

Evans, Dian Dowling, "Characteristics and Outcomes of Individuals Self-Selecting Yoga versus Physical Therapy for the Treatment of Chronic Low Back Pain" (2009). Theses and Dissertations (ETD). Paper 76. http://dx.doi.org/10.21007/etd.cghs.2009.0087. 


\title{
Characteristics and Outcomes of Individuals Self-Selecting Yoga versus Physical Therapy for the Treatment of Chronic Low Back Pain
}

\begin{abstract}
Chronic low back pain (cLBP) is a significant public health problem that is difficult and costly to treat. Determining whether a patient with CLBP will benefit from a particular treatment approach is challenging, since little is understood about the patient characteristics that predict improved treatment outcomes. Understanding these characteristics could reduce treatment failures and costs of care.

This study was designed to explore the demographic, clinical and treatment- related characteristics of individuals self-selecting yoga versus physical therapy (PT) for treatment of cLBP. A growing body of research demonstrates that yoga reduces pain and improves function in persons with CLBP. However, questions remain about how people selecting yoga as a treatment for cLBP differ from persons selecting standard therapies, like PT, and whether pre-treatment differences influence treatment outcomes.

A convenience sample of 53 adults $\geq$ age 18 with cLBP $\geq 3$ months enrolling in either a 6 week modified Integral yoga program $(n=27)$ or a 6 week program of individualized PT $(n=26)$ participated in the study. Data were collected at baseline and at 6 and 12 weeks after completion of treatment. Study variables included disability (Roland Morris Disability Questionnaire), depression (Beck Depression Inventory-II), health status (RAND Short-Form 36-Item Health Survey 1.0 Questionnaire), fear of movement (Tampa Scale of Kinesiophobia), pain self-efficacy, pain bothersomeness, pain medication use, reasons for treatment choice and satisfaction with care. Group differences were compared using non-parametric statistics.

The findings revealed no significant differences between the groups in duration of CLBP $(9.02 \pm 9.29$ years), worst pain in the past 6 months $(8.05 \pm 1.93$ on a $0-10$ pain bothersomeness scale), age ( $50.69 \pm$ 15.56 years), gender ( $66 \%$ female) or education ( $16 \pm 3.05$ years). However, PT participants were more likely to be nonwhite, earning $\leq \$ 30,000 / \mathrm{yr}$ and selecting PT based on healthcare provider referral or insurance coverage. Yoga participants were more likely to be gainfully employed, earning $\geq \$ 70,000 / y r$ and selecting yoga based on personal research. Neither group was depressed at baseline, but PT participants were significantly more disabled, had lower health status, greater pain and twice the pain medication use compared to yoga participants at baseline.
\end{abstract}

At the conclusion of 6 weeks of treatment, both groups had significant improvements in disability and health status with fewer days in pain. PT participants who showed significantly greater pain symptoms at baseline had greater reductions in pain at 6 and 12 weeks compared to yoga participants. At 12 weeks the groups had equivalent pain levels, demonstrating persistent treatment benefits with few adverse effects and high satisfaction with care. Cost was cited as a reason for early termination of treatment for PT participants but was not an issue for yoga participants. The improved outcomes and group differences support the effectiveness of both treatments in reducing pain and disability. The similarities between the groups at 12 weeks and differences in total cost of care suggest the need for further research to examine the long-term costs and benefits of yoga versus PT for treatment of cLBP.

\section{Document Type}

Dissertation

Degree Name

Doctor of Philosophy (PhD) 


\section{Program}

Nursing

Research Advisor

Michael Carter, DNSc, FAAN

\section{Keywords}

Yoga, physical therapy, back pain

\section{Subject Categories}

Alternative and Complementary Medicine | Medicine and Health Sciences | Movement and Mind-Body Therapies | Nursing | Physical Therapy | Rehabilitation and Therapy

\section{Comments}

Six month embargo expired October 2009 
Characteristics and Outcomes of Individuals Self-Selecting Yoga versus Physical Therapy for the Treatment of Chronic Low Back Pain

\author{
A Dissertation \\ Presented for \\ The Graduate Studies Council \\ The University of Tennessee \\ Health Science Center
}

\author{
In Partial Fulfillment \\ Of the Requirements for the Degree \\ Doctor of Philosophy \\ From The University of Tennessee
}

By

Dian Dowling Evans

May 2009 
Copyright (C) 2009 by Dian Dowling Evans

All rights reserved 


\section{DEDICATION}

This dissertation is dedicated to my mentors, friends and fellow yoga practitioners, who inspired and lent support to me as I began and carried out this dissertation process;

to my husband Jeff, who encouraged, fed and nurtured me; and to my children and grandchildren,

who enable me to see the world in new ways and risk new ideas. 


\section{ACKNOWLEDGEMENTS}

I want to thank and express my appreciation to Dr. Michael Carter for his tremendous support of creativity and for his guidance, direction and encouragement during the preparation, investigation and writing phases of this dissertation research. I also want to thank my committee members, Dr. James Pruett, Dr. Margaret Hartig and Dr. Patricia Cowan, for their assistance and support in identifying important issues that improved my interpretation of this study's findings.

An expression of special appreciation goes to committee member Dr. Richard Panico for the hours that he spent sharing his knowledge of classical yoga philosophy and of Integral yoga methods. His encouragement allowed me to conduct this dissertation project at the Athens Regional Medical Center Mind-Body Institute, where he serves as Medical Director. A thank you goes to Dr. Chris Doerr, Medical Director of the Physician Back and Neck Clinic in Athens, Georgia, for sharing his knowledge of exercise physiology and innovative exercise approaches for treating chronic low back pain (cLBP) and for encouraging me to conduct this research in his clinic. I also appreciate the efforts of Todd Story, P.T., Director of the Athens Regional Medical Center Rehabilitation Department, for his support in allowing me to conduct this study in the physical therapy clinic at Athens Regional Medical Center. I also thank the therapists and staffs of both physical therapy facilities for their assistance in recruitment efforts and in conducting the intervention phase of this research.

Special appreciation of my friend and colleague, Dr. Laura Kimble, is acknowledged. The support and hours of research guidance she offered during the evolution of this dissertation research were invaluable..

Finally, I want to thank Jennifer Morlock, P.T. and Helene Herndon, R.Y.T. for being wonderful, supportive, volunteer research assistants whose dedication and organizational skills throughout the investigation phase of this dissertation facilitated its completion. Special thanks to Manjula Spears, R.Y.T. my first yoga teacher who helped me put the findings of this project in perspective. 


\begin{abstract}
Chronic low back pain (cLBP) is a significant public health problem that is difficult and costly to treat. Determining whether a patient with cLBP will benefit from a particular treatment approach is challenging, since little is understood about the patient characteristics that predict improved treatment outcomes. Understanding these characteristics could reduce treatment failures and costs of care.

This study was designed to explore the demographic, clinical and treatmentrelated characteristics of individuals self-selecting yoga versus physical therapy (PT) for treatment of cLBP. A growing body of research demonstrates that yoga reduces pain and improves function in persons with cLBP. However, questions remain about how people selecting yoga as a treatment for cLBP differ from persons selecting standard therapies, like PT, and whether pre-treatment differences influence treatment outcomes.
\end{abstract}

A convenience sample of 53 adults $\geq$ age 18 with cLBP $\geq 3$ months enrolling in either a 6 week modified Integral yoga program $(n=27)$ or a 6 week program of individualized PT $(n=26)$ participated in the study. Data were collected at baseline and at 6 and 12 weeks after completion of treatment. Study variables included disability (Roland Morris Disability Questionnaire), depression (Beck Depression Inventory-II), health status (RAND Short-Form 36-Item Health Survey 1.0 Questionnaire), fear of movement (Tampa Scale of Kinesiophobia), pain self-efficacy, pain bothersomeness, pain medication use, reasons for treatment choice and satisfaction with care. Group differences were compared using non-parametric statistics.

The findings revealed no significant differences between the groups in duration of cLBP $(9.02 \pm 9.29$ years $)$, worst pain in the past 6 months $(8.05 \pm 1.93$ on a $0-10$ pain bothersomeness scale), age (50.69 \pm 15.56 years), gender ( $66 \%$ female) or education (16 \pm 3.05 years). However, PT participants were more likely to be nonwhite, earning $\leq$ $\$ 30,000 / y r$ and selecting PT based on healthcare provider referral or insurance coverage. Yoga participants were more likely to be gainfully employed, earning $\geq \$ 70,000 / \mathrm{yr}$ and selecting yoga based on personal research. Neither group was depressed at baseline, but PT participants were significantly more disabled, had lower health status, greater pain and twice the pain medication use compared to yoga participants at baseline.

At the conclusion of 6 weeks of treatment, both groups had significant improvements in disability and health status with fewer days in pain. PT participants who showed significantly greater pain symptoms at baseline had greater reductions in pain at 6 and 12 weeks compared to yoga participants. At 12 weeks the groups had equivalent pain levels, demonstrating persistent treatment benefits with few adverse effects and high satisfaction with care. Cost was cited as a reason for early termination of treatment for PT participants but was not an issue for yoga participants. The improved outcomes and group differences support the effectiveness of both treatments in reducing pain and disability. The similarities between the groups at 12 weeks and differences in 
total cost of care suggest the need for further research to examine the long-term costs and benefits of yoga versus PT for treatment of cLBP. 


\section{TABLE OF CONTENTS}

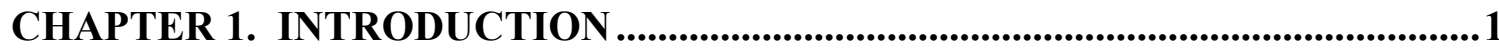

Background 1

Purpose and Aims ................................................................................... 4

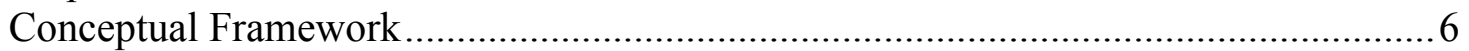

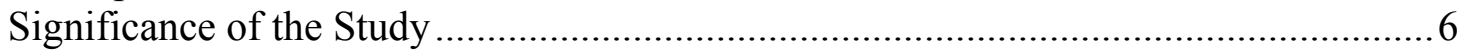

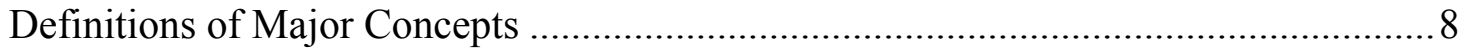

Assumptions 9

CHAPTER 2. REVIEW OF LITERATURE ............................................................11

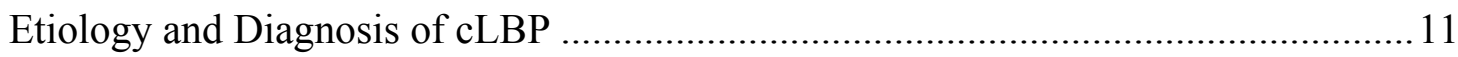

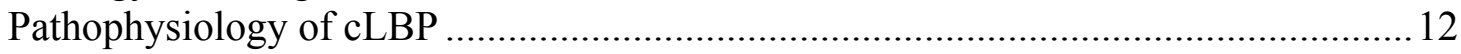

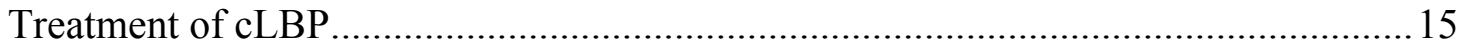

Medical, Chiropractic and Manual Therapies ............................................... 15

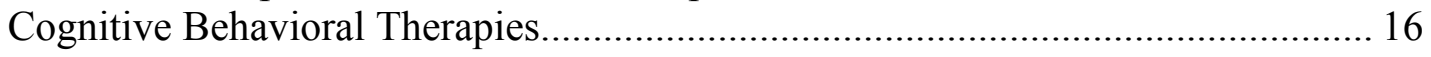

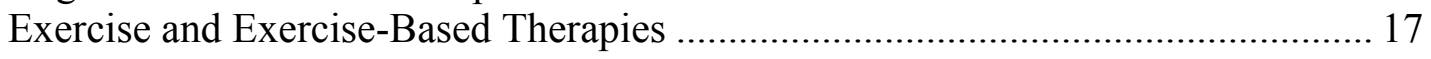

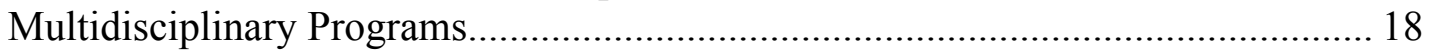

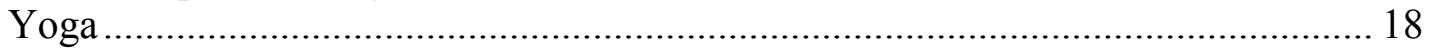

Core Domains and Clinical Practice Guidelines for Treatment of cLBP ...................22

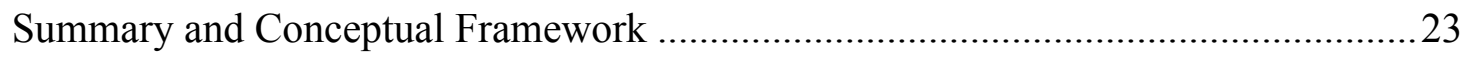

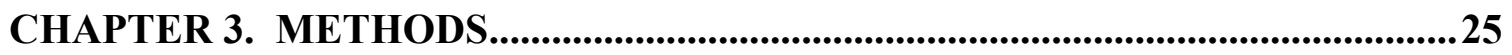

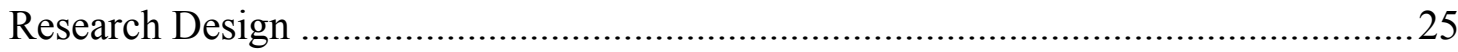

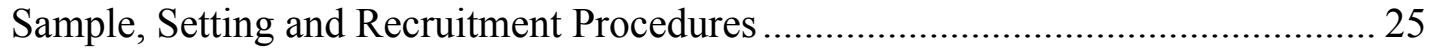

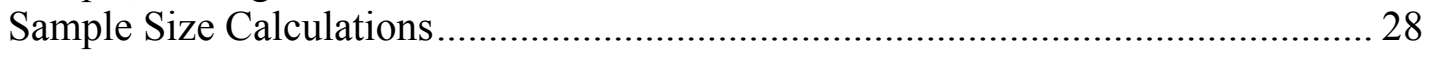

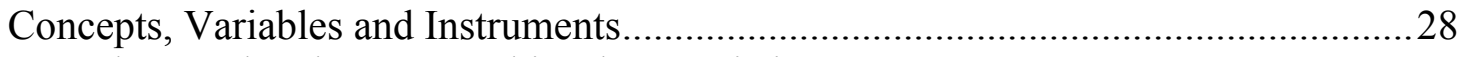

Background and Demographic Characteristics ................................................ 28

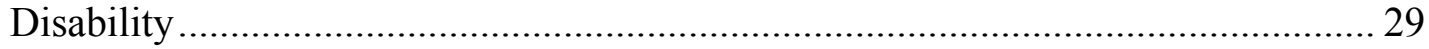

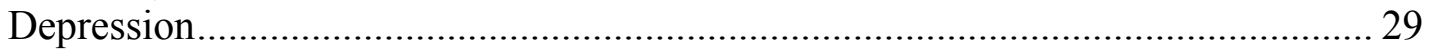

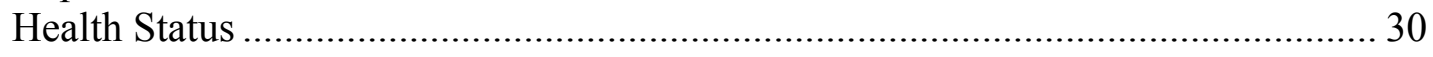

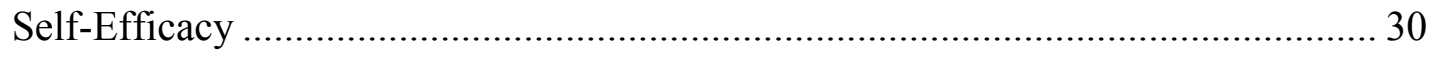

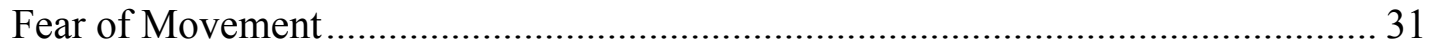

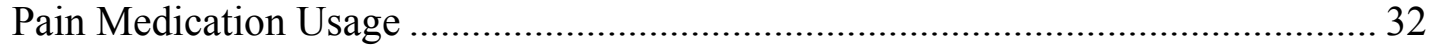

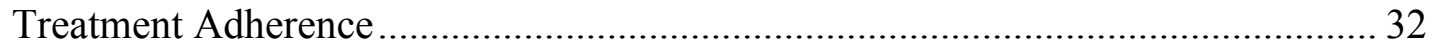

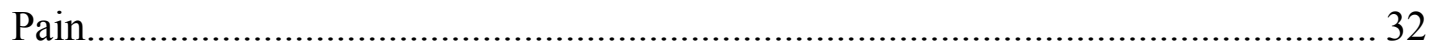

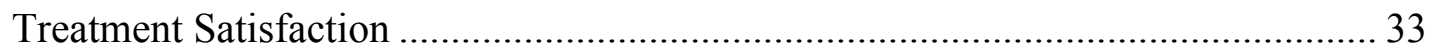

Conceptual Model with Study Variables and Variable Relationships.........................33

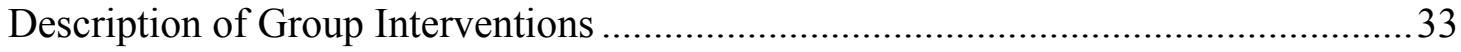

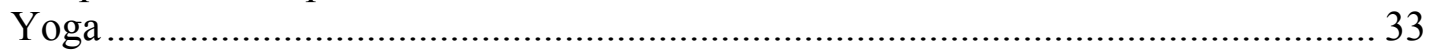

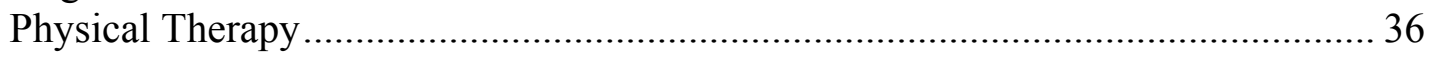

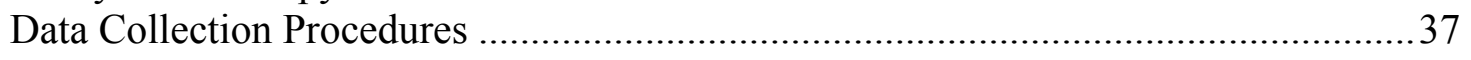

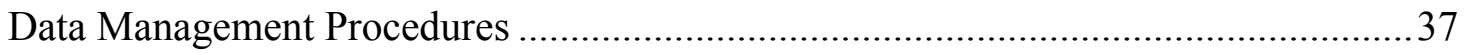

Data Analysis39 


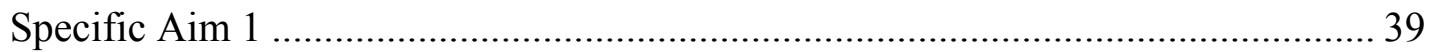

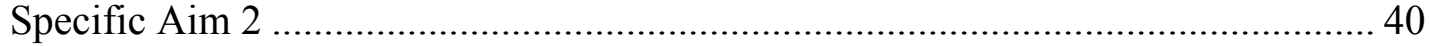

Consideration of Human Subjects ............................................................................. 41

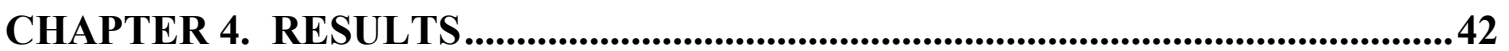

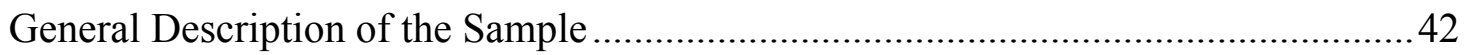

Aim 1-To Describe Group Differences at Baseline ...................................................42

Aim 2 - To Compare Treatment Outcomes...............................................................45

CHAPTER 5. DISCUSSION...........................................................................................59

Aim 1-Baseline Characteristics and Differences ..................................................59

Aim 2-To Compare Treatment Outcomes............................................................61

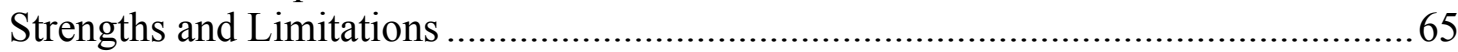

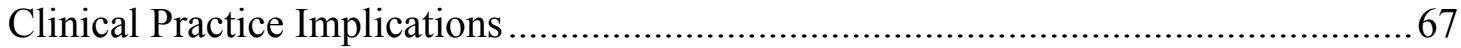

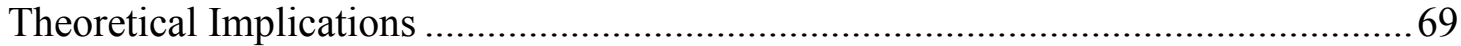

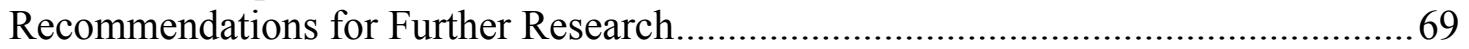

Conclusions 70

LIST OF REFERENCES .................................................................................................

APPENDIXES ....................................................................................................................... 80

Appendix A. Survey Consent Form.......................................................................... 81

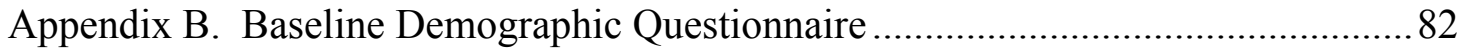

Appendix C. Roland Morris Disability Questionnaire ……………………………....... 86

Appendix D. RAND Short-Form 36-Item Health Survey 1.0 Questionnaire..............87

Appendix E. Back Pain Self-Efficacy Scale ..............................................................94

Appendix F. Tampa Scale of Kinesiophobia ...........................................................98

Appendix G. Weekly Practice and Pain Log ......................................................... 101

Appendix H. Pain Characteristics Questionnaire ........................................................ 102

Appendix I. Short Term Yoga Program Follow-up Questionnaire ..............................103

Appendix J. Short Term Physical Therapy Follow-up Questionnaire.........................105

Appendix K. Long Term Yoga and Physical Therapy Follow-up Questionnaire ..... 107

Appendix L. Back Yoga Sequence ............................................................................109 


\section{LIST OF TABLES}

Table 3.1 Assessment Procedures........................................................................ 38

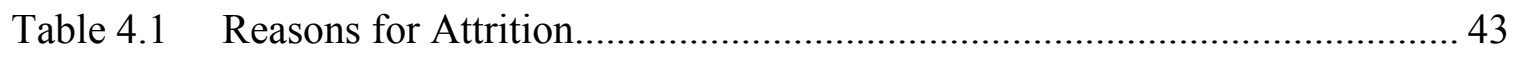

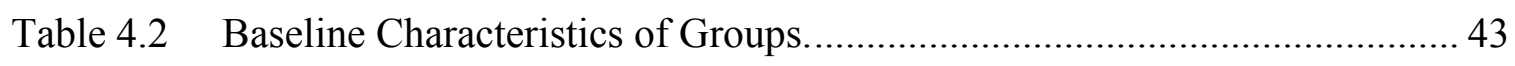

Table 4.3 Pre-Treatment Reasons for Choosing PT or Yoga. .................................. 44

Table 4.4 Pre-Treatment Expectations................................................................ 44

Table 4.5 Pre-Treatment Group Comparisons of Psychological Variables................. 46

Table 4.6 Pre-Treatment Group Comparisons of Health Status and Disability........... 46

Table 4.7 Pre-Treatment Differences in Pain-Related Variables. .............................. 47

Table 4.8 Within Group Changes on Psychological Measures at 6 Weeks.................. 48

Table 4.9 Between Group Psychological Change Score Differences at 6 Weeks....... 48

Table 4.10 Yoga within Group Disability and Health Status Differences at 6 Weeks... 50

Table 4.11 PT within Group Disability and Health Status Differences at 6 Weeks...... 50

Table 4.12 Between Group Baseline to 6 week Treatment Outcome Differences in Disability and Health Status. ............................................................... 51

Table 4.13 Group Differences in Pain Medication Usage at 6 and 12 Weeks. ............. 55

Table 4.14 Between Group Treatment Outcome Differences at 6 Weeks..................... 56

Table 4.15 Between Group Treatment Outcome Differences at 12 Weeks................... 58 


\section{LIST OF FIGURES}

Figure 1.1 The Disablement Model. ..........................................................................

Figure 1.2 Modified Disablement Model with Study Variables and Variable

Relationships. .7

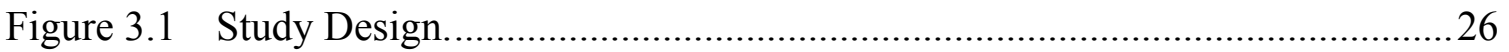

Figure 3.2 Modified Disablement Model with Study Variables, Instruments and Concept Relationships...................................................................... 34

Figure 4.1 Group Differences in Average Number of Days in Pain in the Past 2 Weeks at Baseline, 6 and 12 Weeks...................................................51

Figure 4.2 Number of Days in the Past Month with Reduced Activity Secondary

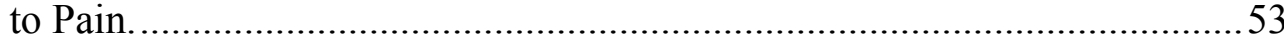

Figure 4.3 Average Pain Bothersomeness in the Past 2 Weeks.................................53

Figure 4.4 Worst Pain Bothersomeness in the Past Month ..........................................53 


\section{LIST OF ABBREVIATIONS}

$\begin{array}{ll}\text { AHRQ } & \text { Agency for Health Research and Quality } \\ \text { BDI-II } & \text { Beck Depression Inventory-II } \\ \text { BPSES } & \text { Back Pain Self-Efficacy Scale } \\ \text { cLBP } & \text { Chronic Low Back Pain } \\ \text { CPG } & \text { Clinical Practice Guidelines } \\ \text { DSM-III } & \text { Diagnostic and Statistical Manual of Mental Disorders-III } \\ \text { MET } & \text { Metabolic Equivalent } \\ \text { MMPI } & \text { Minnesota Multiphasic Personality Inventory } \\ \text { PT } & \text { Physical Therapy } \\ \text { RAND SF-36 1.0 } & \text { RAND Short-Form 36-Item Health Survey 1.0 } \\ \text { RCT } & \text { Randomized Control Trial } \\ \text { RMDQ } & \text { Roland Morris Disability Questionnaire } \\ \text { SPSS } & \text { Statistical Package for the Social Sciences } \\ \text { TSK } & \text { Tampa Scale of Kinesiophobia }\end{array}$




\section{CHAPTER 1. INTRODUCTION}

\section{Background}

Low back pain is a very common public health problem affecting approximately $80 \%$ of adults in the United States during their lifetime (1997). Back pain is the second most common reason for visits to healthcare practitioners (Heffernan, 2001) with annual prevalence rates ranging from $15 \%$ to $45 \%$ (Andersson, 1999). Although most cases of back pain are mild and resolve within 6-12 weeks (Ehrlich, 2003), 15\% of individuals develop chronic symptoms or a pattern of recurring symptom exacerbation associated with limitations in daily function and mobility (Carey, Garrett, Jackman, \& Hadler, 1999; Deyo \& Weinstein, 2001). These limitations lead to other problems such as physical deconditioning (Puetz, O'Connor, \& Dishman, 2006), loss of muscle strength and joint flexibility (Langevin \& Sherman, 2007) and psychological distress (Deyo \& Weinstein, 2001; Frost, Lamb, Klaber, Moffett, Fairbank, \& Moser, 1998).

Chronic low back pain (cLBP) is a complex condition that is difficult to treat for several reasons. First, back pain exacerbations and recurrences are neither predictable (Kovacs et al., 2004) nor preventable (U.S. Preventive Services Task Force, 2004). This unpredictable natural course of cLBP complicates evaluating treatment outcomes (Carey et al., 1996).

Second, despite numerous investigations evaluating associations between diagnostic imaging results, physical examination findings, functional disability and the subjective experience of cLBP, predictable relationships have not been identified (Heffernan, 2001). Persons with cLBP often lack a specific etiology for their pain, and even during periods of remission may experience low levels of physical and psychological distress that adversely affect their overall health status, physical functioning and ability to meet work and other social responsibilities (Ricci et al., 2006; Weiner, 2008).

A third problem is that existing evidence used to predict acceptable, beneficial treatment approaches based on patient characteristics and physical examination findings is inconclusive. Several cLBP patient classification and treatment predictive models have been proposed (Childs et al., 2004; Riddle, 1998; Thomas et al., 1999); however, clinical trials evaluating the effectiveness of these models have failed to identify either the specific patient or treatment characteristics that predict or correlate with improvements in pain and function (Deyo \& Weinstein, 2001; Marshall \& Murphy, 2008). Similarly, investigations exploring associations between pain, quality of life, function and disability have reported inconsistent results (Kovacs et al., 2004).

Current evidence strongly suggests that psychological factors, such as anxiety and depression (Ehrlich, 2003), fear of movement (Grotle, Vollestad, Veierod, \& Brox, 2004; Vlaeyen, Kole-Snijders, Boeren, \& van Eek, 1995), self-efficacy (Asghari \& Nicholas, 2001) and satisfaction with care (Butler \& Johnson, 2008; Kalauokalani, 
Cherkin, Sherman, Koepsell, \& Deyo, 2001) influence the course of back pain to a greater extent than do physical factors. However, determining the most effective treatment approach based on psychological factors is also unclear. These gaps in knowledge contribute to high treatment failure rates and high back treatment costs of care. This study was designed to examine clinical and demographic characteristics of two groups of individuals with cLBP who selected either physical therapy or yoga for treatment of back pain and to compare treatment outcomes. The purpose of this study was to improve the understanding of how pre-existing factors may influence treatment success or failure.

To improve treatment outcomes and reduce costs of care, the Agency for Health Research and Quality (AHRQ) published evidence-based clinical practice guidelines (CPG's) for the treatment of cLBP in 1999 for the Veterans Administration (Jackson \& Browning, 2005). The guidelines emphasize that the primary goals in treating cLBP are to control pain, improve physical function and to reduce psychological distress (Koes, van Tulder, \& Thomas, 2006; van Tulder, Furlan, \& Gagnier, 2005). Adherence to CPGs has been shown to improve clinical outcomes and reduce costs of care (Feuerstein, Hartzell, Rogers, \& Marcus, 2006; Webster, Courtney, Huang, Matz, \& Christiani, 2005).

Yet studies of healthcare provider treatment decision making have found that healthcare provider practice style, which may be based upon past clinical experiences rather than evidenced-based guidelines, can influence how treatment decisions are made (Webster, Courtney, Huang, Matz, \& Christiani, 2006). Treatment decisions based on physician preference or practice style can significantly influence back treatment success or failure when patient expectations are not considered in treatment selection (Butler \& Johnson, 2008; Eisenberg et al., 2007) and when evidence-based guidelines are not used to guide care (Webster, Courtney, Huang, Matz, \& Christiani, 2005). Failure to consider patient-related factors or to adhere to the CPGs also influences costs of care (Chou, 2005).

Another factor in cLBP clinical decision-making that can lead to increased costs is basing treatment decisions on economic considerations, including patient resources and insurance reimbursement allowances rather than other patient-related factors or evidencebased guidelines. Treatment decisions based on economics contributes to increased health care costs when treatments are discontinued as resources become depleted before patients have time to achieve treatment benefits and when patients discontinue due to dissatisfaction with care. Costs may also increase from adverse iatrogenic side effects from invasive procedures or from medications, including GI bleeding and narcotic addiction (Ehrlich, 2003; Heffernan, 2001).

In 2002 the annual U.S. health care expenditures for back pain-related costs were estimated at $\$ 91$ billion or $1 \%$ of the U.S. gross national product. From this, $\$ 14.1$ billion was spent on prescription drugs and $\$ 35.5$ billion was spent for physician and outpatient services (Luo, Pietrobon, Sun, Liu, \& Hey, 2004). Persons with cLBP were also found to incur 1.6 times higher health care costs annually compared to individuals without cLBP (Luo et al., 2004) and to experience more absenteeism and lost wages (Ricci et al., 2006). 
Costs to employers due to lost productivity and to insurers due to increased health care expenditures were also substantial (Luo et al., 2004; Ricci et al., 2006).

In 2007 the American College of Physicians and American Pain Society recommended updating the CPG guidelines for management of cLBP based on new evidence obtained from systematic reviews and meta-analyses of high quality back treatment clinical trials (Chou et al., 2007). The recommendations support the continued use of conservative approaches in managing cLBP, including exercise to strengthen muscles and restore physical conditioning and cognitive behavioral therapies to reduce psychological distress. The recommendations also added the use of yoga in treating cLBP based on evidence of yoga's moderate to strong effect in reducing pain and improving function (Sherman, Cherkin, Erro, Miglioretti, \& Deyo, 2005; Williams et al., 2005).

The use of yoga has been increasing among U.S. adults since the 1970s. A national survey conducted in the U.S in 1997 of adults between the ages of 34-53 found that approximately 7.5 million adults, or $3.7 \%$, had used yoga at least once in the past year (Saper, Eisenberg, Davis, Culpepper, \& Phillips, 2004). Among those surveyed, $64 \%$ reported using yoga for wellness, $48 \%$ for specific health conditions and $21 \%$ for relief of back pain. Additionally, $90 \%$ of users reported yoga was helpful and $76 \%$ reported no increased costs for yoga use. A more recent survey conducted in 2002 found that the number of yoga users had increased to 10.4 million adults, or $5.1 \%$, making yoga the fastest growing of all complementary and alternative medical approaches used in the U.S. (Barnes, Powell-Griner, McFann, \& Nahin, 2004).

Research evidence supporting yoga use for treatment of chronic conditions, including cLBP, is also increasing. Yoga has been found to be easily modified and adapted for individuals with physical limitations (Bastille \& Gill-Body, 2004; Michels, Edwards, Salstrom, Spears, \& Panico, 2006; Oken et al., 2004), including elderly adults (DiBenedetto et al., 2005; Greendale, McDivit, Carpenter, Seeger, \& Huang, 2002) and those with cLBP (Galantino et al., 2004; Sherman, Cherkin, Erro, Miglioretti, \& Deyo, 2005; Williams et al., 2005). In addition, yoga has been found to reduce anxiety and depression (Michalsen et al., 2005; Michels, Edwards, Salstrom, Spears, \& Panico, 2005; Netz \& Lidor, 2003; Woolery, Myers, Sternlieb, \& Zeltzer, 2004) and to increase selfefficacy (Lee, Mancuso, \& Charlson, 2004; Waelde, Thompson, \& Gallagher-Thompson, 2004) which are associated with improved back treatment outcomes (Asghari \& Nicholas, 2001; Cherkin, Deyo, Street, \& Barlow, 1996). Yoga effects on back pain and function result within 12-16 hours of treatment and are found to persist following completion of therapy (Galantino et al., 2004; Sherman et al., 2005; Williams et al., 2005).

Criticisms of yoga research are that many published studies lack rigor and randomization, are under-powered, and consist of small sample sizes (Graves, Krepcho, \& Mayo, 2004). A 2004 systematic review of published yoga research studies found 141 clinical trials using yoga or yoga methods, with the majority published since 1990 (Khalsa, 2004), but only $39.8 \%$ of published studies were randomized control trials 
(RCTs), 48.1\% had uncontrolled designs and 12.2\% used non-randomized control groups (Khalsa, 2004). Other concerns include poorly described yoga methodologies, making cross-comparisons of studies challenging, and variations in control groups, making yoga effects difficult to interpret (Jacobs et al., 2004; Raub, 2002). Another concern is that yoga users and research participants are typically white, middle-aged, college-educated females, which limits generalization of research findings to other populations (Birdee et al., 2008). Despite these concerns, the evidence supporting yoga use in treating chronic musculoskeletal problems is promising.

Yoga is generally taught within a group class setting or through private instruction. The average cost of a yoga class series throughout the U.S. ranges from $\$ 75.00$ - \$200.00 for a 6-12 week series. Private instruction can range from $\$ 50-\$ 200.00$ per 90 minute session. Yoga also can be self-taught and practiced at home using videotapes, DVD's and books. When compared to the costs of physician office visits, prescription drugs, invasive procedures and conventional physical and psychological therapies, yoga is beneficial and economical for consumers. Although mild adverse effects from yoga have been reported, including transient joint stiffness and muscle soreness, these appear to resolve quickly with continued practice ((Dowling, Carter, \& Panico, 2008); Sherman et al., 2005; Williams, Petronis, Smith, Goodrich, Wu, Ravi, Doyle, Juckett et al., 2005).

Barriers to yoga use in the treatment of cLBP include a lack of access to teachers trained in yoga therapeutics and a lack of awareness and understanding by healthcare providers about the use of yoga for treatment of cLBP. In addition, healthcare providers may not be aware of the current evidence recommending the use of yoga in the treatment of cLBP or of the types of patients who may benefit from yoga use. Improving healthcare provider knowledge of yoga benefits could save billions of dollars a year in the U.S. in cLBP treatment costs.

The need for further research to evaluate cost effective beneficial treatments for cLBP is clear. The emerging evidence demonstrating that yoga is beneficial and also cost effective suggests that continued investigation of yoga effects in the treatment of cLBP is needed. Additionally, questions remain about how people selecting yoga for treatment differ from people selecting standard therapies, like physical therapy, and whether pretreatment differences affect treatment outcomes. The present study was designed to explore these questions.

\section{Purpose and Aims}

The purpose of this study was to compare the characteristics of individuals selfselecting yoga with those self-selecting physical therapy for the treatment of cLBP and to examine differences in treatment outcomes between these groups. This study had two major aims. The variables and instruments used in this study were based on the core measures recommended when conducting clinical back treatment research (Deyo et al., 
1998) and which have been used by previous yoga cLBP researchers. This facilitates comparison of this study's findings with other yoga and clinical back treatment research. Study aims and associated research questions are as follows:

- Aim 1. To compare pre-existing demographic and clinical characteristics of individuals selecting yoga or physical therapy.

1.1. What are the differences between the groups in the following demographic characteristics: (a) gender, (b) age, (c) race, (d) income, (e) educational level, (f) marital status and (g) employment status?

1.2. What are the baseline differences between the groups on the psychological variables (a) depression (Beck Depression Inventory [BDI-II]), (b) pain self-efficacy (Back Pain Self-Efficacy Scale [BPSES]) and (c) fear of movement (Tampa Scale of Kinesiophobia [TSK])?

1.3 What are the baseline differences between the groups in functional disability (Roland Morris Disability Questionnaire [RMDQ])?

1.4 What are the baseline differences between the groups in health status (RAND SF-36 1.0 general health, bodily pain, social function and physical function subscales)?

1.5 What are the baseline differences between the groups in pain-related variables considering (a) total duration of cLBP in weeks(b) pain frequency (days in pain per week), (c) pain bothersomeness (worst and average), (d) average pain medication usage (doses per week) and (e) reduced activity related to pain (days per week)?

1.6 What are the baseline differences between the groups in treatment-related variables considering treatment expectations and reason for treatment selection?

- Aim 2. To compare treatment outcomes of yoga and physical therapy groups.

2.1 What are the differences between the groups on psychological outcomes at 6 weeks (BDI-II, BPSES and TSK)?

2.2 What are the differences between the groups in functional disability at 6 weeks (RMDQ)?

2.3 What are the differences between the groups in health status at 6 weeks (RAND SF-36 1.0 general health, bodily pain, social function and physical function subscales)?

2.4 What are the differences between the groups in pain-related variables at 6 and 12 weeks considering (a) average number of days in pain in past 2 weeks, (b) average number of days with activity limitation secondary to pain, (c) average pain bothersomeness in past 2 weeks and worst pain bothersomeness in the past month and (d) pain medication usage?

2.5 What are the differences between the groups in treatment-related variables at 6 and 12 weeks considering (a) minutes per week of home practice, (b) total duration of treatment in hours during the intervention period, (c) treatment satisfaction and (d) treatment benefits? 


\section{Conceptual Framework}

The Disablement Model by Verbrugge and Jette (1994) was used as the conceptual framework for this study. This model describes the factors associated with the progression to disability. Reducing disability by improving physical and psychological functioning is one of the major goals in treating cLBP. The Disablement Model is shown in Figure 1.1 (Deyo et al., 1998).

According to Verbrugge and Jette (1994), disability is defined as an impaired ability to perform one's social roles appropriate to age and gender. The progression to disability results when social and environmental demands exceed an individual's capacity to perform social roles and functions. Capacity factors include social support, positive therapeutic expectations, high self-efficacy, positive affect and beneficial treatment effects. Factors associated with capacity mitigate functional limitations, thus reducing the likelihood of disability. Demand factors consist of individual, social and environmental barriers that interfere with a person's capacity to perform expected social roles and functions. Demand factors can include economic burdens, adverse working conditions, lack of social support and psychological distress.

Functional limitations also may be influenced by demographic and co-morbid risk factors such as age, socioeconomic status and gender (Thomas et al., 1999), emotional distress and depression (Brage, Sandanger, \& Nygard, 2007), pain characteristics (Cherkin, Deyo, Street, \& Barlow, 1996), fear-avoidance behaviors (Marshall \& Murphy, 2008) and medical co-morbidities such as obesity and physical deconditioning (Verbrugge \& Jette, 1994). The dynamic interaction between capacity, demand and functional limitation factors lead to either adaptive functioning or to disability.

Figure 1.2 includes a modification of the Disablement Model to include the variables and predicted concept relationships explored in this study. Permission to modify the model was granted by the author (Alan Jette, personal communication, October 1, 2008).

\section{Significance of the Study}

This study contributes to the aims of nursing scholarship and research by adding to knowledge benefiting clinical practice and healthcare provider education in the treatment of a major public health problem. Information gained from this study also has healthcare resource and economic implications.

Chronic low back pain is a significant public health problem with substantial costs to individuals and society. People with cLBP whose treatments fail often experience increased psychosocial stress and economic burden that can interfere with their physical and psychological health status (Deyo \& Weinstein, 2001). Back treatment failures also 


\section{Capacity}

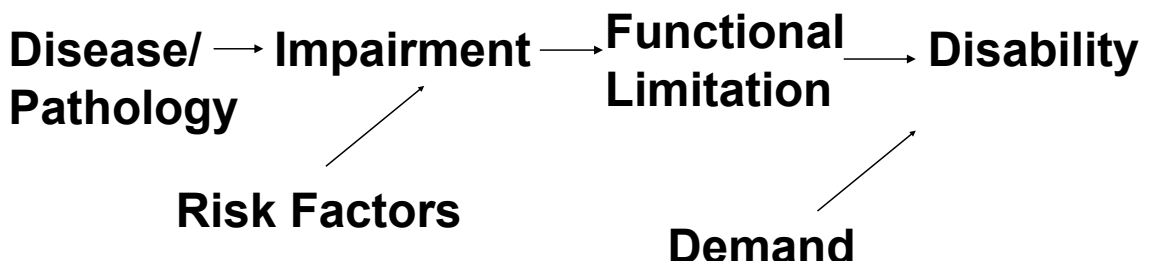

Figure 1.1 The Disablement Model.

Reprinted from Verbrugge, L. M. \& Jette, A. M. (1994). The disablement process. Social Science and Medicine, 38(1), 1-14, with permission from Elsevier.

$\begin{array}{ll}\text { Capacity } & \text { Improved } \\ \text { Back pain self-efficacy } & \text { Therapeutic } \\ \text { Treatment adherence } & \text { Outcomes } \\ \text { Medication use } & \text { Satisfaction with care } \\ \text { Positive treatment expectations } & \end{array}$

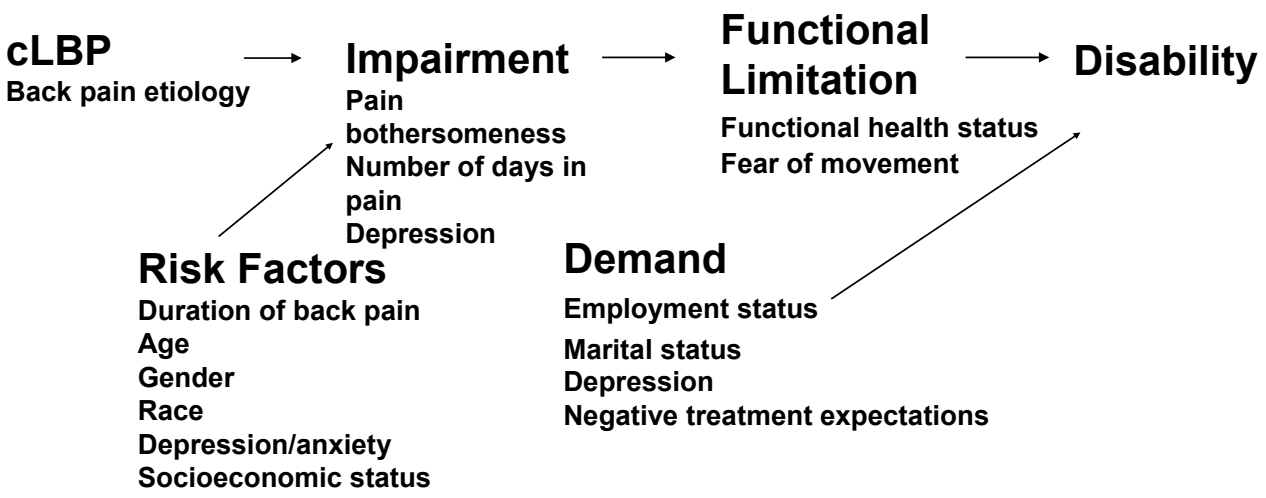

Figure 1.2 Modified Disablement Model with Study Variables and Variable Relationships.

Permission from Elsevier to use Disablement Model; Verbrugge, L. M., \& Jette, A. M. (1994). The disablement process. Social Science and Medicine, 38(1), 1-14. Modified by permission (Alan Jette, personal communication, October 1, 2008). 
burden healthcare providers, insurers and employers. An improved understanding of the patient characteristics associated with improvements in pain and function may reduce the alarming amount of money spent annually for poor treatment outcomes. By examining pre-treatment characteristics of individuals selecting yoga or conventional methods of individualized PT for treatment of chronic low back pain and exploring differences in treatment outcomes this study adds new knowledge useful for healthcare providers and consumers considering the use of yoga for treatment of cLBP.

Preliminary evidence suggests that yoga is a safe and beneficial approach in the long-term management of cLBP. This study adds new knowledge to this preliminary evidence by examining the effects on cLBP of the Integral method of yoga, a different style of yoga than those studied previously by yoga back pain researchers. This study used the same core outcome measures used by previous yoga back researchers to facilitate cross-comparisons of this study's findings to strengthen the evidence of yoga use in the treatment of cLBP and for future yoga systematic reviews and meta-analyses in the treatment of cLBP.

This study contributes to yoga, disability and back pain theory building and testing since the variables chosen in this study were consistent with those recommended for use when evaluating clinical back treatment research. This study tested the usefulness of the Disablement Model (Verbrugge \& Jette, 1994) in designing and evaluating the effects of a yoga back treatment intervention study.

\section{Definitions of Major Concepts}

Following are definitions of key terms:

Chronic low back pain - A recurring pattern of back pain lasting longer than 6-12 weeks that is not associated with an acute injury, fracture, fever, cancer or other disease states such as ureterolithiasis or urinary tract infection (Deyo et al., 1998).

Disability - The impaired ability to perform one's social roles appropriate to age and gender. Disability was measured using the Roland Morris Disability Questionnaire (Roland \& Fairbank, 2000).

Function - The ability to perform activities of daily living without social, emotional or physical impairments. In this study function was measured using the Roland Morris Disability Questionnaire and selected subscales of the RAND SF-36 1.0 (Roland \& Fairbank, 2000; Ware, 2000).

Integral yoga - A method of yoga established by Sri Swami Satchidananda, a monk who dedicated his life to individual and universal peace. The Integral yoga method emphasizes an inclusive meditative approach to classical yoga and includes a specific sequence of yoga postures, progressive relaxation and instruction in the use of the breath (R. Panico \& M. Spears, personal communication, February 21, 2009). 
Kinesiophobia - An irrational and debilitating fear of physical movement and activity resulting from a feeling of vulnerability to painful injury or re-injury (Kori, Miller, \& Todd, 1990). Fear of movement was measured using the Tampa Scale for Kinesiophobia (Vlaeyen, Kole-Snijders, Boeren, \& van Eek, 1995).

MET - Metabolic equivalent, the ratio of a person's working metabolic rate relative to the resting metabolic rate or energy cost of an activity (http://en.wikipedia.org/wiki/Metabolic_equivalent).

Pain bothersomeness - Refers to the amount or degree of pain experienced and will be measured using an 11 point, 0-10, Likert scale (Patrick et al., 1995).

Pain frequency - The number of days per week that pain is experienced.

Pain medication - Includes opioids, non-steroidal anti-inflammatory medications, acetaminophen, herbal products and other medications or substances used specifically for pain relief or control. Pain medication usage was measured in this study by averaging doses used per week and comparing baseline use with that used at 6 and 12 weeks.

Psychological distress - A cognitive, affective and somatic condition that results in depression, lack of well-being and poor psychological functions. Psychological distress was measured using the Beck Depression Inventory v2 (Beck, Brown \& Steer, 1996).

Self-efficacy - A construct described by Bandura (1997) defined as the belief that change can occur through an individual's actions. Self-efficacy was measured using the Back Pain Self-Efficacy Scale, a modification of the Arthritis Self-Efficacy Scale (Levin, Lofland, Cassisi, Porah \& Blonsky, 2003).

Yoga - An empirically derived, formalized system of physical exercises and poses referred to as asanas, breathing exercises referred to as pranayama and relaxation and meditation exercises designed to improve physical, psychological and spiritual wellbeing. Yoga shares philosophical roots with Buddhism and is believed to have originated in India over 5,000 years ago (Miller, 1998).

\section{Assumptions}

Research assumptions for this study were based on the review of literature and the concept relationships included in the Disablement Model. These assumptions are as follows:

1. Individuals with cLBP are able to accurately evaluate their mood, pain characteristics and disability using self-report measures. Previous research supports the use of self-report measures over physical examination findings in 
evaluating clinical outcomes following back pain treatment interventions (Deyo et al., 1998; Deyo \& Weinstein, 2001; Von Korff, Jenson, \& Karoly, 2000).

2. Yoga and physical therapy treatment outcomes may be influenced by individual demographic characteristics including age, gender and socioeconomic status.

3. Treatment outcomes may be influenced by pre-existing pain characteristics, including pain duration, pain frequency, average pain intensity and pain medication usage.

4. Treatment outcomes may be influenced by individual psychological and treatment characteristics such as back pain self-efficacy, depression, fear of movement and treatment expectation and satisfaction.

5. Treatment outcomes may be influenced by minutes of practice per week in a dose response fashion where greater minutes of practice are associated with larger treatment outcome changes.

6. Treatment outcomes may be influenced by use of other treatment modalities not controlled for during the course of the treatment phase of the study.

7. Treatment outcomes may be influenced by previous use of yoga or physical therapy, thereby biasing participant expectations.

8. Chronic back pain exacerbations and remissions cannot be predicted and may occur during the course of this study affecting treatment outcomes. 


\section{CHAPTER 2. REVIEW OF LITERATURE}

This chapter discusses the diagnosis and pathophysiology of chronic low back pain (cLBP). Treatment approaches are discussed and the variety of possible treatments that range from pharmacological basics to cognitive behavior therapy to a wide variety of exercise options. Clinical practice guidelines for treating cLBP and the core domains for evaluating clinical back treatment effects are also included.

\section{Etiology and Diagnosis of cLBP}

Chronic low back pain (cLBP) is defined as back pain that persists beyond the expected time for tissue healing (Ehrlich, 2003), pain persisting for more than 6-12 weeks (Deyo \& Weinstein, 2001) or pain that results in a limitation in usual activity for more than $50 \%$ of the time over a period of 6-12 months (Von Korff et al., 2000). The origin of cLBP can arise from local, referred or systemic causes.

Most cases of back pain result from local biomechanical strain on muscles and connective tissues that resolve spontaneously with conservative treatment (Heffernan, 2001). Biomechanical back pain is thought to arise from inflammation and pressure on pain sensitive structures in the back and spine. These structures include the periosteum of the vertebra, the dura, the facet joints, the annulus fibrosis of the intervertebral discs, epidural veins, longitudinal ligaments and the spinal erector and extensor muscles (Heffernan, 2001; Fields \& Martin, 2001).

Systemic causes of back pain can include infections such as osteomyelitis, inflammatory rheumatic disorders such as ankylosing spondylitis, neoplasms and metabolic diseases such as osteoporosis leading to compression fractures. Back pain can also be referred from abdominal or retroperitoneal processes such as kidney infection, endometriosis and abdominal aortic aneurysm. Identifying a definitive cause of back pain becomes more difficult once these causes of back pain are eliminated and when back pain persists for greater than 6-12 weeks in the absence of obvious trauma or acute fracture (Deyo \& Weinstein, 2001).

The most common causes of cLBP originate from disc disorders and from bony changes in the vertebral column (Heffernan, 2001). Discogenic problems include prolapse or herniation that places pressure on the pain sensitive structures in the back as well as on nerve roots, leading to radicular symptoms such as leg and hip pain. Other causes of cLBP include spinal stenosis, in which the neural foramina become narrowed, causing pressure on nerve roots and resulting in radicular symptoms and pars defects that can cause articulation problems in the facet joints, leading to slippage referred to as spondylolisthesis. Poor posture, physical deconditioning, ligamentous and muscular injuries resulting in spasm and inflammation may lead to cLBP (Heffernan, 2001; Hodges \& Moseley, 2003; Omoigui, 2007). 
Despite advances in diagnostic imaging, researchers have not been able to identify clear associations between diagnostic imaging and physical examination findings with pain symptoms, physical functioning or disability (Deyo \& Weinstein, 2001). For example, $50 \%$ of asymptomatic adults have abnormal radiographic findings similar to symptomatic persons with cLBP, suggesting that imaging studies are of little benefit in evaluating the course of cLBP treatment or treatment outcomes (Fields \& Martin, 2001). Use of diagnostic imaging increases patient costs of care and may not lead to improved treatment selection or successful treatment outcome.

Fewer than $20 \%$ of patients with cLBP have an established etiology for their pain, which makes diagnosis and treatment selection challenging (Heffernan, 2001). Back pain is a subjective experience that does not correlate reliably with abnormal physical examination findings (Heffernan, 2001). Patients with cLBP of unknown etiology represent diverse subgroups whose response to specific treatments is poorly understood (Childs et al., 2004; Keefe, Bradley, \& Crisson, 1990; Riddle, 1998). Additionally, the mechanisms that contribute to the transition from acute to cLBP remain speculative. However, psychosocial factors are known to have a major role (Kovacs et al., 2004).

\section{Pathophysiology of cLBP}

The development of cLBP is complex and begins after an initial injury resulting in tissue damage within the spine, muscles or connective tissues of the back. This damage initiates a cascade of biochemically mediated, neurohormonal changes within tissues and the nervous system resulting in inflammation followed by the subjective awareness and experience of pain (Omoigui, 2007; Pruimboom \& van Dam, 2007).

The release of inflammatory substances following injury stimulates the axons of peripheral nociceptors, pain receptors located in the skin, muscles and connective tissues within the back (Fields \& Martin, 2001). The cell bodies of these peripheral nociceptors are located in the dorsal ganglion of the spinal cord. Stimulation of nociceptor axons activate the release of hydrogen, sodium, potassium and calcium ions from local cells (Hudspith, Siddall, \& Munglani, 2007). These ions activate the secretion of protein peptides including bradykinins, prostaglandins, leukotrines and substance $\mathrm{P}$ from nociceptor endings, which induce an inflammatory response (Hudspith, Siddall, \& Munglani, 2007). Other protein peptides and substances such as serotonin, norepinephrine and nitric oxide are also involved (Fields \& Martin, 2001). Once released, these substances produce a sustained inflammatory response that results in plasticity changes in the nervous system. This response lowers the sensitivity of peripheral nociceptor thresholds, leading to peripheral and central sensitization (Hudspith, Siddall, \& Munglani, 2007).

Peripheral sensitization results in an increased frequency of firing of nociceptors for all stimulus intensities, resulting in hyperalgesia, in which innocuous stimuli produce a sensation of pain (Hudspith, Siddall, \& Munglani, 2007). These impulses are transmitted peripherally to the dorsal root ganglion in the spinal cord and then centrally 
through ascending pathways to regions within the brain and central nervous system (CNS) including the thalamus, amygdala, hypothalamus, limbic system and cerebellum. These CNS regions are part of the pain sensory system involved in the subjective experience and behavioral response to pain (Fields \& Martin, 2001).

Central sensitization is associated with enlargements in the receptive fields of nociceptor cell bodies within the dorsal horn, which alter proprioceptive synaptic pathways resulting in hyperalgesia and disordered proprioceptive reactivity (Fields \& Martin, 2001). These changes are thought to result when inflammatory mechanisms alter biochemical and protein synthesis pathways producing increased neuropeptide synthesis and elevated nitric oxide levels, causing cell damage and circulatory changes within spinal connective tissues and the dorsal horn (Omoigui, 2007).

Cell damage and circulatory changes may contribute to alterations in proprioceptive reflex activity within the spinal column and core spinal stabilizer muscle responses (Hodges \& Moseley, 2003; Radebold, Cholewicki, Polzhofer, \& Greene, 2001). This damage is also speculated to result in changes in muscles and connective tissues, where healthy tissue becomes fibrosed, disturbing motor function and proprioceptive patterns (Langevin \& Sherman, 2007). Loss of normal proprioceptive tone reduces muscle size and strength, thus reducing joint flexibility (Richardson, Jull, Hodges, \& Hides, 1999) and affecting gait, posture and balance, leading to abnormal movement patterns (Hodges \& Richardson, 1999; Langevin \& Sherman, 2007). Abnormal muscular responses and movement patterns lead to pain with movement (Hodges \& Moseley, 2003; Hodges, Pengel, Herbert, \& Gandevia, 2003); Langevin \& Sherman, 2007), restricted activity and physical deconditioning (Pruimboom \& van Dam, 2007) and an increased risk for re-injury (Hodges \& Richardson, 1996).

Atrophy and weakness of the multifidus and lumbar erector spinae muscles involved in spinal segmental support and mobility, have been demonstrated in persons with cLBP by using imaging techniques. (Hodges \& Richardson, 1996; Radebold, Cholewicki, Polzhofer, \& Greene, 2001). Muscle density as well as muscle size has been shown to be decreased in persons with cLBP, compared to normal controls (Richardson et al., 1999), which may contribute to the risk of re-injury and back pain recurrence.

Persons with cLBP have also been found to have delayed contraction onset of the transversus abdominis (TrA) muscle, a band-like muscle which wraps around the abdomen and attaches to the spine along the lumbar and thoracic vertebra (Hodges \& Richardson, 1996). Contraction of this muscle produces a drawing in of the abdominal wall, increasing tension along the spine and providing stability and strength with rotation, flexion and extension movements. Persons with cLBP have been shown to have delayed onset contraction of the TrA when performing movements which destabilize the spine (Hodges \& Richardson, 1996). This is thought to be the result of alterations in the proprioceptive motor control of spinal muscles, which reduces spinal function, causing painful, abnormal movements. 
The mechanisms involved in the transition from acute to chronic pain are speculative and include plasticity changes in the nervous system, genetic variations and expressions and alterations in neuronal sensitivity leading to peripheral and central sensitization (Hudspith, Siddall, \& Munglani, 2007). Once established, cLBP tends to be self-perpetuating due to physical and psychological changes.

The pain experience and the physiological processes associated with pain symptomotology are another speculative area of research that contributes to the challenge in treating individuals with cLBP. The pain sensory system consists of multiple pathways and processes involving physical, biochemical as well as cognitive and emotional factors (Hudspith, Siddall, \& Munglani, 2007). The purpose of the pain sensory system is to protect the body by detecting, localizing and identifying tissue damaging processes. However, chronic pain does not serve a protective or adaptive function. Explanatory models of chronic pain also vary from physiological (Langevin \& Sherman, 2007; Omoigui, 2007) to non-use disease based (Pruimboom \& van Dam, 2007) to biopsychosocial models (Vlaeyen et al., 1995; Weiner, 2008). These models can influence how healthcare providers select treatments and evaluate outcomes contributing to treatment success or failure (Weiner, 2008).

The physical and psychological effects of pain include increased muscular contraction and tension that lead to spasms, increased muscle metabolism, increased plasma cortisol levels, elevated heart rate, increased respiration and blood pressure, increased secretion of inflammatory enzymes and proteins and increased behavioral arousal (Hudspith, Siddall, \& Munglani, 2007). Increased behavioral arousal leads to pain awareness and the emotional response to pain (Fields \& Martin, 2001).

Modulation of pain activation occurs within the dorsal horn through local neuroinhibitory mechanisms and descending pathways from the brain (Hudspith, Siddall, $\&$ Munglani, 2007). These processes activate the sympathetic nervous system, inducing changes in arousal mechanisms activating the stress response. This leads to changes in cardiovascular, respiratory, metabolic and endocrine function (Hudspith, Siddall, \& Munglani, 2007). Modulation through the central nervous system includes secretion of endorphins and enkalephins, which raise pain thresholds, and through cognitive behavioral changes such as fear and avoidance (Vlaeyen et al., 1995).

The emotional consequences associated with cLBP include fear of movement (Grotle et al., 2004; Vlaeyen et al., 1995), depression and anxiety (Deyo \& Weinstein, 2001; Kabat-Zinn, Lipworth, \& Burney, 1985), negative thinking (Buchbinder et al., 2001; Teasdale et al., 2000), and low pain self-efficacy (Altmaier et al., 1993; Asghari \& Nicholas, 2001). These psychological consequences can adversely affect an individual's self-confidence and treatment motivation, which in turn influences the course of cLBP treatment success or failure. A self-perpetuating cycle of fear, dependency and inactivity may result, increasing healthcare utilization and costs of care.

Risk factors for developing cLBP include increased age (Carey, Garrett, Jackman, \& Hadler, 1999), female gender (Marshall \& Murphy, 2008), obesity (Carey et al., 1999), 
prior history of back pain with recurrences (Heffernan, 2001), pain radiating into the leg (Cherkin et al., 1996), restricted spinal range of motion (Thomas et al., 1999), smoking (Carey et al., 1999), poorly localized pain (Wernecke \& Hart, 2001), high levels of pain severity (Carey et al., 1999), psychological distress (Clays et al., 2007), negativity about treatment outcome (Buchbinder, Jolley, \& Wyatt, 2001), pre-existing depression, (Cherkin et al., 1996), low pain self-efficacy (Altmaier, Russell, Kao, Lehmann, \& Weinstein, 1993; Levin, Lofland, Cassisi, Poreh, \& Blonsky, 1996), poor self-rated health (Marshall \& Murphy, 2008), fear of movement (Grotle, Vollestad, Veierod, \& Brox, 2004; Vlaeyen et al., 1995), and minimal daily physical activity (Thomas et al., 1999). Occupational risk factors include jobs involving heavy lifting or sudden maximal efforts with stress on the spine, jobs involving operation of vibratory equipment (Heffernan, 2001) and job dissatisfaction (Thomas et al., 1999). These psychosocial risk factors have been found to be more predictive of cLBP and disability than imaging and physical examination findings and are considered critical factors to include in the assessment of cLBP treatment efforts (Deyo et al., 1998; Marshall \& Murphy, 2008).

\section{Treatment of cLBP}

Treatment approaches for cLBP include conventional medical therapies such as medications, epidural and intra-articular injections and surgery (Deyo \& Weinstein, 2001; Hurwitz, Morgenstern, Kominski, Yu, \& Chiang, 2006), manual therapies including chiropractics (Sherman et al., 2006) and physical therapies including exercise (Hayden, van Tulder, Malmivaara, \& Koes, 2005). Complementary and alternative approaches include yoga, meditation, acupuncture, and massage therapy (Eisenberg et al., 2007; Kabat-Zinn et al., 1985; Quinn, Hughes, \& Baxter, 2006). Cognitive behavioral therapies have also been found to be effective in reducing symptoms (Ostelo et al., 2005). Current evidence-based guidelines indicate that effective treatment of cLBP should address physical as well as psychological components (Chou et al., 2007).

\section{Medical, Chiropractic and Manual Therapies}

Pharmacologic approaches to treating cLBP may be beneficial but involve risks. Long-term use of narcotics and non-steroidal anti-inflammatory medications are associated with serious adverse effects including drug dependence, GI bleeding and nausea (Heffernan, 2001). Narcotic medications may improve mood but do not increase activity levels that are important in improving back function, strength and physical endurance (Deyo \& Weinstein, 2001).

Surgical treatments are rarely helpful because in the absence of progressive neurologic symptoms or cauda equina syndrome, outcomes are inconsistent (Deyo \& Weinstein, 2001). Additionally, between 5\% and 15\% of these surgeries result in poor outcomes requiring repeated surgery (Heffernan, 2001). Evidence supporting epidural corticosteroid injections is also inconsistent (Heffernan, 2001). 
Systematic reviews evaluating effects of alternative therapies, including yoga, acupuncture, spinal manipulation and massage therapies, have shown them to be beneficial (Kalauokalani, Cherkin, Sherman, Koepsell, \& Deyo, 2001; Sherman et al., 2006; van Tulder, Furlan, \& Gagnier, 2005). However, when patients lack insurance coverage for alternative therapy, costs of care may be substantially greater (Eisenberg et al., 2007), and it is not clear whether the additional costs lead to improved long-term outcomes (Lind et al., 2005).

\section{Cognitive Behavioral Therapies}

Cognitive behavioral therapy (CBT) has proven beneficial in the treatment of cLBP, as reported in a number of systematic reviews and meta-analyses (Ostelo et al., 2005; Turk, 2002; van Tulder et al., 2000). CBT approaches involve teaching individuals self-regulatory behaviors using cognitive reappraisal techniques to modify beliefs and attitudes. Cognitive reappraisal techniques enable individuals to modify and "reframe" negative thinking patterns associated with pain and fear, which reduces the stress response and promotes improved functioning (Kabat-Zinn, Lipworth, \& Burney, 1985; Langer, 1989). These methods are associated with improved pain self-management and greater acceptance of situational experiences, thus reducing adverse effects of the pain experience (Kabat-Zinn, 1982). Other reported benefits include increased self-esteem and self-efficacy (Bandura, 2001; Waelde, Thompson, \& Gallagher-Thompson, 2004) and reduced emotional distress and pain (Zautra, Johnson, \& Davis, 2005).

Progressive relaxation and mindfulness-based meditation are two specific CBT techniques found effective in treating cLBP (Benson, 2000; Carson et al., 2005; KabatZinn et al., 1985; Lau \& McMain, 2005). Progressive relaxation teaches the individual awareness of the physical sensations of tension and relaxation in muscle groups so as to counteract sensations associated with the stress response (Benson, 2000; Esch,

Fricchione, \& Stefano, 2003). Progressive relaxation has been found effective in reducing cLBP symptom intensity (Ostelo et al., 2005).

Mindfulness-based meditation teaches intentional self-regulation of attention to physical sensations. This allows the individual to detach from distressing sensations and thoughts when experiencing discomfort, thereby reducing pain symptoms (Kabat-Zinn et al., 1985), depression and anxiety (Kabat-Zinn et al., 1985; Teasdale et al., 2000) and improving self-acceptance (Baer, 2003; Kabat-Zinn, 1982; McCracken, Vowles, \& Eccleston, 2005). Cognitive behavioral benefits have been shown to persist over time, suggesting these methods are self-reinforcing and promote treatment adherence (Miller, Fletcher, \& Kabat-Zinn, 1995).

Research exploring physiological mechanisms associated with cognitive behavioral approaches have found decreased catecholamine levels to be associated with improvements in self-efficacy (Bandura et al., 1985) and changes in cortisol levels associated with improvements in depression (Carlson, Speca, Patel, \& Goodey, 2004; Robert-McComb, Tacon, Randolph, \& Caldera, 2004). These biochemical changes are 
thought to modulate adverse biochemical and cellular effects associated with chronic pain, reducing SNS reactivity, inflammatory biochemical levels and muscle tension (Esch et al., 2003; Esch, Fricchione, Stefano, \& Benson, 2002). A disadvantage of CBT is that the methods are not as effective in improving physical conditioning and spinal functioning when compared to exercise-based treatments (Ostelo et al., 2005; van Tulder et al., 2000).

\section{Exercise and Exercise-Based Therapies}

Two meta-analyses evaluating over 95 high quality randomized controlled trials (RCTs) concluded that exercise therapy is effective in decreasing pain, reducing absenteeism and improving function in persons with cLBP (Hayden et al., 2005; Liddle, Baxter, \& Gracey, 2004). Other benefits include physical conditioning, increased endurance (Deyo \& Weinstein, 2001; Heffernan, 2001) and improved mood (Phillips, Kiernan, \& King, 2003; Turk \& Monarch, 2002; Turk \& Okifuji, 2002). However, the benefits from exercise have not been shown to decrease the incidence or duration of cLBP recurrences (Lahad, Malter, Berg, \& Deyo, 1994). Although exercise is associated with persistent, long-term improvements in back pain and disability (Hurwitz, Morgenstern, \& Chiao, 2005; Mortimer, Pernold, \& Wiktorin, 2006), long-term outcomes are strongly influenced by treatment adherence and patient motivation (Liddle et al., 2004).

Most back exercise treatment programs include components for strengthening spinal erectors and core abdominal and torso muscles to reverse paraspinal muscle atrophy and loss of proprioceptive tone (Vad, Bhat, \& Tarabichi, 2007). Atrophy and weakness of the multifidus and lumbar erector spinae muscles involved in spinal segmental support and mobility have been demonstrated in persons with cLBP using imaging techniques (Hodges \& Richardson, 1996; Radebold, Cholewicki, Polzhofer, \& Greene, 2001). Exercises that isolate and strengthen core spinal stabilizers and increase kinesthetic awareness of the spine and spinal stabilizer muscles improve back function and reduce the risk of pain exacerbation and re-injury (Lehman, 2004; McDonald \& Lundgren, 1998; Richardson et al., 1999).

Hurwitz et al. (2005), comparing the long-term effects of intensive exercise-based rehabilitation programs on cLBP symptoms and function with those resulting from recreational exercise, found greater improvements in depression and disability resulting from participation in recreational exercise. The authors proposed that the improved outcomes from recreational exercise may be due to the added effects of group support and interaction. Another possibility is that recreational exercise involves physical activity performed for pleasure rather than for treatment, and engaging in pleasurable activities, despite discomfort, is thought to be a key factor in the success of acceptance-based back treatment programs such as mindfulness-based meditation interventions (McCracken, Vowles, \& Eccleston, 2005). 


\section{Multidisciplinary Programs}

Multidisciplinary programs provide the benefits of medical therapies in addition to those of exercise and CBT. These programs have proven to be effective in reducing pain and improving function in persons with cLBP through combined physiological and psychological effects (Guzman et al., 2002; Patrick et al., 2004). However, treatment outcomes vary, depending on what therapeutic components are included or emphasized within the program (Guzman et al., 2002; Patrick et al., 2004). Multidisciplinary programs are also costly and time intensive, which influences treatment adherence and treatment outcomes (Guzman et al., 2002). Recent studies have also failed to demonstrate superior long-term benefits resulting from intensive approaches, thus suggesting that they are not cost effective, which is a significant economic concern (van Geen, Edelaar, Janssen, \& van Eijk, 2007).

\section{Yoga}

The use of yoga for achieving health and treating illness is centuries old and emerged from India. The earliest texts that describe yoga science and theory are attributed to Patanjali and are estimated to have been recorded around the third century A.D. (Miller, 1998). According to Sanskrit scholars, Patanjali is credited with systematizing the philosophy and practice of yoga from his own study of the ancient Hindu Vedas (Feuerstein, 1989; Hartranft, 2003).

The key concepts and formalized principles of yoga were recorded and passed down through oral tradition by Patanjali and his students in phrases of Sanskrit prose known as the Yoga-Sutras, which are still studied by yoga practitioners worldwide (Feuerstein, 1989). The sutras describe yoga purposes, principles and practices and the benefits that result from the daily study and practice of yoga (Satchidananda, 1990). In Sutra 1.2 Patanjali describes the purpose of yoga as the restraint or cessation of the turnings of thought (Miller, 1998; Sachidananda, 1990).

Yoga is not a religion but rather a science of consciousness and system for enhancing human development based on physical, cognitive and spiritual practices (Iyengar, 1976; R. Panico \& M. Spears, personal communication, November 6, 2008). The origin of the word yoga is Sanskrit and means "to yoke," join or unite (Feuerstein, 2001). According to classical yoga philosophy, the goal of yoga is control of the mind, body and senses through disciplined practices (Miller, 1998). The practices of yoga achieve this goal through methods that condition the physical body and improve mental concentration (Miller, 1998). These practices lead to mental tranquility and spiritual freedom; "if you can control the rising of the mind into ripples then you have achieved yoga" (Sanchidananda, 1990, p.4).

Yoga is described as both a process as well as a state of being - a discipline and its goal (Miller, 1998). "Yoga is the method by which the restless mind is calmed and the energy is directed into constructive channels," leading to physical and emotional well- 
being (Iyengar, 1979, p. 20). Control of the mind and senses results from the gradual mastery of sense-withdrawl a state of consciousness referred to as pratyahara. Pratyahara is an interior process of mindfulness in which sensory perceptual events in the mind become reconfigured (R. Panico, personal communication, January 25, 2009). Sutra 1.3 describes it this way: "When thought ceases, the spirit stands in its true identity as observer to the world." Pratyahara enables the sensory perceptual events of the exterior or "material mind" to become transformed through a process of interiorization (R. Panico, personal communication, January 25, 2009). This process allows the material mind to join with "pure consciousness" leading to spiritual liberation and physical well-being (Rao, 2005).

Yoga is classically researched through direct experience by the yoga practitioner under the guidance of the yoga teacher or master. The teacher, or guru, explains yoga concepts, principles and practices guiding and framing the student's physical and psychological experiences within the theoretical framework of Patanjali's precepts (Iyengar, 1976; Rao, 1998). This direct experiential, phenomenological approach is the process by which classical yoga science and theory has been validated for centuries (Feuerstein, 1989).

Classical yoga consists of eight aspects referred to as the eight limbs of Astanga yoga. These limbs build upon each other, incorporating three levels of experience. The first level deals with the moral and ethical precepts or exterior yoga practices of behavior and attitudes. The second level deals with the physical practices directed toward the body, breath and sensory events. These practices include physical exercises and poses referred to as asanas and controlled breathing methods referred to as pranayama. The third level deals with the inner practices which includes concentration, sense-withdrawal, deep relaxation and meditation practices that lead to the superconscious states referred to as samadhi (Feuerstein, 2001; R. Panico, personal communication, January 25, 2009). Activation of superconscious states results from mastery and disciplined adherence to each level of experiential practice.

The purpose of asana practice is to condition the body, thereby increasing strength, flexibility and endurance. Practicing the poses also improves concentration, focus and awareness of body sensations (Satchidananda, 1990). Yoga postures stimulate body organs, promoting digestion, improving circulation and nervous system functioning (Iyengar, 2005). According to classical yoga theory, the asana practice prepares the body to sit for prolonged periods of meditation necessary to gain mastery of thoughts and senses (Feuerstein, 1989).

Pranayama methods enhance mental concentration, calm the nervous system and direct the circulation of prana in the body (Iyengar, 2005). Prana is considered a vital energy or life force which circulates within the body and, if blocked or altered, is thought to result in physical and mental illness (M. Spears, personal communication, November $6,2008)$. In Western terms, prana encompasses vital processes at cellular and biochemical levels. Pranayama exercises condition the body through controlled activation of the sympathetic and parasympathetic nervous systems, altering conditioned nervous 
system responses (Iyengar, 1976). The control of breathing patterns through pranayama also improves mental focus and concentration necessary for meditation (Satchidananda, 1990).

Like pranayama, the meditation and relaxation components of yoga (yoga nidra) activate the parasympathetic nervous system. This is thought to modulate the stress response and sympathetic nervous system overactivity (Benson, 2000; Kabat-Zinn, 1982). Meditation practice promotes "detached awareness," sense-withdrawl and pratyahara. These processes promote a shift from conditioned reactivity and propagation of secondary emotions arising from external sensory and perceptual events leading to a state of greater mindfulness (R. Panico, personal communication, January 25, 2009). This enhanced state of mindfulness and detached awareness is associated with improvements in mood and pain reactivity (Kabat-Zinn et al., 1985; Teasdale et al., 2000).

Many different styles of yoga currently are practiced in the U.S., each having a unique focus and emphasis. The styles of yoga reportedly found beneficial in treating cLBP include Viniyoga (Sherman et al., 2005), Iyengar yoga (Williams et al., 2005), hatha yoga (Galantino et al., 2004; Uyterhoeven, Khalsa, \& Whittemore, 2008) and Integral yoga (Dowling, Carter \& Panico, 2008; Michels et al., 2006). Although each of these styles emphasize the therapeutic use of yoga in restoration of function and stress reduction, the methods differ in use of props, sequencing of poses and incorporation of relaxation, meditation and breathing components.

In 2005 two NIH-funded RCTs examining the effects of yoga on cLBP found yoga to be superior to conventional back exercise or back education classes in reducing disability, pain and pain medication use (Sherman et al., 2005; Williams et al., 2005). Yoga benefits also persisted over time, including a reduced need for pain medication use, which suggests that yoga can reduce the iatrogenic risks associated with pharmacologic therapies (Sherman et al., 2005).

Studies evaluating the direct effects of yoga on the musculoskeletal system have reported that changes within the architecture of the spine and pelvis begin within 8 weeks of group instruction (DiBenedetto et al., 2005; Greendale et al., 2002). Greendale et al. investigated the effects of Iyengar yoga in 21 women with osteoporosis and hyperkyphosis and found improvements in physical performance measures including upper extremity reach and increased spinal height measurements after 12 weeks of group instruction. DiBenedetto et al. examined the effects of an 8-week Iyengar yoga program in 23 elderly women and found changes in hip extension and stride length that were associated with improvements in gait. Galantino et al. (2004) found improved spinal flexibility measures in 22 adults following a 6-week hatha yoga intervention with significant improvements in mood and disability compared to a no treatment control group. Yoga has also been shown to improve balance (Bastille \& Gill-Body, 2002; Galantino et al., 2004; Gauchard, 1999) and lower extremity muscle strength (Brochu, 2002), indicative of improved proprioceptive tone and function. 
Several proposed mechanisms are used to explain the neuromuscular benefits of yoga. One idea is that the repetitive stretching and force resistance movements of yoga postures increase circulation to muscles and connective tissues (Riley, 2004). This increase lowers inflammatory substance levels, reducing pain and the risk of progressive nervous system and connective tissue damage (Langevin \& Sherman, 2006).

Yoga postures also require coactivation of primary and antagonist muscles groups during flexion and extension movements. This stimulates intrafusal and golgi tendonorgan feedback mechanisms, improving proprioceptive tone (Riley, 2004) evidenced by increased muscle size, strength and endurance (Brochu, 2002) and the return of normal joint range of motion and flexibility (Galantino et al., 2004). These changes can reverse abnormal movement patterns resulting from pain and muscle atrophy, thereby reducing the risk of back re-injury (Langevin \& Sherman, 2006). Improved proprioceptive tone and spinal stability also results from the emphasis on proper skeletal alignment during performance of yoga poses that strengthens core abdominal and spinal stabilizer muscles, further reducing the risk of injury and painful movement.

Studies evaluating yoga effects on the cardiovascular system have found that yoga improves cardiovascular conditioning (Harinath et al., 2004; McCaffrey, Ruknui, Hatthakit, \& Kasetsomboon, 2005; Murugesan, Govindarajulu, \& Bera, 2000), an important component in improving the health status of persons with cLBP. Harinath, et al., (2004) conducted an RCT comparing yoga to an exercise conditioning program. After 3 months of treatment, yoga use produced significantly greater improvements in endurance and pulmonary function and was associated with greater reductions in heart rate and blood pressure. The hemodynamic and metabolic demands of yoga have been found to range from a MET energy cost of 6.7-similar to walking and dance, if done vigorously (Carroll, Blansit, Otto, \& Wygand, 2003) - to 2.17 METs when performed less vigorously using props (Clay, Lloyd, Walker, Sharp, \& Pankey, 2005).

Yoga effects on the nervous system have led to other proposed mechanisms of action. Theses effects include improved baroreceptor tone and orthostatic tolerance (Harinath et al., 2004; Selvamurthy et al., 1998), increased heart rate variability (Shannahoff-Khalsa, Sramek, Kennel, \& Jamieson, 2004) and reduced levels of cortisol and catecholamines (Benson, 2000; Harinath et al., 2004; Michalsen et al., 2005; Minvaleev, Nozdrachev, Kir'yanova, \& Ivanov, 2004; Selvamurthy et al., 1998). Yoga results in reduced serum catecholamine and cortisol levels, increased endorphin levels and changes in pituitary hormone levels (Benson, 2000; Brown \& Gerbarg, 2005; Michalsen et al., 2005). These changes are thought to result from the controlled breathing practices, deep relaxation and meditative components of yoga (Benson, 2000; Iyengar, 1976; Kabat-Zinn, 1982). The subjective sense of calm, improved mood and reduced stress (Benson, 2000; Brown \& Gerbarg, 2005) from yoga may also be due to increased serum melatonin levels found following yoga use (Minvaleev, Nozdrachev, Kir'yanova, \& Ivanov, 2004).

Numerous studies have found that yoga reduces anxiety and depression (KabatZinn et al., 1985; Lavey et al., 2005; Lee, Mancuso, \& Charlson, 2004; Michels, 
Edwards, Salstrom, Spears, \& Panico, 2006; Waelde, Thompson, \& GallagherThompson, 2004; Woolery, Myers, Sternlieb, \& Zeltzer, 2004), which are known to interfere with back pain treatment outcomes (Deyo \& Weinstein, 2001). These effects may be another mechanism for explaining improvements in back pain and function resulting from yoga.

Another mechanism may be the improved ability to change physiologic reactions to distressing sensations that leads to reduced anxiety and pain observed following yoga use (Esch et al., 2003; Kabat-Zinn et al., 1985). Control of physiologic reactivity, cognitive reactivity and sensory awareness is the purpose of yoga according to the ancient Yoga-Sutras (Desikachar, 1999; Miller, 1998). Control of sensory awareness and reactivity leads to improved physical and psychological functioning (Feuerstein, 1989; Satchidananda, 1990) and may explain why use of yoga promotes greater improvements in pain and function when compared to other conventional treatment approaches for cLBP.

\section{Core Domains and Clinical Practice Guidelines for Treatment of cLBP}

In May 1997 a group of international back pain researchers met to establish a standardized set of core outcome measures for use in conducting clinical back pain research to improve comparisons of back treatment outcomes (Deyo et al., 1998). Based on clinical and scientific evidence, the researchers recommended the use of self-report measures when evaluating treatment outcomes in research and clinical settings.

Self-report instruments are more valid in assessing changes in function and are more closely associated with readiness to return to work than are direct measures of physical function, diagnostic imaging or physical examination findings (Deyo et al., 1998; Ferguson, Marras, \& Gupta, 2000; Fishbain, 2003). The Agency for Health Research and Quality (AHRQ) (http://www.ahrq.gov) and the American College of Physicians and the American Pain Society also support use of self-report measures in evaluating treatment outcomes in clinical practice (Chou et al., 2007).

The 1997 recommendations also established six core outcome domains necessary for evaluating the effectiveness of clinical back treatment methods. The core research domains and recommended methods for evaluating back treatment outcomes are summarized as follows:

- Pain: Symptom bothersomeness using a Likert or visual analog scale.

- Function: SF-36

- Well-being: SF-36

- Disability: Roland-Morris or Oswestry Questionnaire

- Disability social role: During the past 4 weeks, how any days did your pain prevent you from going to work or school?

- Satisfaction with care: Over the course of treatment for your low back pain, how would you rate your overall medical care? 
Satisfaction with care is considered a broad construct that can be measured by assessing treatment expectations, perceptions of provider performance and treatment efficacy (Evans et al., 2004). Satisfaction with care is an important construct, since it is associated with treatment adherence and improved treatment outcomes.

Self-efficacy beliefs, depression and fear of movement are also considered important when assessing back treatment outcomes. According to Bandura (1997), selfefficacy beliefs are modifiable and create the foundation for self-regulation and control of automatic responses to pain and negative thinking. Self-efficacy beliefs improve the incentive to persevere to achieve goals important in back treatment adherence.

High self-efficacy is positively associated with increased activity levels, improved back function and improved pain outcomes in individuals with cLBP (Altmaier et al., 1993; Asghari \& Nicholas, 2001; Lackner \& Carosella, 1999; Levine, 1996). High levels of self-efficacy are also positively associated with persistent long-term improvements in pain and function (Asghari \& Nicholas, 2001).

Fear of movement is important when assessing back treatment outcomes because high fear of movement can adversely impact activity levels, treatment adherence and back treatment success. Lower fear of movement levels are associated with increased activity and improved mood and function in individuals with cLBP (Grotle et al., 2004; Vlaeyen et al., 1995).

Current clinical practice guidelines recommend assessing for depression when patients with cLBP fail to improve after 6 weeks of treatment (Chou et al., 2007). Depression is known to interfere with treatment motivation and to adversely impact back treatment outcomes (Cherkin et al., 1996; Deyo \& Weinstein, 2001).

\section{Summary and Conceptual Framework}

This review of literature has found a variety of approaches effective in the treatment of cLBP, including pharmacological agents, exercise, cognitive behavior therapies and yoga. The results also indicate that predicting treatment outcomes based on patient characteristics is not clear, which suggests the need for further research. Based on these conclusions, this study was designed to explore the clinical and demographic characteristics and core outcomes of individuals self-selecting yoga versus conventional physical therapy care for treatment of cLBP. A goal of the study is to improve healthcare provider knowledge in understanding factors associated with improved back treatment outcomes. The selection of variables used in this study is based on this review of literature. Associations between variables are based on the Disablement Model, which is used as the conceptual framework in this study (Verbrugge \& Jette, 1994). The Disablement Model describes the process and factors that influence cLBP function or disability. Since the primary goal in treating cLBP is to improve function and reduce disability, the Disablement Model was chosen to describe and interpret this study's 
findings. Figure 1.2 depicts the Disablement Model with modifications that include study variables. 


\section{CHAPTER 3. METHODS}

This chapter discusses the research design, instruments and procedures used to examine and compare persons selecting yoga versus physical therapy for treatment of cLBP. Descriptions of the samples, settings, interventions and procedures for data collection, statistical analysis and protection of human subjects are included.

\section{Research Design}

This descriptive study used a prospective, quasi-experimental design to describe and compare two convenience samples of individuals with cLBP. The study was conducted in Athens, Georgia, from January to November 2008. Yoga volunteers ( $\mathrm{n}=$ 27) were recruited from the Athens Regional Medical Center's Mind-Body Institute, which offers on-going 6 week, once a week, 2 hour modified Integral yoga classes for individuals with cLBP. Physical therapy volunteers $(n=26)$ were recruited from the Athens Regional Medical Center Rehabilitation Department and the Physician's Back and Neck Clinic, a private out-patient physical therapy clinic.

Participants were compared on demographic, psychological, treatment, and clinical characteristics at baseline, 6 and 12 weeks. Verbal and written consent was obtained at enrollment for yoga participants and at intake for PT participants. Baseline questionnaires were either mailed to participants or provided to them in the clinic settings prior to the first PT or yoga class session. Post-treatment questionnaires were administered at 6 weeks following the conclusion of the last yoga class for yoga participants or at the conclusion of 6 weeks of once to twice weekly physical therapy sessions for PT participants.

A total of 21 yoga and 21 PT participants completed the 6 week assessments. A $\$ 10.00$ incentive was provided to participants who completed the 6 week assessment. A third assessment was conducted at 12 weeks. A total of 21 yoga and 16 PT participants completed the 12 week assessment. Five PT participants were unable to be located to complete the 12 week assessment questionnaire. Figure 3.1 shows the study design and reasons for attrition.

\section{Sample, Setting and Recruitment Procedures}

A convenience sample of 53 adult volunteers meeting eligibility criteria were recruited for this study. Participants were considered eligible for the study if they were adults over age 18 , reporting a history of back pain with recurrences for a minimum of at least 12 weeks meeting the following inclusion/exclusion criteria: 

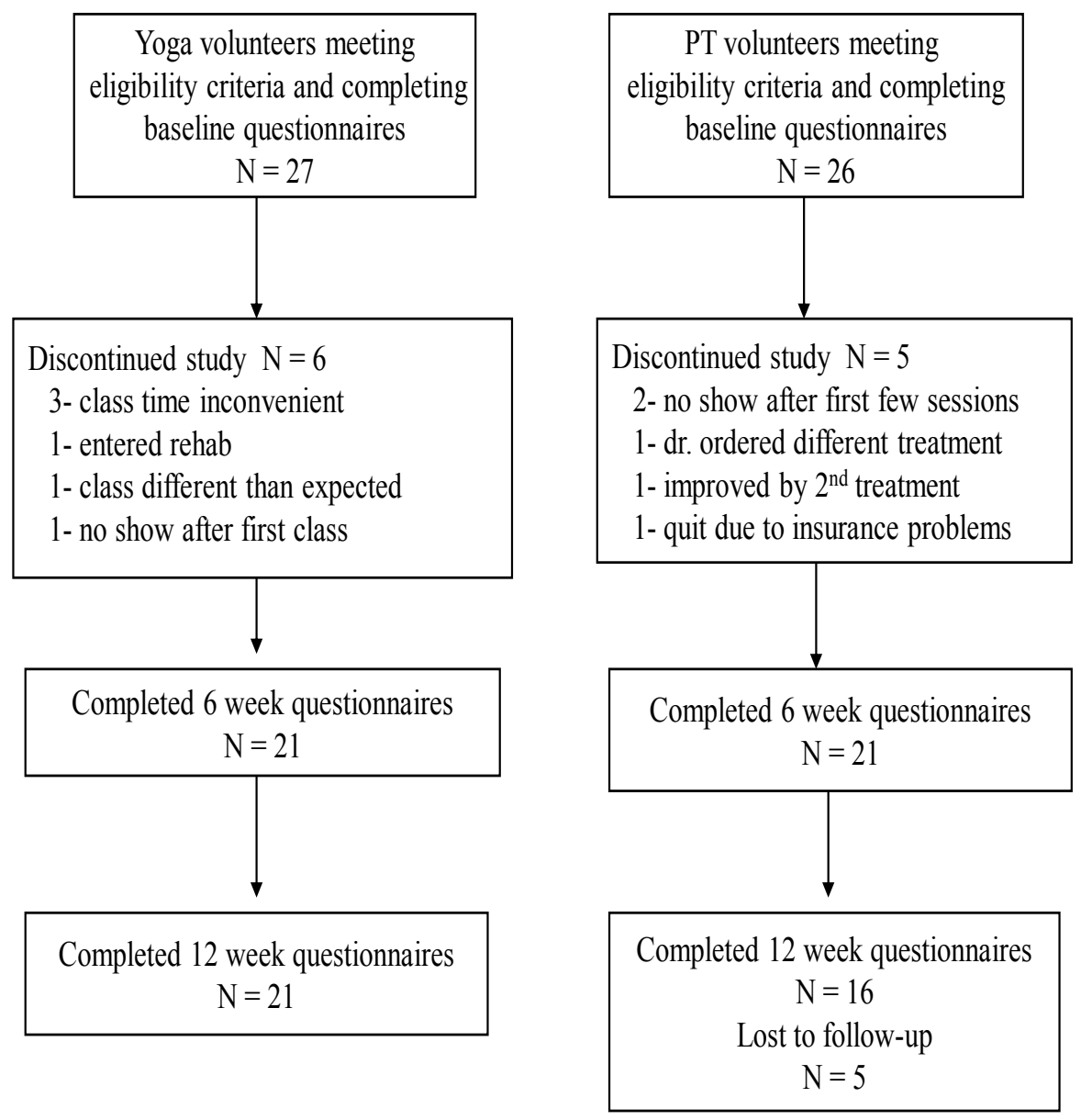

Figure 3.1 Study Design. 


\section{Inclusion Criteria}

- Presence of intermittent or continuous low back pain for at least 12 weeks based on self-report or physician referral.

- Enrollment into physical therapy or yoga for treatment of cLBP.

- Ability to read and speak English.

\section{Exclusion Criteria}

- Persons under 18 years of age (since cLBP is not a common medical problem in children).

- Persons with serious health co-morbidities such as cancer or heart disease.

- Persons whose back pain was related to acute fracture, severe neuropathy or nonmusculoskeletal etiologies.

Yoga participants $(n=27)$ were recruited from five separate six-week yoga class series conducted at the Athens Regional Medical Center's Mind Body Institute. The clinic receptionist informed yoga participants about the study during class enrollment periods. Participants who were interested were then contacted by the investigator, who obtained consent, screened them for eligibility and answered questions about the study. Baseline questionnaires, including the Survey Consent Form (Appendix A), were then mailed to study participants for completion prior to the start of the first yoga class session. The investigator also was present at the first yoga class to meet participants and to administer and collect the baseline questionnaires.

Physical therapy participants $(n=26)$ were recruited from two clinical settings: the Physician's Back and Neck Clinic and the Athens Regional Medical Center Rehabilitation Department. PT participants recruited at the Physician's Back and Neck Clinic were informed about the study if they met inclusion criteria by the nurse practitioner or physician during the initial medical screening appointment prior to beginning their first physical therapy session. If interested, participants were given the Survey Consent Form to read and the baseline questionnaires to complete and return to their physical therapist at the first physical therapy session. The investigator's phone number was provided to all participants. The investigator called each participant to answer any questions about the study and to verify eligibility criteria and consent.

At the Physician's Back and Neck Clinic, individual physical therapists were also involved in recruiting participants who met eligibility criteria if they had not been informed previously by the physician or nurse practitioner. In these cases, participants were informed about the study during their PT assessment appointment. If agreeing to participate, they were given the survey consent form and the baseline questionnaires to complete and return at their next appointment. A total of 17 participants were recruited from this site.

The clinic receptionist at the Athens Regional Medical Center Rehabilitation Department helped to recruit participants by informing them about the study when calling 
to schedule their initial physical therapy appointment. If they were interested, the investigator then contacted them by phone to determine eligibility, describe study details and obtain verbal consent to participate. Baseline questionnaires, including the Survey Consent Form, were then mailed for completion to be returned at the first physical therapy evaluation session.

Individual physical therapists at the Athens Regional Medical Center Rehabilitation Department also recruited eligible participants who met inclusion criteria if they had not been informed previously informed by the receptionist prior to the physical therapy evaluation session. In these cases, the physical therapist informed eligible participants about the study, provided them with the survey consent form and baseline questionnaires. If they were interested, the investigator was then notified so that participants could be contacted to discuss study details and obtain consent. A total of 9 participants were recruited from this site.

\section{Sample Size Calculations}

Sample size calculations were estimated using PASS power calculation software (PASS 2002) to test the null hypothesis at $\alpha=.05$ and $\beta=.20$. Sample calculations were based on pre-post change score sample means and standard deviations obtained by Sherman et al., (2005) using the Roland Morris Disability Questionnaire (RMDQ) to measure yoga treatment effects. Other research using the RMDQ as the criterion for measuring back treatment effects suggests that a pre-post change score of 2-3 represents clinically meaningful change and avoids under-powering a study when used for sample size calculations (Beurskens, de Vet, \& Koke, 1996; Roland \& Fairbank, 2000). The PASS power analysis estimated that a sample size ranging from 9 to 32 for each group was sufficient to obtain a power of .80 to detect a moderate effect size (Cohen, 1977) at a significance level of .05 based on a t-test analysis of group differences. To allow for attrition, group samples sizes of at least 20 were recruited.

\section{Concepts, Variables and Instruments}

The selection of study concepts, variables and instruments was based on the review of the yoga and back pain research literature. Study instruments were selected to adhere to the standardized core back research outcomes recommended by Deyo (1998) and those used in previous cLBP yoga research to facilitate cross-comparison of findings.

\section{Background and Demographic Characteristics}

A Baseline Demographic Questionnaire, included in Appendix B, was developed to assess group differences and similarities in age, gender, race, income status, marital status, work status, educational level and duration and etiology of cLBP. Questions regarding treatment expectations and anticipated benefits were also included. 


\section{Disability}

The Roland Morris Disability Questionnaire (RMDQ), a 24-item, selfadministered questionnaire, was used to assess physical function and disability affected by low back pain (Appendix C). The RMDQ was developed for use in clinical and research settings and is offered free for public use (Roland \& Fairbank, 2000). Scores on the RMDQ range from $0=$ no disability to $24=$ maximum disability. Total scores are calculated by assigning a " 1 " to each item checked and a " 0 " to each unmarked item. The instrument takes 5 minutes to complete and has a readability grade level of 5.6.

The RMDQ was validated in a primary care population of low back pain patients, and normative data for improved and unimproved patients with cLBP are available (Roland \& Fairbank, 2000). The RMDQ has shown responsiveness in detecting clinically meaningful change following treatment (Roland \& Fairbank, 2000). Validity and reliability coefficients range between .84-.93 (Roland \& Fairbank, 2000;. Roland, 1983).

Studies evaluating clinically significant and meaningful changes in activity and function have determined that change scores of 2.5 to 5 points are significant in discriminating between improved and unimproved patients scoring in the moderate to high levels of disability (scores $\geq 8$ ) after 5 weeks of treatment (Beurskens et al., 1996). Stratford, Binkley, Riddle \& Guyatt (1998) found that a 1 to 2 point change score can be clinically important in less disabled populations (RMDQ scores $\leq 5$ ).

Sherman et al., (2005) reported both statistically and clinically significant improvements in disability with pre-post change scores of 2.5 on the RMDQ following an 8 week Viniyoga intervention. Similar changes were also reported by Uyterhoeven, Khalsa, \& Whittemore (2008) in a study of the effects of hatha yoga on cLBP. Change scores of 2 to 3 points on the RMDQ are recommended for sample size calculations (Roland \& Fairbank, 2000).

\section{Depression}

The Beck Depression Inventory-II (BDI-II) was used to measure mood and depressive symptomotology (Beck, Brown, \& Steer, 1996). The BDI-II is a widely used, 21-item, self-administered, self-report questionnaire that takes approximately 5 to 10 minutes to complete and requires a $6^{\text {th }}$ grade reading level. Each item is scored from 0 to 3 , with higher summed scores representing greater depressive symptoms. Scores range from 0-63 where scores of 0-13 are considered normal, scores of 14-19 represent mild to moderate depressive symptoms, scores of 20-28 represent moderate to severe depressive symptoms, and scores of 29-63 represent severe depressive symptoms.

The BDI-II has shown high internal consistency and reliability in an outpatient sample (coefficient $\alpha=$.92) (Beck et al., 1996). Content validity was established using DSM-III categories for diagnosis of depression (Groth-Marnat, 1990). Concurrent validity has been established by correlating the BDI-II with other scales measuring 
depression, including the Hamilton Psychiatric Rating Scale (Pearson's $r=.73$ ) and the MMPI Depression Scale (Pearson's $r=.76$ ) (Groth-Marnet,1990). The BDI-II was used and found to be reliable by Galantino (2004) when evaluating the effects of yoga on depression in individuals with cLBP.

\section{Health Status}

The RAND SF-36 1.0 (http://www.rand.org) (Appendix D) was used to assess health status. The RAND SF-36 1.0 is free for public use and is one of the recommended core outcome measures for use in clinical back treatment research (Deyo et al., 1998).

The RAND SF-36 1.0 is a self-administered, 36-item, self-report questionnaire that takes 7-10 minutes to complete and requires a $7^{\text {th }}$ grade reading level. The instrument was designed for both research and clinical applications (Ware, 2000), is one of the most widely used instruments for assessing health status and functional changes in adults with musculoskeletal disorders (Beaton, Hogg-Johnson, \& Bombardier, 1997) and has been used in previous yoga back pain research (Sherman et al., 2005).

The questionnaire contains a variety of response options, including yes/no answers and items scored on a 1-5 rating scale. Each item is scored from 0 to 100, and all items are scored so that higher scores indicate a more favorable health status. Pre-coded numeric values and reverse coding instructions for some items are included in the scoring key. Individual items are aggregated into eight subscales measuring eight health concepts: physical functioning, role limitations due to physical health problems, role limitations due to personal or emotional problems, energy/fatigue, emotional well-being, social functioning, bodily pain and general health perceptions. Subscale scores are obtained by averaging individual item scores.

Normative data for the RAND SF-36 1.0 has been established in a variety of subset populations of patients with cLBP (Pahl et al., 2006; Von Korff, Jenson, \& Karoly, 2000; Ware, 2000). Content and concurrent validity were established through correlations showing that lower subscale scores are associated with unemployment and increased pain severity (Von Korff et al., 2000). Reliability of the bodily pain subscale ranges between 0.79-0.96 (Ware, 1993).

The RAND SF-36 1.0 has shown responsiveness in detecting improvements at repeated intervals in patients with cLBP who rate themselves improved discriminating between patients who improved from those who did not (Taylor, Taylor, Foy, \& Fogg, 1999). Although all the RAND SF-36 1.0 subscales have been found to be responsive to change in cLBP populations, the physical function, social function and bodily pain subscales are the most highly responsive to change following clinical interventions and are recommended for use in clinical back treatment research (Taylor, 1999). Change scores ranging from 3-7 points are considered clinically significant (Deyo et al., 1998; Stewart, Maher, Refshauge, Bogduk, \& Nicholas, 2007).

\section{Self-Efficacy}


Self-efficacy was assessed using the Back Pain Self-Efficacy Scale (BPSES ), an adapted version of the Arthritis Self-Efficacy Scale (Levin et al., 1996) and Chronic Pain Self-Efficacy Scale (Anderson, Dowds, Pelletz, Edwards, \& Peeters-Asdourian, 1995). According to Bandura (1997) self-efficacy instruments should be tailored to the construct or domain of interest. The BPSES measures a person's perceived capacity to cope with back pain symptoms (Levin et al., 2003).

The BPSES is a self-administered, 20-item, self-report instrument containing three subscales; self-efficacy for pain management, self-efficacy for functional ability and self-efficacy for controlling symptoms (Appendix E). The instrument is free for public use and requires an $8^{\text {th }}$ grade reading level. Questionnaire items are rated on a 10point Likert scale, ranging from $10=$ very uncertain to $100=$ very certain. Higher scores indicate higher self-efficacy.

Reliability coefficients for the BPSES subscales range from 0.88 to 0.90 (Anderson, 1995). The BPSES has shown good construct and concurrent validity (Anderson et al., 1995; Levin et al., 1996) and was used by Williams, et al. (2005) in evaluating the effects of Iyengar yoga on cLBP (Williams, et al., 2005). Norms are not yet established for evaluating clinically important change when using the BPSES.

\section{Fear of Movement}

Pain related fear of movement was assessed with the Tampa Scale of Kinesphobia (TSK)(Appendix F). The TSK was developed to measure fear of work-related activities and fear of movement/re-injury (Miller, Kori, \& Todd, 1991; Swinkels-Meewisse, Swinkels, Verbeek, Vlaeyen, \& Oostendorp, 2003; Vlaeyen et al., 1995). The TSK is a 17-item, self-administered questionnaire which takes approximately five minutes to complete and requires an $8^{\text {th }}$ grade reading level. Each item is rated on a 4 point Likert scale ranging from $1=$ strongly disagree to $4=$ strongly agree. A total score is calculated after inverting the individual scores of items 4, 8, 12, and 16. Scores range from 17-68 with higher scores indicating greater pain-related fear. Scores $\geq 37$ indicate a high level of kinesiophobia. A 30\% pre-post change score reduction is considered clinically relevant (Vlaeyen, de Jong, Geilen, Heuts, \& van Breukelen, 2001).

Internal consistency of the TKS ranges from $\alpha=.77$ to .83 (Swinkels-Meewisse et al., 2003; Vlaeyen et al., 1995), and reliability coefficients range from .64-.80 in a back pain population (Swinkels-Meewisse et al., 2003). The TKS has demonstrated good concurrent validity in detecting persons whose disability from cLBP is related to fear rather than to pain (Vlaeyen et al., 1995). This instrument was also used by Williams and colleagues (2005). 


\section{Pain Medication Usage}

Pain medication usage was measured at baseline by having participants average their use of all prescription, non-prescription, herbal and dietary supplements to relieve pain during the two weeks prior to the start of yoga or physical therapy. Changes in usage from baseline were reassessed at 6 weeks by asking patients to average all analgesic pain medication used in the prior two weeks. Participants were also asked if they thought they were using more, the same or less pain medication at 6 and 12 weeks compared to their baseline use. This method was used to assess changes in medication use by other cLBP yoga researchers (Sherman, et al., 2005; Williams et al., 2005).

\section{Treatment Adherence}

Participants were asked to record the total number of minutes of home practice each week in a practice $\log$ collected weekly during the intervention period. Each participant's average number of minutes of home practice during the treatment period was determined by averaging the minutes of home practice from each of the weekly practice logs. The practice log is included in Appendix G. Class attendance for yoga participants and the total number of hours of physical therapy attended by PT participants within 6 weeks were compared to assess differences in the number of total treatment hours for the groups. Treatment adherence at 12 weeks was assessed by having participants average the number of minutes of weekly home practice since the 6 week assessment.

\section{Pain}

Each participant was asked to estimate their total duration of cLBP in months at baseline. Pain frequency was measured at baseline, 6 and 12 weeks by asking participants to average the number of days they experienced back pain during the previous 2 weeks.

Pain bothersomeness was measured at baseline, 6 and 12 weeks using an 11-point $(0-10)$ Likert scale where $0=$ not at all bothersome and $10=$ extremely bothersome (Appendix H). Participants were asked to rate their average pain bothersomeness over the preceding two weeks and their worst pain bothersomeness in the past month and in the past 6 months.

This method of assessing pain interference has shown construct validity (Patrick et al., 1995) as well as responsiveness in assessing pain and disability in cLBP populations (Stewart et al., 1994) and is one of the recommended methods for assessing clinical back pain treatment effects (Deyo et al., 1998; Von Korff et al., 2000). Similar pain bothersomeness scales were used by Sherman et al., (2005) and Uyterhoeven, Khalsa, \& Whittemore (2008) when evaluating the effects of yoga on cLBP. According to Farrar, Young, LaMoreaux, Werth, \& Poole (2001), a change score of 1.5 on an 11point bothersomeness of pain scale is clinically significant. 


\section{Treatment Satisfaction}

Treatment satisfaction was assessed at 6 and 12 weeks by asking participants to rate satisfaction with care using a 5-point Likert scale, where $0=$ not at all satisfied and $5=$ completely satisfied. Satisfaction with care is considered a core domain associated with improved back treatment outcomes (Deyo et al., 1998; Evans et al., 2004).

Questions regarding treatment satisfaction were included in the 6 week Short Term Yoga Program (Appendix I) and 6 week Short Term Physical Therapy (Appendix J) follow-up questionnaires. Satisfaction with care was also measured at 12 weeks in the 12 week Long-Term Yoga and Physical Therapy Follow-up Questionnaire (Appendix K).

\section{Conceptual Model with Study Variables and Variable Relationships}

A modification of the Disablement Model, as shown in Figure 3.2, includes the study variables, instruments and concept relationships.

\section{Description of Group Interventions}

\section{Yoga}

Yoga participants were recruited from a total of five separate 6-week Integral yoga classes beginning in January 2008 and concluding in September 2008. Each class met once a week for two hours. All participants were required to be medically screened by a healthcare provider prior to enrolling. Participants self-enrolled at a cost of $\$ 75.00$ for the six-week series. Class sizes varied throughout the intervention period, ranging from 6 to 12 participants with some participants electing not to volunteer for participation in the research study.

The Integral back yoga intervention was developed by a Senior Integral yoga therapist and instructor, the clinic's medical director, a physician and certified Integral yoga instructor, in collaboration with several physical therapists from the Athens Regional Medical Center Rehabilitation Department.

Integral yoga, considered a safe and gentle method of yoga, is based on the teachings of Sri Sachidananda (1990). The method emphasizes the therapeutic relationship between the teacher and the student, classical yoga principles and specific methods to promote tissue healing and enhance emotional well-being (M. Spears, personal communication, November 6, 2008). Each class session incorporates a series of modified postures (asanas), breath control techniques (pranayama), deep relaxation (yoga nidra) and mindfulness-based meditation components. 


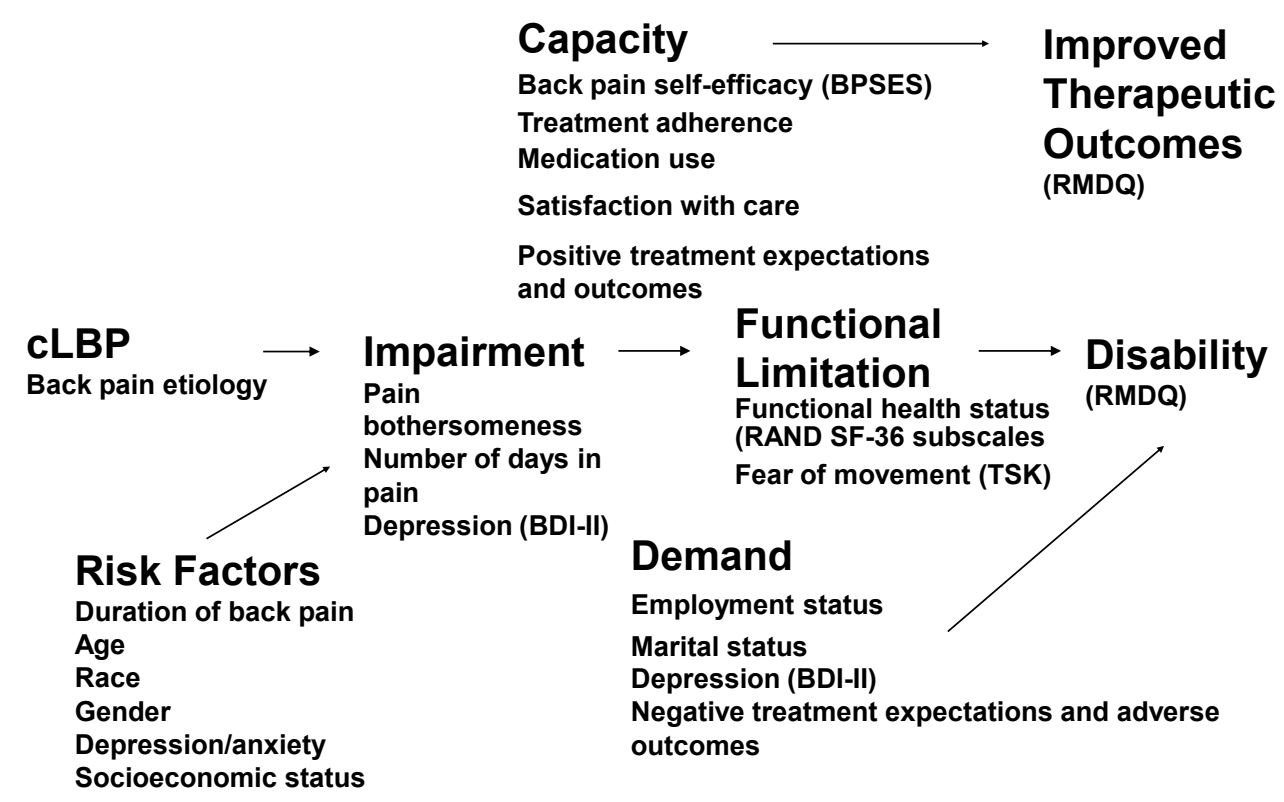

Figure 3.2 Modified Disablement Model with Study Variables, Instruments and Concept Relationships.

BPSES $=$ Back Pain Self-Efficacy Scale

$\mathrm{RMDQ}=$ Roland Morris Disability Questionnaire

$\mathrm{cLBP}=$ Chronic low back pain

BDI-II = Beck Depression Inventory-II

RAND SF-36 = Rand Short-Form 36-Item Health Survey

TSK $=$ Tampa Scale of Kinesiophobia

Permission to use Disablement Model from Elsevier; Verbrugge, L. M., \& Jette, A. M. (1994). The disablement process. Social Science and Medicine, 38(1), 1-14. Modified by permission (Alan Jette, personal communication, October 1, 2008). 
The modified postures developed for the back yoga classes were designed to strengthen muscles, stretch joints, improve balance and increase awareness of core spinal stabilizers and other muscles engaged during spinal movements. Performing postures mindfully by linking breath with movements is emphasized to focus attention on the sensations experienced during movements, to reduce anxiety and fear and reduce the risk of over-stretching and re-injury. The yoga poses developed for the back yoga series are included in Appendix L.

Breath control techniques are included in each class to improve concentration, increase oxygenation to tissues and enhance periods of deep relaxation (yoga nidra). Periods of yoga nidra are interspersed throughout the 2 hour class session to provide rest, promote tissue healing and to integrate the neuromuscular and proprioceptive repatterning which occurs during the execution of controlled breathing and postural movements (M. Spears, personal communication, November 6, 2008).

Yoga classes were taught by an experienced, Integral yoga instructor certified to teach therapeutic yoga to persons with cLBP. Each class began with a period of group sharing to discuss specific problems or concerns. This was followed by 10 minutes of deep breathing and relaxation performed seated in a chair or lying down on the floor. Props, including pillows, blankets and bolsters, were provided as needed for comfort. After this initial relaxation period yoga postures to warm up the spine were practiced for 30 minutes. These postures were performed on the floor in either supine or prone positions; then the class transitioned to standing poses or visa versa.

Each posture was introduced with individualized modifications to reduce anxiety and pain. The selection of postures chosen for each class was designed to be flexible and to vary according to individual participant needs, which were reviewed at the beginning of each class session. Props such as straps, blocks, blankets and bolsters were provided if needed for individuals to modify poses to reduce pain and prevent injury.

A second 10-minute period of deep relaxation followed the initial series of postures to provide a period of rest and to transition from standing poses to poses performed on the floor or visa versa. During this relaxation period, participants were instructed to pay attention to physical sensations that may have changed following the asana practice. This was emphasized to increase body awareness and reduce anxiety.

The second phase of postures was then performed using modifications as needed for 25 minutes before concluding with 10 to 15 minutes of chair or floor-seated meditation and pranayama breathing exercises. The pranayama exercises introduced during this period included three part breathing and alternate nostril and Kapalabhati breathing techniques. These breathing techniques activate and help tone core abdominal muscles involved in spinal support and stabilization (Michels et al., 2006)). Participants are instructed to become aware of how these core muscles feel during breathing exercises, relaxation and when moving the spine during asana practice. A 15 to 20 minute period of deep relaxation performed in corpse pose or savasana concluded each class session. 
At the conclusion of each class the yoga teacher provided handouts of suggested home practice yoga exercises. Participants were also reminded to complete their weekly home practice and pain logs that were collected each week by the investigator prior to the start of class. A \$10.00 incentive was provided to participants after they completed the 6 week assessment questionnaires following the last yoga class.

\section{Physical Therapy}

Physical therapy sessions were individualized, averaging 45 to 60 minutes in length. Sessions were conducted individually by a licensed physical therapist or physical therapy assistant one to two days a week for 6 weeks. The total duration of treatment for each participant varied according to their response to treatment and duration of treatment based on insurance reimbursement criteria. Session costs varied according to session length and if additional modalities such as electrical muscle stimulation methods, ultrasound or thermal treatments were used. The average cost of a one hour PT session was based on a 15 minute unit cost that ranged from $\$ 35.00$ to $\$ 73.00 /$ unit. Charges varied with use of adjunctive treatment modalities (V. Dillow, personal communication, November 10, 2008; C. Doerr, personal communication, December 18, 2008).

Because PT participants were recruited from two different sites, there were seven different licensed physical therapists conducting the individualized PT sessions. However, each participant was treated by only one primary physical therapist throughout their treatment course. Each physical therapist assigned weekly home practice exercises, collected weekly pain and practice logs and administered the 6 week assessment questionnaires. Participants were awarded $\$ 10.00$ after completing the 6 week questionnaires.

Both PT clinics offer an exercise-based program for cLBP rehabilitation. At the Physician's Back and Neck Clinic, PT sessions are conducted in a gym setting using resistive equipment developed by Med X (www.medxonline.co.uk). This equipment is designed to isolate, stabilize and strengthen specific muscle groups involved in the support of the lumbar spine to increase muscle size, proprioceptive tone and joint flexibility.

PT participants were instructed to perform the exercises using the SuperSlow technique (www.superslow.com), which consists of performing 20 second repetition cycles for a total of 120 seconds per MedX machine. This method is thought to enhance neuromuscular retraining of abdominal and lumbar muscles, which encourages awareness of the muscle sensations experienced during recruitment and execution of spinal movements to improve back function (C. Doerr, personal communication, June 30, 2007).

In addition to the use of resistive equipment, participants at the Physician's Back and Neck Clinic are instructed in total motion release (TMR) neuromuscular retraining 
(www.totalmotionrelease.com) and in transverse abdominus core strengthening exercises. These exercises are emphasized for weekly home practice. Pilates mat exercises (Isacowitz, 2006) are also introduced for home practice as participants gain strength. Manual therapies, including massage and spinal adjustment, electrical muscle stimulation methods, ultrasound and thermal treatments are added in cases of symptom exacerbation.

PT sessions conducted at the Athens Regional Medical Center Rehabilitation Department incorporate multiple modalities to reduce pain and improve back function including back exercises, back education, McKenzie method techniques and passive pain reducing modalities including thermal treatments and ultrasound. Cybex equipment is also used to build strength.

The back education component is designed to increase participant awareness of abnormal movement patterns and to improve posture and use of proper body mechanics to reduce pain and risk of re-injury. Individualized back exercise treatment plans for home practice were developed for each participant. These plans included daily exercises to increase back strength and flexibility. Home exercise practice was emphasized weekly, and exercises were added in a graded fashion as pain and strength improved ( $\mathrm{T}$. Story, RT, personal communication, November 12, 2008).

\section{Data Collection Procedures}

Informed consent and agreement to participate throughout the duration of the study was obtained during enrollment for yoga participants and for physical therapy participants prior to their first scheduled physical therapy session. All participants completed baseline questionnaires prior to the start of PT or the first yoga class to maintain a pre-post research design. Five weekly practice and pain logs were completed, starting at the first week of treatment and concluding after the $6^{\text {th }}$ week for both groups. Twelve weeks after the start of either PT or the first yoga class participants were contacted by phone and to complete the long-term follow-up questionnaire. Table 3.1 shows the outcome measures and assessment periods used in this study.

\section{Data Management Procedures}

All questionnaires were kept in secure locations within each clinic and checked for missing data and accuracy before being entered into a fire-wall protected computer for later analysis using the Statistical Package for the Social Sciences version 16 (SPSS ${ }^{\circledR}$ Inc., Chicago, IL). In cases of missing data study participants were contacted by phone to complete the missing items. In the rare cases when participants were unable to be contacted missing item values were imputed by calculating the item mean of the total sample and then using that value for the group data analysis. 
Table 3.1 Assessment Procedures.

\begin{tabular}{|c|c|c|c|c|}
\hline Variables & Baseline & Intervention & $\begin{array}{c}6 \text { Week } \\
\text { Follow up }\end{array}$ & $\begin{array}{c}12 \text { Week } \\
\text { Follow up }\end{array}$ \\
\hline \multicolumn{5}{|l|}{ Baseline Demographic } \\
\hline Questionnaire & $\mathrm{X}$ & & & \\
\hline RMDQ & $X$ & & $\mathrm{X}$ & \\
\hline BDI-II & $X$ & & $X$ & \\
\hline RAND SF-36 1.0 & $\mathrm{X}$ & & $\mathrm{X}$ & \\
\hline BPSES & $\mathrm{X}$ & & $\mathrm{X}$ & \\
\hline TSK & $\mathrm{X}$ & & $\mathrm{X}$ & \\
\hline \multicolumn{5}{|l|}{ Short Term Follow-up } \\
\hline Questionnaire & & & $X$ & \\
\hline \multicolumn{5}{|l|}{ Long Term Follow-up } \\
\hline Questionnaire & & & & $X$ \\
\hline \multicolumn{5}{|l|}{ Weekly Practice and Pain } \\
\hline $\log$ & & $\mathrm{X}$ & $\mathrm{X}$ & $X$ \\
\hline \multicolumn{5}{|l|}{ Pain Characteristics } \\
\hline Questionnaire & $\mathrm{X}$ & $\mathrm{X}$ & $\mathrm{X}$ & $\mathrm{X}$ \\
\hline $\begin{array}{l}\text { RMDQ = Roland Morris D } \\
\text { BDI-II = Beck Depression } \\
\text { RAND SF-36 } 1.0=\text { Rand S } \\
\text { BPSES = Back Pain Self-E } \\
\text { TSK = Tampa Scale of Kin }\end{array}$ & $\begin{array}{l}\text { ability Ques } \\
\text { ventory ver } \\
\text { prt-Form } 36 \\
\text { icacy Scale } \\
\text { iophobia }\end{array}$ & $\begin{array}{l}\text { onnaire } \\
\text { on } 2 \\
\text { tem Health Su }\end{array}$ & 1.0 & \\
\hline
\end{tabular}




\section{Data Analysis}

Statistical analysis was performed using SPSS, the Statistical Package for the Social Sciences, version 16 (SPSS ${ }^{\circledR}$ Inc., Chicago, IL). A significance level of $p \leq 0.05$ was considered to indicate a significant difference for all tests. Descriptive statistics were run for all continuous data to obtain means, standard deviations and ranges. Frequencies and percentages were run for categorical and nominal variables. Normality assessments indicated that many of the variables were positively skewed. Because of the small sample sizes and variable distributions that were not normally distributed, non-parametric statistics were used for within and between group comparisons of continuous variables. Chi-square methods were used for group comparisons of nominal and categorical variables. For clarity, each aim and associated research questions are presented, followed by the specific methods used in the data analysis.

\section{Specific Aim 1}

To compare pre-existing demographic and clinical characteristics of yoga and physical therapy groups. Following are the associated research questions and methods:

1.1 What are the differences between the groups in the following demographic characteristics: (a) gender, (b) age, (c) income, (d) educational level, (e) marital status and (f) employment status?

Mann Whitney U tests were used to compare mean rank differences between the groups in age and educational level. Chi-square analysis was used to compare group differences in marital status, income, employment status and gender.

1.2 What are the baseline differences between the groups on the psychological variables (a) depression (BDI-II), (b) pain self-efficacy (BPSES) and (c) fear of movement (TSK)?

Mann Whitney U tests were used to compare mean rank baseline differences between groups on the BDI-II, BPSES and TSK.

1.3 What are the baseline differences between the groups in functional disability (RMDQ)?

A Mann Whitney U test was used to compare the baseline group differences on the RMDQ.

1.4 What are the baseline differences between the groups in health status (RAND SF-36 1.0 general health, bodily pain, social function and physical function subscales)? 
Mann Whitney U tests were used to compare the mean rank baseline differences between the groups on each of the RAND SF-36 1.0 subscale scores.

1.5 What are the baseline differences between the groups in pain-related variables considering (a) total duration of cLBP in weeks, (b) pain frequency (number of days in pain per week), (c) pain bothersomeness (worst and average), (d) average pain medication usage (doses per week) and (e) reduced activity related to pain (days per week)?

Mann Whitney U tests were used to compare mean rank baseline differences between the groups on pain-related variables.

1.6 What are the baseline differences between the groups in treatment-related variables considering treatment expectations and reason for treatment selection?

Chi-square analysis was used to compare the baseline differences in treatment expectations and reason for treatment selection.

\section{Specific Aim 2}

To compare treatment outcomes of yoga and physical therapy groups.

2.1 What are the differences between the groups on psychological outcomes at 6 weeks (BDI-II, BPSES and TSK)??

The within group baseline to 6 week, mean rank change score differences on the BDI-II, BPSES and TSK were compared using Wilcoxin Signed Ranks test for each outcome measure. The mean rank change score differences were then compared between the groups using Mann Whitney U tests to determine the group differences.

2.2 What are the differences between groups in functional disability at 6 weeks (RMDQ)?

Within group mean rank differences from baseline to 6 weeks in disability were compared using the Wilcoxin Signed Ranks test. Mean rank change score differences between groups were compared using a Mann Whitney U test.

2.3 What are the differences between the groups in health status at 6 weeks (RAND SF-36 1.0 general health, bodily pain, social function and physical function subscale scores)? 
Within group mean rank differences from baseline to 6 weeks in health status subscale scores were compared using Wilcoxin Signed Ranks tests. Mean rank change score differences between groups were compared using Mann Whitney U tests.

2.4 What are the differences between the groups in pain-related variables at 6 and 12 weeks considering (a) average number of days in pain in past 2 weeks, (b) average number of days with reduced activity due to pain in past month, (c) average pain bothersomeness in past 2 weeks, (d) worst pain bothersomeness in past month and in past 6 month and (e) average pain medication usage?

Within group differences in pain-related variables were compared using Wilcoxin Signed Ranks tests. Mean rank change score differences from baseline to 6 weeks and baseline to 12 weeks between the groups were then compared using Mann Whitney U tests. Chi-square analysis was performed to determine group differences in pain medication usage from baseline to 6 and baseline to 12 weeks.

2.5 What are the differences between the groups in treatment-related variables at 6 and 12 weeks considering (a) minutes per week of home practice, (b) total duration of treatment in hours during the intervention period, (c) treatment satisfaction and (d) treatment benefits.

Within group differences in minutes of home practice at 6 and 12 weeks was compared using Wilcoxin Signed Ranks tests. Mean rank between group differences in minutes of home practice at 6 and 12 weeks and treatment duration were compared using Mann Whitney $\mathrm{U}$ tests. Chi-square analysis was used to compare the groups at 6 and 12 weeks in treatment satisfaction and treatment benefits.

\section{Consideration of Human Subjects}

Approval to conduct this study was obtained from the Institutional Review Committees of the University of Tennessee Health Science Center (IRB) and Athens Regional Medical Center prior to data collection. The IRB approval forms are included in Appendix M. Procedures to ensure anonymity and confidentiality for study participants were strictly maintained. Questionnaires were labeled with de-identifying codes for each participant. Data collection forms linking the participant's name and phone number with identification codes were kept in secure locations within each clinic. All data analysis was based on aggregate data, and in no cases were participant names reported.

The study posed minimal risks to participants. Participation in the study was voluntary and offered to all interested individuals who met inclusion criteria after selfenrolling into yoga or PT for treatment based on healthcare provider or self-referral. 


\section{CHAPTER 4. RESULTS}

The purpose of this study was to compare the characteristics of individuals who self-select yoga with those who self-select physical therapy for treatment of CLBP and to examine treatment outcomes. The findings from this study are organized and discussed by study aim and associated research questions.

\section{General Description of the Sample}

A convenience sample of 53 adults ages 18 and older, who reported a history of cLBP $>12$ weeks in duration participated in this study. A total of 27 adults enrolling in Integral yoga classes volunteered to participate and completed baseline questionnaires. Twenty-one of these participants completed the 6 and 12 week assessment questionnaires. Twenty-six participants recruited from two physical therapy clinics volunteered to participate and completed baseline questionnaires. Nine participants were recruited at the Athens Regional Medical Center Rehabilitation PT department, and 17 participants were recruited from the Physician's Back and Neck Clinic. Twenty-one PT participants completed the 6 week assessment questionnaires and 16 completed the 12 week assessment questionnaires. Five PT participants were unable to be located to complete the 12 week assessment questionnaires. Table 4.1 summarizes the reasons for attrition at 6 weeks for both groups.

\section{Aim 1-To Describe Group Differences at Baseline}

Clinical and demographic characteristics of PT and yoga were compared using Mann Whitney $U$ tests for continuous variables and Chi-square analysis for nominal and categorical variables. There were significant differences between the groups in race, working status and income level. PT participants were more likely to be nonwhite, earning $\leq \$ 30,000$ annually and not working or receiving disability. Yoga participants were more likely to be employed and earning $\geq \$ 70,000$. Group comparisons are shown in Table 4.2.

Chi-square analyses of treatment expectations and reason for treatment choice showed significant differences between the groups. Yoga participants were more likely to have chosen yoga after recommendations from friends (59\%) or after having read about it (52\%), while PT participants were more likely to have chosen PT because of healthcare provider recommendation (85\%) and insurance coverage (50\%). Each group selected reduced pain as the most important treatment benefit; however, yoga participants were more likely to choose increased flexibility as an important treatment benefit and PT participants were more likely to select reduced pain medication use. Group comparisons of treatment-related characteristics are shown in Tables 4.3 and 4.4. 
Table 4.1 Reasons for Attrition.

\begin{tabular}{|c|c|}
\hline Yoga $(n=6)$ & PT $(n=5)$ \\
\hline 3 - class time inconvenient & $\begin{array}{l}2 \text { - failed to return after completing baseline } \\
\text { questionnaires }\end{array}$ \\
\hline $\begin{array}{l}1 \text { - entered rehabilitation to wean } \\
\text { off narcotic analgesics }\end{array}$ & 1 - physician ordered different treatment \\
\hline 1 - class was different than expected & 1 - got better by second treatment \\
\hline $1-$ no show after first class & $1-$ quit due to insurance reimbursement problem \\
\hline
\end{tabular}

Table 4.2 Baseline Characteristics of Groups.

\begin{tabular}{|c|c|c|c|}
\hline Characteristics & $\begin{array}{c}\text { Yoga } \\
(n=27)\end{array}$ & $\begin{array}{c}\text { PT } \\
(n=26)\end{array}$ & $\begin{array}{c}X^{2} \text { or Mann } \\
\text { Whitney U } \\
p \text { Value }\end{array}$ \\
\hline & $\mathrm{M} \pm \mathrm{SD}$ & $\mathrm{M} \pm \mathrm{SD}$ & \\
\hline Mean age in years & $49.3 \pm 14.04$ & $52.1 \pm 17.16$ & $p=.101$ \\
\hline Mean years of education & $17.3 \pm 2.45$ & $14.7 \pm 2.45$ & $p=.188$ \\
\hline $\begin{array}{l}\text { Mean duration of cLBP in } \\
\text { months }\end{array}$ & $102.3 \pm 86.95$ & $115.2 \pm 137.26$ & $p=.978$ \\
\hline Gender & $\mathrm{N}(\%)$ & $\mathrm{N}(\%)$ & \\
\hline Male & $8(30 \%)$ & $10(39 \%)$ & $p=.497$ \\
\hline Female & $19(70 \%)$ & $16(62 \%)$ & \\
\hline \multicolumn{4}{|l|}{ Race } \\
\hline White & $26(96 \%)$ & $15(75 \%)$ & $p=.031$ \\
\hline Nonwhite & $1(3.7 \%)$ & $5(25 \%)$ & \\
\hline \multicolumn{4}{|l|}{ Relationship status } \\
\hline Married & $19(70 \%)$ & $13(65 \%)$ & $p=.696$ \\
\hline Unmarried & $8(30 \%)$ & $7(35 \%)$ & \\
\hline \multicolumn{4}{|l|}{ Annual income level } \\
\hline$\$ 30,000$ or less & $5(19 \%)$ & $9(38 \%)$ & \\
\hline$\$ 31,000-\$ 69,000$ & $11(41 \%)$ & $10(42 \%)$ & $p=.194$ \\
\hline$\$ 70,000$ or greater & $11(41 \%)$ & $5(21 \%)$ & \\
\hline \multicolumn{4}{|l|}{ Employment status } \\
\hline Not working & $6(22.0 \%)$ & $9(36.0 \%)$ & \\
\hline Working full or part-time & $20(74 \%)$ & $12(48 \%)$ & $p=.115$ \\
\hline Receiving disability & $1(3.7 \%)$ & $4(16 \%)$ & \\
\hline \multicolumn{4}{|l|}{ Back pain etiology } \\
\hline Disc disease & $11(41 \%)$ & $11(42 \%)$ & $p=.908$ \\
\hline Muscle strain & $8(30 \%)$ & $2(8 \%)$ & $p=.041$ \\
\hline Sciatica & $6(22 \%)$ & $8(31 \%)$ & $p=.480$ \\
\hline Spinal stenosis & 0 & $6(23 \%)$ & $p=.008$ \\
\hline
\end{tabular}


Table 4.3 Pre-Treatment Reasons for Choosing PT or Yoga.

\begin{tabular}{|c|c|c|c|}
\hline Reasons & $\begin{array}{c}\text { Yoga } \\
(\mathrm{n}=27)\end{array}$ & $\begin{array}{c}\text { PT } \\
(n=26)\end{array}$ & $\begin{array}{c}X^{2} \\
p \text { Value }\end{array}$ \\
\hline & $\mathrm{N}(\%)$ & $\mathrm{N}(\%)$ & \\
\hline Covered by insurance & $1(4 \%)$ & $13(50 \%)$ & $p=.000$ \\
\hline Cost & $2(7 \%)$ & 0 & $p=.096$ \\
\hline $\begin{array}{l}\text { Recommended by } \\
\text { healthcare provider }\end{array}$ & $1(4 \%)$ & $22(85 \%)$ & $p=.000$ \\
\hline $\begin{array}{l}\text { Recommended by } \\
\text { others }\end{array}$ & $16(59 \%)$ & $6(23 \%)$ & $p=.008$ \\
\hline Read about it & $14(52 \%)$ & 0 & $p=.000$ \\
\hline
\end{tabular}

$\mathrm{PT}=$ physical therapy

Table 4.4 Pre-Treatment Expectations.

\begin{tabular}{|c|c|c|c|}
\hline Expectations & $\begin{array}{c}\text { Yoga } \\
(n=27)\end{array}$ & $\begin{array}{c}\text { PT } \\
(n=26)\end{array}$ & $\begin{array}{c}X^{2} \\
p \text { Value }\end{array}$ \\
\hline & $\mathrm{N}(\%)$ & $\mathrm{N}(\%)$ & \\
\hline Reduced pain & $24(89 \%)$ & $26(100 \%)$ & $p=.248$ \\
\hline Increased strength & $20(74 \%)$ & $16(61.5 \%)$ & $p=.328$ \\
\hline Increased flexibility & $25(93 \%)$ & $17(65 \%)$ & $p=.015$ \\
\hline Reduced stress & $13(48 \%)$ & $11(43 \%)$ & $p=.669$ \\
\hline $\begin{array}{l}\text { To reduce pain medication } \\
\text { usage }\end{array}$ & $9(33 \%)$ & $17(65 \%)$ & $p=.020$ \\
\hline
\end{tabular}


Mann Whitney U tests were used to test mean group differences in psychological variables. The groups differed significantly in pain self-efficacy and fear of movement. Yoga participants had significantly greater pain self-efficacy and less fear of movement, and both groups scored in the high fear of movement range (scores $>37$ ) according to Vlaeyen (1995). There was no significant difference between the groups on the BDI-II. Both groups scored in the normal to low range of depressive symptomotology. Refer to Table 4.5.

Mann Whitney U tests were used to compare the groups in health status and disability measures. The results showed that yoga participants were significantly less disabled and had significantly higher health status scores compared to PT participants. This indicates that yoga participants were less functionally impaired at baseline compared to the PT participants suggesting they were experiencing less acute symptoms. Group comparisons of health status and disability are shown in Table 4.6.

Baseline differences between the groups in pain characteristics were analyzed using Mann Whitney U tests, and significant group differences were found. PT participants reported more days in pain, more days with reduced activity related to pain and significantly greater pain medication usage compared to yoga participants. PT participants also had significantly greater average and worst pain bothersomeness in the month preceding treatment compared to yoga participants; although there was no significant difference between the groups in worst pain bothersomeness 6 months prior to treatment. These findings are consistent with the differences in disability and function found between the groups on the health status and disability measures. Group differences in pain characteristics are shown in Table 4.7.

\section{Aim 2-To Compare Treatment Outcomes}

Differences between the groups at 6 weeks in psychological characteristics were analyzed by calculating within group baseline to 6 week change scores and comparing the mean ranks using Mann Whitney U tests. Within group changes were analyzed using Wilcoxin Signed Ranks tests. The within group analysis found that both groups had significantly reduced depression scores and reduced fear of movement scores at 6 weeks following treatment. However, fear of movement was only significantly reduced for the PT group, and neither group reached the $30 \%$ change score criterion indicative of clinically significant reduced fear of movement (Vlaeyen et al., 2001). The groups also had improved pain self-efficacy scores at 6 weeks; however, only the yoga participants improved significantly. Baseline to 6 weeks change score differences between the groups were not found to differ significantly on any of the psychological measures. The within and between group changes in psychological variables are shown in Tables 4.8 and 4.9, respectively.

Within group changes in health status and disability at 6 weeks were tested using Wilcoxin Signed Ranks tests and between group differences in change scores were compared, using Mann Whitney $U$ tests. The within group analysis found that both 
Table 4.5 Pre-Treatment Group Comparisons of Psychological Variables.

\begin{tabular}{lccc}
\hline $\begin{array}{c}\text { Psychological } \\
\text { Questionnaires }\end{array}$ & $\begin{array}{c}\text { Yoga } \\
(\mathrm{n}=27)\end{array}$ & $\begin{array}{c}\mathrm{PT} \\
(\mathrm{n}=26)\end{array}$ & $\begin{array}{c}\text { Mann Whitney U } \\
p \text { Value }\end{array}$ \\
\hline & $\underline{\mathrm{M} \pm \mathrm{SD}}$ & $\underline{\mathrm{M} \pm \mathrm{SD}}$ & \\
Beck Depression Inventory-v2 & $8.6 \pm 7.05$ & $10.7 \pm 9.38$ & $p=.232$ \\
Tampa Scale for Kinesiophobia & $39.3 \pm 9.49$ & $44.6 \pm 5.52$ & $p=.033$ \\
Self-Efficacy Pain Subscale & $76.1 \pm 18.23$ & $59.5 \pm 59.46$ & $p=.012$ \\
\hline
\end{tabular}

$\mathrm{PT}=$ physical therapy

Table 4.6 Pre-Treatment Group Comparisons of Health Status and Disability.

\begin{tabular}{|c|c|c|c|}
\hline $\begin{array}{l}\text { Psychological } \\
\text { Questionnaires }\end{array}$ & $\begin{array}{c}\text { Yoga } \\
(n=27)\end{array}$ & $\begin{array}{c}\text { PT } \\
(\mathrm{n}=26)\end{array}$ & $\begin{array}{c}\text { Mann Whitney } \mathrm{U} \\
p \text { Value }\end{array}$ \\
\hline & $\mathrm{M} \pm \mathrm{SD}$ & $\mathrm{M} \pm \mathrm{SD}$ & \\
\hline RMDQ & $\overline{5.4 \pm 4.76}$ & $9 . \overline{23 \pm 5.90}$ & $p=.013$ \\
\hline RAND SF-36 1.0 Bodily & & & \\
\hline $\begin{array}{l}\text { Pain } \\
\text { RAND SF-36 1.0 Physical }\end{array}$ & $55.4 \pm 19.17$ & $36.1 \pm 19.47$ & $p=.001$ \\
\hline $\begin{array}{l}\text { Function } \\
\text { RAND SF-36 1.0 Social }\end{array}$ & $65.8 \pm 23.52$ & $48.26 \pm 27.85$ & $p=.023$ \\
\hline $\begin{array}{l}\text { Function } \\
\text { RAND SF-36 } 1.0 \text { General }\end{array}$ & $77.3 \pm 25.48$ & $57.69 \pm 25.01$ & $p=.004$ \\
\hline Health & $67.0 \pm 18.77$ & $59.20 \pm 20.23$ & $p=.169$ \\
\hline
\end{tabular}

$\mathrm{PT}=$ physical therapy

RMDQ = Roland Morris Disability Questionnaire

RAND SF-36 1.0= Rand Short-Form 36-Item Health Survey 1.0 
Table 4.7 Pre-Treatment Differences in Pain-Related Variables.

\begin{tabular}{|c|c|c|c|}
\hline Pain-Related Variables & $\begin{array}{c}\text { Yoga } \\
(n=27)\end{array}$ & $\begin{array}{c}\text { PT } \\
(n=26)\end{array}$ & $\begin{array}{c}\text { Mann Whitney } \mathrm{U} \\
p \text { Value }\end{array}$ \\
\hline $\begin{array}{l}\text { Worst pain bothersomeness } \\
\text { (past } 6 \text { months) } \\
\text { (past } 4 \text { weeks) }\end{array}$ & $\begin{array}{l}\underline{M} \pm \mathrm{SD} \\
7.7 \pm 2.14 \\
6.5 \pm 2.60\end{array}$ & $\begin{array}{l}\underline{\mathrm{M} \pm \mathrm{SD}} \\
8.5 \pm 1.60 \\
8.3 \pm 1.48\end{array}$ & $\begin{array}{l}p=.113 \\
p=.008\end{array}$ \\
\hline $\begin{array}{l}\text { Average days in past month cut } \\
\text { down on usual activities because } \\
\text { of back pain }\end{array}$ & $8.7 \pm 10.93$ & $14.0 \pm 11.36$ & $p=.039$ \\
\hline (past 2 weeks) & $9.7 \pm 5.21$ & $13.0 \pm 2.28$ & $p=.010$ \\
\hline $\begin{array}{l}\text { Average pain bothersomeness } \\
\text { (past } 2 \text { weeks) } \\
\text { Average doses of pain medication } \\
\text { (past } 2 \text { weeks) }\end{array}$ & $\begin{array}{l}4.5 \pm 2.37 \\
9.3 \pm 12.89\end{array}$ & $\begin{array}{c}7.5 \pm 1.83 \\
22.2 \pm 19.80\end{array}$ & $\begin{array}{l}p=.000 \\
p=.018\end{array}$ \\
\hline
\end{tabular}

$\mathrm{PT}=$ physical therapy 
Table 4.8 Within Group Changes on Psychological Measures at 6 Weeks.

\begin{tabular}{lcccccc}
\hline Measure & $\begin{array}{c}\text { Yoga } \\
\text { Baseline } \\
(\mathrm{n}=21)\end{array}$ & $\begin{array}{c}\text { Yoga } \\
6 \text { Weeks } \\
(\mathrm{n}=21)\end{array}$ & $\begin{array}{c}* p \\
\text { Value }\end{array}$ & $\begin{array}{c}\text { PT } \\
\text { Baseline } \\
(\mathrm{n}=20)\end{array}$ & $\begin{array}{c}\text { PT } \\
6 \text { Weeks } \\
(\mathrm{n}=20)\end{array}$ & $\begin{array}{c}* p \\
\text { Value }\end{array}$ \\
\hline BDI-II & $\underline{\mathrm{M} \pm \mathrm{SD}}$ & $\underline{\mathrm{M} \pm \mathrm{SD}}$ & & $\underline{\mathrm{M} \pm \mathrm{SD}}$ & $\underline{\mathrm{M} \pm \mathrm{SD}}$ & \\
BPSES & $76.7 \pm 18.76$ & $81.4 \pm 5.65$ & .002 & $11.6 \pm 9.59$ & $7.5 \pm 9.78$ & .004 \\
TSK & $38.4 \pm 9.01$ & $36.4 \pm 10.32$ & .029 & $57.5 \pm 23.62$ & $66.3 \pm 23.64$ & .126 \\
\hline
\end{tabular}

*Means compared with Wilcoxin Signed Ranks Test

$\mathrm{PT}=$ physical therapy

BDI-II = Beck Depression Inventory-v2

BPSES = Back Pain Self-Efficacy Scale

TSK $=$ Tampa Scale of Kinesiophobia

Table 4.9 Between Group Psychological Change Score Differences at 6 Weeks.

\begin{tabular}{lccc}
\hline Measure & Yoga Change Score & PT Change Score & $\begin{array}{c}\text { Mann Whitney U } \\
p \text { Value }\end{array}$ \\
\hline BDI-II & $\frac{\mathrm{M} \pm \mathrm{SD}}{\overline{2} \pm 5.49}$ & $\underline{\mathrm{M} \pm \mathrm{SD}}$ & \\
BPSES & $4.8 \pm 9.76$ & $8.1 \pm 5.3$ & .927 \\
TSK & $2.0 \pm 5.75$ & $8.8 \pm 23.03$ & .870 \\
\hline
\end{tabular}

PT = physical therapy

BDI-II = Beck Depression Inventory-v2

BPSES = Back Pain Self-Efficacy Scale

TSK $=$ Tampa Scale of Kinesiophobia 
groups had improvements in health status with reduced disability scores at 6 weeks. Within group mean change scores also met the criterion for clinically significant improvement in disability at 6 weeks. Yoga participants who scored in the low range of disability RMDQ score $\leq 5$ at baseline had a mean change score of 1.57 on the RMDQ at 6 weeks and PT participants had a change score of 2.14. In less disabled populations (baseline scores less than 8) a change score of 1 to 2 points can be clinically relevant (Stratford et al., 1998). Change scores greater than 2 are considered significant in more disabled populations (baseline scores $\geq 9$ (Riddle, Stratford, \& Binkley, 1998).

Yoga participants also had significantly improved scores on the bodily pain, general health and physical function subscales of the RAND SF-36 1.0 and clinically significant change on all of the subscales with change scores $\geq 5$. Previous investigators have determined that change scores of 5 to 7 points on Rand-SF 36 subscales are clinically significant following back treatment interventions in cLBP populations (Deyo et al., 1998; Taylor et al., 1999). Yoga participants improved on the social function subscale but the change was not statistically significant.

PT participants obtained clinically and statistically significant improvements on the RAND SF-36 1.0 bodily pain, social function and physical function subscales. The PT group improved on the general health subscale but the change score difference at 6 weeks was not clinically or statistically significant. Within group baseline to 6 week differences are shown in Tables 4.10 and 4.11 for the yoga and PT group respectively.

There were no significant differences found between the groups when comparing mean change scores on the general health, physical function and social function subscales. There was a significant between group difference on the bodily pain subscale. The PT participants obtained a change score of 20 while the yoga participants obtained a change score of 9 which was clinically significant for both groups. Between group change score comparisons in disability and health status are shown in Table 4.12.

Outcome differences in pain characteristics across time were compared using Wilcoxin Signed Ranks tests for within group changes and Mann Whitney U tests for between group comparisons. Both groups had improvements in the average number of days in the past two weeks in pain from baseline to 6 weeks and from 6 weeks to 12 weeks. Yoga participants decreased from $9.70 \pm 5.21$ days at baseline to $6.90 \pm 5.18$ days at 6 weeks $(p \leq 0.05)$ and to $6.04 \pm 5.86$ days at 12 weeks $(p=0.134)$. PT participants decreased from $12.80 \pm 2.50$ days at baseline to $7.52 \pm 4.93$ days at 6 weeks $(p \leq 0.01)$ and to $6.73 \pm 5.67$ days at 12 weeks $(p \leq 0.01)$. Between group differences in the average number of days in pain in the past two weeks at baseline $(p \leq 0.01)$ were not statistically significant at 6 weeks $(p=0.809)$ or at 12 weeks $(p=0.772)$. Figure 4.1 shows the group changes in average number of days in pain across time.

The groups also had significant declines in the average number of days in the past month with reduced activity secondary to pain. The average number of days with reduced activity secondary to pain decreased for yoga participants from $8.74 \pm 5.21$ days at baseline, to $1.85 \pm 3.10$ days at 6 weeks $(p \leq 0.05)$ and to $0.47 \pm 0.98$ days at 12 weeks 
Table 4.10 Yoga within Group Disability and Health Status Differences at 6 Weeks.

\begin{tabular}{lcccc}
\hline \multicolumn{1}{c}{ Measure } & $\begin{array}{c}\text { Yoga } \\
\text { Baseline } \\
(\mathrm{n}=21)\end{array}$ & $\begin{array}{c}\text { Yoga 6 } \\
\text { Weeks } \\
(\mathrm{n}=21)\end{array}$ & $\begin{array}{c}\text { Change } \\
\text { Scores }\end{array}$ & $\begin{array}{c}\text { Wilcoxin } \\
\text { Signed } \\
\text { Ranks } \\
p \text { Value }\end{array}$ \\
\hline $\begin{array}{l}\mathrm{M} \pm \mathrm{SD} \\
\text { RMDQ }\end{array}$ & $4.33 \pm 3.61$ & $2.76 \pm 3.08$ & 1.57 & $p=.009$ \\
$\begin{array}{l}\text { RAND SF-36 1.0 } \\
\text { Bodily Pain }\end{array}$ & $57.3 \pm 19.07$ & $66.9 \pm 15.73$ & 9.64 & $p=.021$ \\
$\begin{array}{l}\text { RAND SF-36 1.0 } \\
\text { Physical Function }\end{array}$ & $68.8 \pm 22.52$ & $78.5 \pm 14.15$ & 9.75 & $p=.009$ \\
$\begin{array}{l}\text { RAND SF-36 1.0 } \\
\text { Social Function }\end{array}$ & $81.5 \pm 19.61$ & $89.9 \pm 14.58$ & 8.33 & $p=.068$ \\
$\begin{array}{l}\text { RAND SF-36 1.0 } \\
\text { General Health }\end{array}$ & $69.0 \pm 15.93$ & $74.3 \pm 16.75$ & 5.30 & $p=.041$ \\
\hline $\begin{array}{l}\text { RMDQ = Roland Morris Disability Questionnaire } \\
\text { RAND SF-36 1.0 }=\text { Rand Short-Form 36-Item Health Survey } 1.0\end{array}$ &
\end{tabular}

Table 4.11 PT within Group Disability and Health Status Differences at 6 Weeks.

\begin{tabular}{|c|c|c|c|c|}
\hline Measure & $\begin{array}{l}\text { PT Baseline } \\
\quad(\mathrm{n}=21)\end{array}$ & $\begin{array}{l}\text { PT } 6 \text { Weeks } \\
\quad(\mathrm{n}=21)\end{array}$ & $\begin{array}{l}\text { Change } \\
\text { Score }\end{array}$ & $\begin{array}{c}\text { Wilcoxin } \\
\text { Signed } \\
\text { Ranks } \\
p \text { Value }\end{array}$ \\
\hline & $\mathrm{M} \pm \mathrm{SD}$ & $\mathrm{M} \pm \mathrm{SD}$ & & \\
\hline $\begin{array}{l}\text { RMDQ } \\
\text { RAND SF-36 } 1.0\end{array}$ & $9 . \overline{1 \pm 5.29}$ & $7.0 \pm 5.67$ & 2.14 & $p=.026$ \\
\hline $\begin{array}{l}\text { Bodily Pain } \\
\text { RAND SF-36 } 1.0\end{array}$ & $37.1 \pm 20.90$ & $57.9 \pm 19.66$ & 20.83 & $p=.001$ \\
\hline $\begin{array}{l}\text { Physical Function } \\
\text { RAND SF-36 } 1.0\end{array}$ & $45.2 \pm 27.77$ & $55.9 \pm 27.23$ & 10.70 & $p=.005$ \\
\hline $\begin{array}{l}\text { Social Function } \\
\text { RAND SF-36 } 1.0\end{array}$ & $56.9 \pm 22.91$ & $70.8 \pm 20.66$ & 14.88 & $p=.003$ \\
\hline General Health & $57.6 \pm 18.13$ & $60.5 \pm 23.81$ & 2.9 & $p=.566$ \\
\hline
\end{tabular}


Table 4.12 Between Group Baseline to 6 week Treatment Outcome Differences in Disability and Health Status.

\begin{tabular}{|c|c|c|c|}
\hline Measure & $\begin{array}{l}\text { Yoga Change Score } \\
\qquad(\mathrm{n}=21)\end{array}$ & $\begin{array}{l}\text { PT Change Score } \\
(\mathrm{n}=21)\end{array}$ & $\begin{array}{c}\text { Mann } \\
\text { Whitney U } \\
p \text { Value }\end{array}$ \\
\hline & $\mathrm{M} \pm \mathrm{SD}$ & $\mathrm{M} \pm \mathrm{SD}$ & \\
\hline $\begin{array}{l}\text { RMDQ } \\
\text { RAND SF-36 } 1.0\end{array}$ & $1.57 \pm 2.76$ & $2.14 \pm 3.87$ & $p=.671$ \\
\hline $\begin{array}{l}\text { Bodily Pain } \\
\text { RAND SF-36 } 1.0\end{array}$ & $9.64 \pm 18.84$ & $20.83 \pm 21.07$ & $p=.037$ \\
\hline $\begin{array}{l}\text { Physical Function } \\
\text { RAND SF-36 } 1.0\end{array}$ & $9.75 \pm 14.18$ & $10.71 \pm 15.10$ & $p=.864$ \\
\hline $\begin{array}{l}\text { Social Function } \\
\text { RAND SF-36 } 1.0\end{array}$ & $8.33 \pm 20.28$ & $14.88 \pm 18.37$ & $p=.215$ \\
\hline General Health & $5.23 \pm 10.66$ & $2.82 \pm 16.62$ & $p=.394$ \\
\hline
\end{tabular}

$\mathrm{PT}=$ physical therapy

$\mathrm{RMDQ}=$ Roland Morris Disability Questionnaire

RAND SF-36 1.0 = Rand Short-Form 36-Item Health Survey 1.0

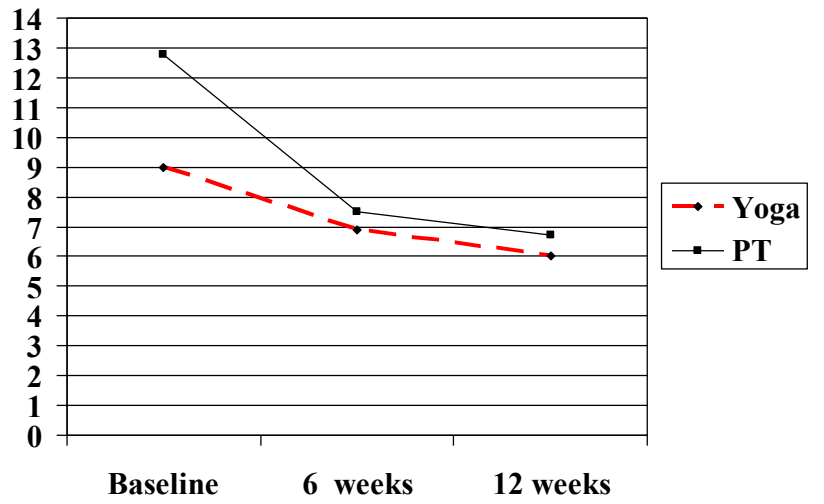

Figure 4.1 Group Differences in Average Number of Days in Pain in the Past 2 Weeks at Baseline, 6 and 12 Weeks. 
$(p \leq 0.05)$. The average number of days with reduced activity secondary to pain decreased for PT participants from $13.80 \pm 10.78$ days at baseline, to $6.95 \pm 9.05$ days at 6 weeks $(p \leq 0.01)$, declining further to $4.78 \pm 7.69$ days at 12 weeks $(p \leq 0.01)$. Between group differences in the average number of days with reduced activity due to pain were significant at each assessment period ( $p \leq 0.05)$, with yoga participants having significantly less activity limitation secondary to pain that persisted across time. Figure 4.2 shows the group differences across time in the average number of days with reduced activity secondary to pain.

Average pain bothersomeness scores in the past two weeks also improved across time for both groups. The average pain bothersomeness score decreased for yoga participants from $4.19 \pm 2.48(0-10$ scale $)$ at baseline to $3.23 \pm 2.04$ at 6 weeks $(p=0.18)$ increasing slightly to $3.42 \pm 2.67$ at 12 weeks $(p=0.24)$. Average pain bothersomeness score changes for yoga participants did not reach the criterion for clinically significant change at either 6 or 12 weeks. Average pain bothersomeness scores decreased significantly for PT participants from $7.38 \pm 1.85$ points at baseline to $3.76 \pm 2.04$ at 6 weeks $(p \leq 0.01)$ and to $3.26 \pm 2.12$ at 12 weeks $(p \leq 0.01)$. The change score differences from baseline to 6 weeks and from baseline to 12 weeks were clinically significant for the PT group where a 1.5 score change is considered clinically meaningful on an 11-point bothersomeness of pain scale (Farrar, Young, LaMoreaux, Werth, \& Poole, 2001). Between group differences in average pain bothersomeness that was significant at baseline $(p \leq 0.01)$ was not significant at 6 weeks $(p=0.67)$ or at 12 weeks $(p=0.14)$. This indicates that PT was successful in reducing average pain bothersomeness to levels similar to the yoga group by 6 and 12 weeks. Figure 4.3 shows the across time changes in average pain bothersomeness for both groups.

Within group improvements in worst pain bothersomeness in the past month were also found for both groups. Worst pain bothersomeness scores dropped for yoga participants from $6.48 \pm 2.60$ at baseline to $5.14 \pm 2.28$ at 6 weeks $(p=0.13)$ but increased slightly to $5.28 \pm 2.66$ at 12 weeks $(p=0.80)$. However, the reduction in worst pain bothersomeness at 6 and 12 weeks did not reach the 1.5 point change score of clinical significance. PT participant baseline scores decreased from $8.09 \pm 1.41$ to $6.04 \pm$ 2.85 at 6 weeks $(p \leq 0.01)$, declining further to $5.46 \pm 2.29$ at 12 weeks $(p \leq 0.01)$. The score change from baseline to 6 weeks did achieve the criterion for clinically meaningful change in worst pain bothersomeness.. The between group difference in worst pain bothersomeness in the past month was statistically significant at baseline $(p \leq 0.01)$ but not at 6 weeks $(p=0.26)$ or at 12 weeks $(p=0.79)$. The group changes in worst pain bothersomeness across time are shown in Figure 4.4.

Changes in pain medication use were compared within groups at 6 weeks using Wilcoxin Signed Ranks tests and between groups using Mann Whitney U tests. At baseline, PT participants averaged $25.7 \pm 19.87$ doses of pain medication in the past 2 weeks. At 6 weeks, the average number of doses had decreased to $14.33 \pm 14.87$ doses $(p=0.01)$. Yoga participants averaged $6.6 \pm 8.7$ doses of pain medication at baseline, which was significantly less than the PT group $(p=0.01)$. At 6 weeks, yoga participants 


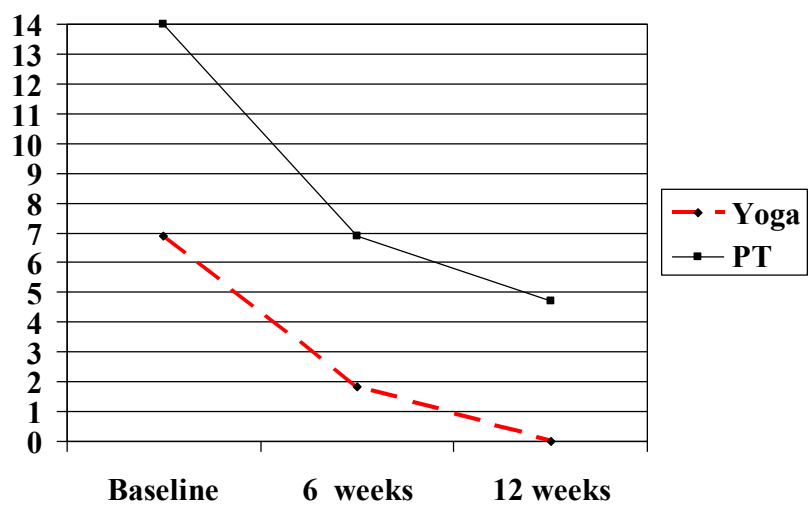

Figure 4.2 Number of Days in the Past Month with Reduced Activity Secondary to Pain.

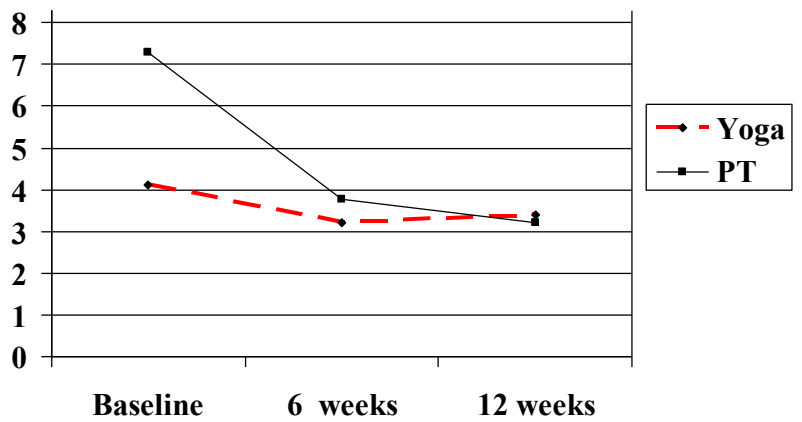

Figure 4.3 Average Pain Bothersomeness in the Past 2 Weeks.

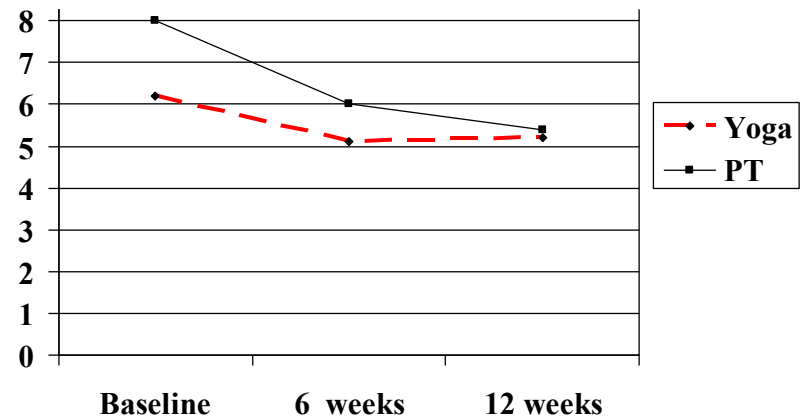

Figure 4.4 Worst Pain Bothersomeness in the Past Month. 
had decreased their average number of doses to $5 \pm 8.166$, but this decrease was not statistically significant $(p=.232)$.

Changes in pain medication use were also compared using Chi-square analysis by asking participants if they thought they were taking more, less or the same amount of pain medication at 6 and at 12 weeks compared to what they were using prior to yoga or PT. This analysis found no significant differences in changes in pain medication use between the groups. The majority of both groups indicated that they were taking less pain medication at 6 and at 12 weeks compared to their baseline use. Yoga participants also had a greater number of people reporting no change in their pain medication use, qualifying this by stating that they were not using pain medication at baseline. Group differences in pain medication use in the past two weeks are shown in Table 4.13.

Differences between the groups in treatment-related characteristics at 6 weeks were analyzed using Mann Whitney $U$ tests for continuous variables and Chi-square tests for categorical and nominal variables. A significant difference was found between the groups in the average number of hours of treatment when assessed at 6 weeks. Yoga participants averaged $10.48 \pm 1.85$ hours of class time at 6 weeks, and PT participants averaged $9.04 \pm 2.29$ hours of individualized physical therapy at 6 weeks $(p=.05)$.

There was no significant difference between the groups in the average number of minutes of weekly home practice, with both groups reporting they practiced about an hour a week. The groups also had similarly high levels of treatment satisfaction. Treatment benefits were also similar, with both groups reporting improvement in pain and pain medication usage. However, there were differences in other reported benefits. PT participants were more likely to report increased strength following treatment, while yoga participants were more likely to report improved flexibility and reduced stress. Adverse outcomes were low for both groups. One yoga participant reported increased heel spur pain that occurred during the course of yoga, and two PT participants received epidural injections while undergoing PT due to increased pain symptoms. After receiving the epidurals, both participants were able to continue PT and their home practice due to improved pain symptoms. Table 4.14 shows the differences in treatment-related characteristics at 6 weeks.

Differences between the groups in treatment-related characteristics at 12 weeks were also analyzed using Mann Whitney $U$ tests for continuous variables and chi-square tests for categorical and nominal variables. There were no significant differences between the groups at 12 weeks in minutes of home practice, treatment satisfaction or in the incidence of adverse effects. Both groups were continuing to practice at home, with yoga participants practicing an average of 50 minutes a week and PT participants practicing 77 minutes a week. Adverse outcomes reported at 12 weeks by yoga participants included persistent heel spur pain when performing standing yoga poses and increased back pain that improved with home practice performed at a gentler pace. Two PT participants who required epidural injections for increased pain that occurred during the course of PT reported continued pain relief, enabling them to continue their home 
Table 4.13 Group Differences in Pain Medication Usage at 6 and 12 Weeks.

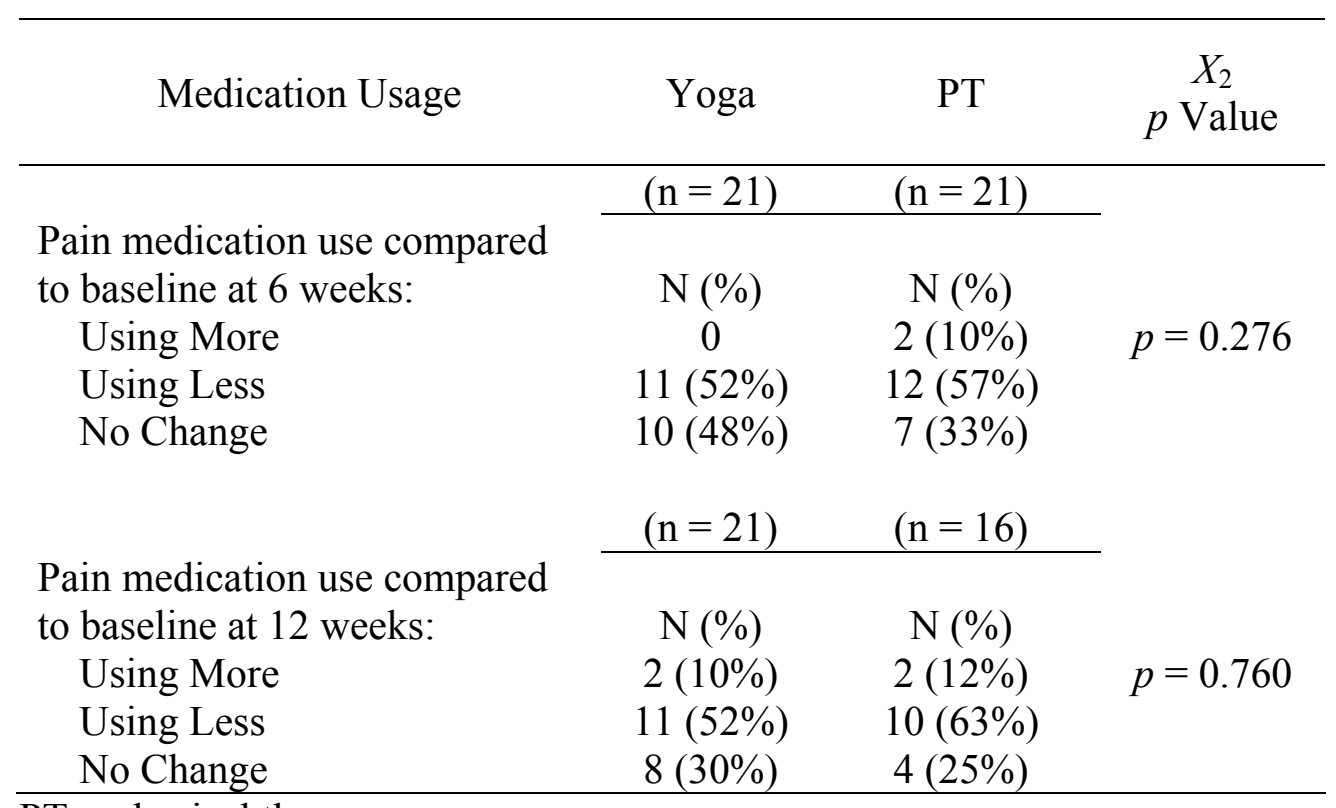

$\mathrm{PT}=$ physical therapy 
Table 4.14 Between Group Treatment Outcome Differences at 6 Weeks.

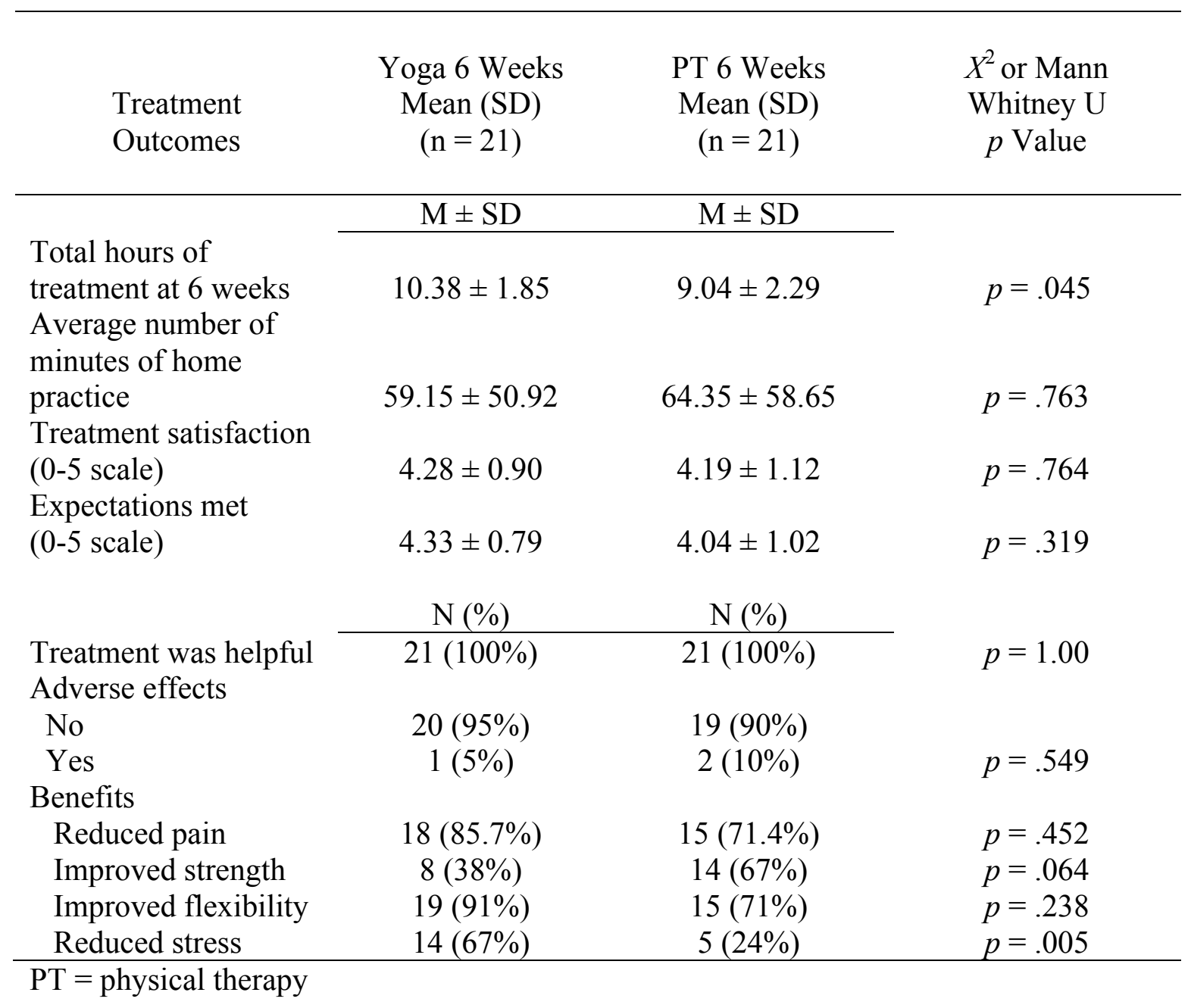


exercises at 12 weeks. Table 4.15 shows the 12 week treatment-related outcomes for both groups.

PT and yoga participants were also invited to share some of their opinions about their treatment experiences. Additional comments reported by PT and yoga participants indicated other treatment benefits and issues. One yoga participant stated, "The sharing of our experiences with our pain at the beginning of each class was very beneficial to me in helping me to not feel so alone."

Other yoga comments included, "I'm happier," "I just feel better in general," "I feel more space in my spine," "My coworkers like me better," "I have a better quality of life," "My circulation is better," and "I feel more relaxed."

Comments from PT participants included, "Therapy helped me to feel motivated to go out and do things and less depressed," "I walk better now," "My posture is improved," "My back is straighter," "My leg pain is better" and "My core is stronger."

When asked if there was anything negative about the treatment experience, two PT participants reported problems related to cost and insurance restrictions. One participant stated, "Insurance only allowed me to go to therapy for 7 visits. Going every week really helped my pain; I can tell a difference now that I'm not going to therapy any longer." Another stated, "I couldn't afford to keep going; even with insurance I had to pay about $\$ 600.00$ myself, and with three kids I just couldn't afford it any longer." 
Table 4.15 Between Group Treatment Outcome Differences at 12 Weeks.

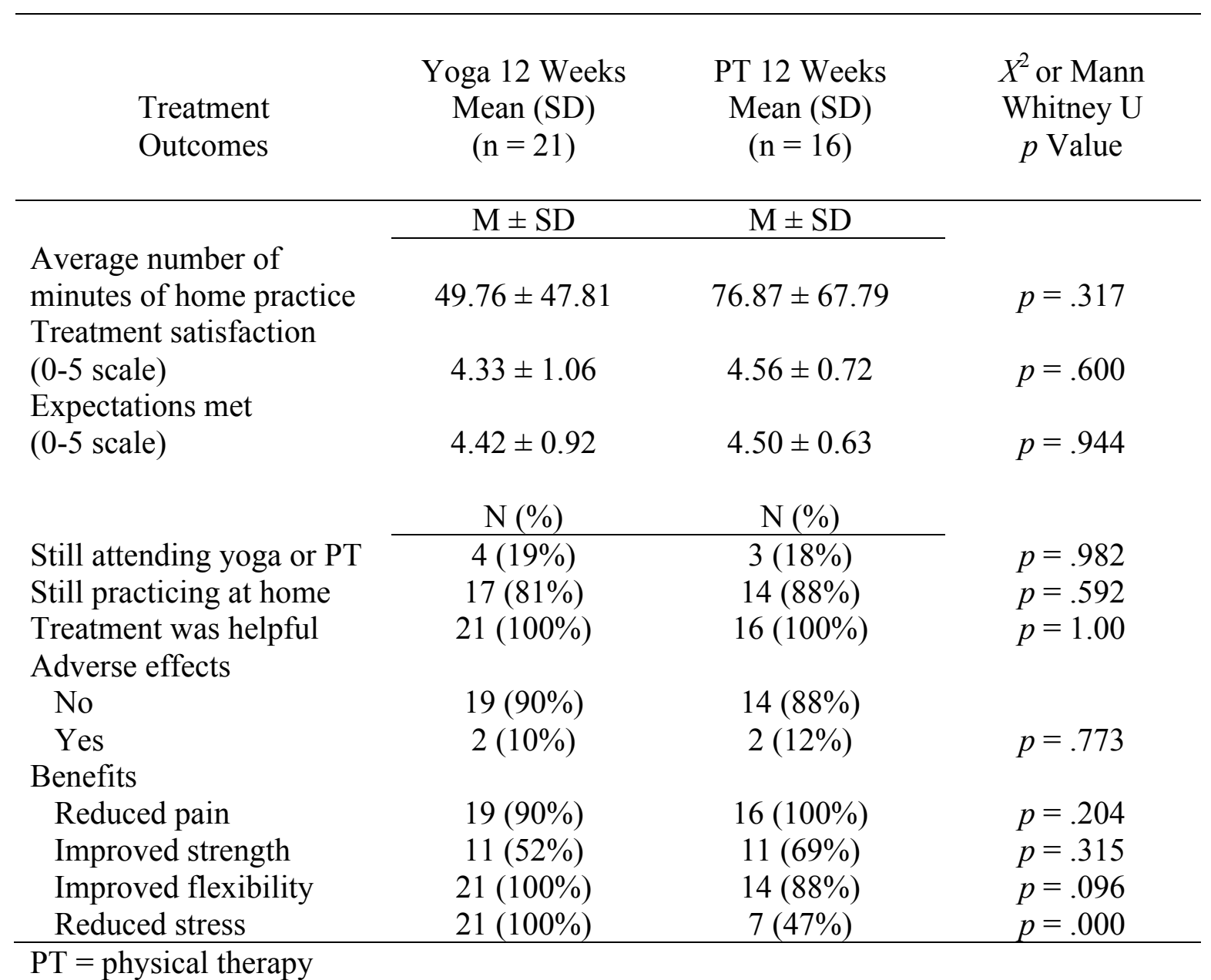




\section{CHAPTER 5. DISCUSSION}

The purpose of this study was to examine the characteristics and treatment outcomes of individuals with cLBP self-selecting yoga versus physical therapy. Chronic low back pain has been shown to be a costly and difficult to treat public health problem. Among the treatment challenges is that existing research has failed to identify patient characteristics that clearly predict improved therapeutic outcomes. As a result, treatment failures are common, and treatment failures add to costs of care. This has led to efforts to identify beneficial and cost-effective treatment approaches.

Recent evidence demonstrates that use of yoga is a low cost and beneficial approach for reducing pain and improving function in individuals with cLBP. What has not yet been studied is whether individuals with cLBP who self-select yoga differ from individuals selecting conventional therapy, like PT, and if pre-treatment differences affect treatment outcomes. This study was designed to address this gap to improve healthcare provider knowledge of the patient characterisitics associated with improved cLBP treatment outcomes following yoga or PT. The findings from this study will be organized and discussed by study aims and research questions.

\section{Aim 1-Baseline Characteristics and Differences}

A convenience sample of 53 adults, 18 years or older, volunteered to participate in the study. Statistical comparisons found no significant differences between the groups in age, gender, marital status, education or back pain etiology. The average age of study participants was $50.69 \pm 15.56$ years with an average duration of cLBP of $9.02 \pm 9.29$ years. Both groups were predominantly white, employed, college-educated, middle-class, married females. These findings are consistent with those of other yoga and exercisebased physical therapy researchers (Birdee et al., 2008; Galantino et al., 2004; Marshall \& Murphy, 2008; Saper et al., 2004; Sherman et al., 2005; Williams et al., 2005). Marshall and Murphy (2008) caution that conclusions drawn from studies that recruit cLBP volunteers to participate in exercise interventions may not generalize to other subgroups of individuals with cLBP because volunteers in exercise-based studies may anticipate improved outcomes biasing results. Additionally, those who volunteer for exercise studies may have different or fewer co-morbid conditions.

The present study found differences between the groups on psychological, disability, and health status measures. Yoga participants were significantly less disabled and had higher pain self-efficacy and health status scores at baseline compared to PT participants. These findings suggest that the individuals selecting yoga may have had greater confidence in their personal capacity to control back pain symptoms and were less functionally limited by their back pain compared to PT participants. This was supported when examining differences in pain characteristics at baseline which showed that PT participants had significantly more pain and pain medication use compared to the yoga participants. 
When comparing baseline fear of movement and depression scores, no significant differences were found between the groups. Both groups scored in the high fear of movement range with scores $\geq 38$ and in the low to normal range of depression on the Beck Depression Inventory-II. High fear of movement is a consistent finding in individuals with cLBP (Schers, Wensing, Huijsmans, van Tulder, \& Grol, 2001). The finding that neither of the groups was depressed at baseline suggests that this study's population may not be typical of other cLBP populations where depression is a commonly associated co-morbidity (Cherkin et al., 1996; Deyo et al., 1998; Thomas et al., 1999).

There also were differences between the groups on the RAND SF-36 1.0 physical function, bodily pain, social function and general health subscales. Yoga participants had significantly higher health status scores at baseline compared to the PT sample whose health status scores are more typical of the cLBP normative samples reported by other back pain researchers (Nicholas, Asghari, \& Blyth, 2008; Pahl et al., 2006), including Sherman and colleagues' (2005) yoga cLBP sample. This supports that the yoga participants in this study were less functionally impaired at baseline compared to the PT participants and other cLBP populations.

The biggest difference between the yoga and PT groups at baseline was in pain level, days in pain and activity limitation because of pain. There was no significant difference between the groups in worst pain bothersomeness in the 6 months prior to beginning treatment $8.5 \pm 1.60$ for the PT group and $7.7 \pm 2.14$ for the yoga group $(\mathrm{p}=$ 0.113 ); however, the groups had significantly different average pain bothersomeness in the two weeks prior to beginning treatment. Average pain bothersomeness for PT participants was $7.5 \pm 1.83$, similar to the worst pain bothersomeness reported by yoga participants for the period 6 months prior to treatment. This compared to the average pain bothersomeness score for the two weeks prior to treatment of $4.5 \pm 2.37$ for yoga participants $(\mathrm{p}=.000)$. These values are similar to those reported by Sherman et al., (2005), who reported average pain bothersomeness scores of $5.5 \pm 1.7$ at baseline in their cLBP sample.

Worst pain bothersomeness in the month preceding treatment was also significantly different between the groups. PT participants had a score of $8.3 \pm 1.48$, similar to their worst pain bothersomeness score 6 months prior to starting treatment compared to $6.5 \pm 2.60$ for the yoga group $(\mathrm{p} \leq 0.01)$. This suggests that PT participants were more likely to be experiencing an acute exacerbation prior to treatment while yoga participants had lower levels of pain with less functional impairment and disability.

There were also significant differences between the groups in the average number of days in pain in the previous two weeks and days with activity limitation due to pain. PT participants averaged $13.0 \pm 2.28$ days in pain in the two weeks preceding treatment compared to $9.7 \pm 5.21$ for yoga participants $(\mathrm{p}=.010)$. PT participants also reported significantly more days with activity limitation, $14.0 \pm 11.36$ days, compared to yoga participants, $8.7 \pm 10.93$ days $(\mathrm{p}=.039)$. 
Pain medication usage also differed for yoga and PT groups. At baseline, $63 \%$ of yoga participants reported use of some type of medication for pain compared to $81 \%$ of PT participants $(\mathrm{p}=.087)$. PT participants had averaged $22.2 \pm 19.80$ doses of pain medication in the two weeks prior to beginning treatment compared to $9.3 \pm 12.89$ doses for yoga participants $(\mathrm{p}=.018)$. These differences support that PT participants most likely entered treatment during an acute pain exacerbation while yoga participants entered treatment with lower levels of pain, consistent with the baseline differences in health status and disability scores observed between the groups.

The groups also differed in their treatment expectations and in their reasons for treatment selection. The most important treatment benefit for both groups was reduced pain; however, PT participants were more likely to choose reduced pain medication use as an important outcome while yoga participants were more likely to choose increased flexibility as an important outcome. PT participants also were more likely to have chosen PT based on healthcare provider referral and insurance coverage while yoga participants were more likely to have chosen yoga upon recommendation by others or from having read about it. The difference between the groups in treatment selection based on insurance coverage is an important distinction. The majority of PT participants had insurance coverage for care while the majority of individuals selecting yoga did not. This difference may have influenced treatment expectations and affected treatment outcomes. Individuals with insurance may behave or approach treatment differently than those without insurance since their choice for care is not based on the same costs or risks (http://wikipedia.org/wiki/Moral hazard). Therefore, differences in treatment outcomes between the groups may reflect differences in motivation to improve, personal accountability or locus of control that this study failed to measure.

The significant difference between the groups in selecting treatment based on healthcare provider referral vs. personal research is another important difference that may have influenced treatment motivation and outcomes. Only one yoga participant reported that their healthcare provider suggested that they consider yoga for treatment of their back pain compared to $100 \%$ of the PT participants. Despite the 2007 recommendations by the American College of Physicians and the American Pain Society that yoga be considered in the long-term management of cLBP (Chou, 2007), this difference in referral patterns to PT vs. yoga demonstrates that yoga is still not a well-accepted option for care among healthcare providers managing patients with cLBP. This shows a need for increased dissemination of yoga research findings to improve healthcare provider knowledge of the benefits of yoga in treating cLBP particularly for long-term care when insurance coverage becomes exhausted.

\section{Aim 2-To Compare Treatment Outcomes}

When the groups were compared at 6 weeks at the completion of treatment, both groups showed improvements in disability, depression and health status, with no significant differences between the groups when comparing change in depression and 
disability scores. At baseline, yoga participants had an average RMDQ disability score of $4.33 \pm 3.61$, indicating a low level of disability (scores $\leq 5$ on a $0-24$ scale); however, PT participants were significantly more disabled scoring in the moderate range of disability with an average score of $9.1 \pm 5.29$. At 6 weeks both groups had clinically and statistically significant reductions in disability with an average change score of $1.57 \pm$ 2.76 for yoga participants and $2.14 \pm 3.87$ for PT participants. This indicates that yoga and PT were both effective in reducing disability despite the baseline differences. These results are also consistent with those of Sherman et al. (2005), who reported a 2.5 point change score difference in the RMDQ following a Viniyoga intervention. This supports that modified Integral yoga is also effective in reducing cLBP disability. The improvements in depression scores in this population is also consistent with a previous study that examined the effects of Integral yoga on mood (Michels, et.al., 2006).

Fear of movement also improved for both groups at 6 weeks; however, neither group achieved the $30 \%$ change score criterion indicating clinically important improvement. This suggests that mitigating fear of movement may require a longer period of PT or yoga for change to occur.

At 6 weeks there was a significant difference between the groups in pain selfefficacy. Although both groups had improved scores at 6 weeks, the yoga group had a statistically significant increase in pain self-efficacy which was not achieved by the PT group. Yoga participants also had significantly higher pain self-efficacy at baseline. Individuals selecting yoga did so primarily based on personal research or recommendations from others. Yoga required active participation at personal expense. PT participants selected PT based primarily on physician referral and insurance coverage. PT incorporated exercise components but also individualized passive treatments. Differences between the groups in self-efficacy following treatment suggests that yoga has a stronger effect in enhancing an individual's self-efficacy beliefs compared to individualized PT. This may reflect the different cognitive effects that result from yoga or differences in self-confidence that result from a yoga practice when compared to that of PT.

Both groups also had statistically and clinically significant improvements in health status at 6 weeks. Each group had an average change score $>7$ points on the RAND SF-36 1.0 bodily pain, social function and physical function subscales that indicates clinically important improvement in individuals with cLBP following back treatment (Stewart et al., 1994; Taylor et al., 1999). These findings are consistent with those obtained by Sherman et al. (2005) using Viniyoga in treating cLBP and support use of Integral yoga and PT in improving health status outcomes.

Significant between group differences were found on the bodily pain and general health subscales of the RAND SF-36 1.0 at 6 weeks in contrast to the social function and physical function where no between group differences were found following treatment. PT participants obtained an average change score of $20.83 \pm 21.07$ on the Rand bodily pain subscale compared to the average change score of $9.64 \pm 18.84$ obtained by the yoga group $(p \leq .05)$. This difference suggests that PT had a stronger effect on pain compared to yoga. This outcome difference may be the result of the baseline differences 
between the groups in bodily pain health status where the PT group had significantly poorer status compared to the yoga group. This difference led to greater reductions in pain for the PT group since their pre-treatment pain was so much greater. The groups also had significant outcome differences on the RAND SF-36 1.0 general health subscale. Yoga participants had an average change score of $5.23 \pm 10.66$ on the general health subscale indicating clinically and statistically significant improvement; however, the PT change score of $2.82 \pm 16.62$ did not achieve either clinical or statistical significant improvement. This finding suggests that yoga may exert a stronger effect on overall health status compared to PT. Additionally, the difference in general health status at 6 weeks may have resulted from co-morbid health condition differences between the groups that this study did not control for or compare.

Pain variables also improved for each groups at 6 weeks. Both groups had significant reductions in the average number of days in pain with PT participants decreasing from $12.8 \pm 2.50$ days at baseline to $7.52 \pm 4.93$ days at 6 weeks and yoga participants decreasing from $9.70 \pm 5.21$ days at baseline to $6.90 \pm 5.18$ days at 6 weeks. Between group comparisons in the average number of days in pain that was significantly different at baseline was not statistically significant at 6 weeks. This indicates that both treatments were effective in reducing the incidence of days in pain, and PT reduced the number of days in pain to a similar level as yoga by 6 weeks.

Both groups also had significant improvements in the number of days with activity limitation secondary to pain at 6 weeks. Yoga participants decreased from $8.74 \pm$ 5.21 days at baseline to $1.85 \pm$ days at 6 weeks and PT participants decreased from 13.80 \pm 10.78 days at baseline to $6.95 \pm 9.05$ days at 6 weeks.

Average and worst pain bothersomeness scores also improved for both groups at 6 weeks. Average pain bothersomeness scores for PT participants decreased from $7.38 \pm$ 1.85 points at baseline to $3.76 \pm 2.04$ points at 6 weeks, both of which are statistically and clinically significant $(\mathrm{p} \leq .01)$. Average pain bothersomeness scores also decreased for yoga participants from $4.19 \pm 2.48$ at baseline to $3.23 \pm 2.04$ at 6 weeks; however, this change failed to reach clinical or statistical significance $(\mathrm{p}=.18)$. These findings show that Integral yoga improved average pain levels at 6 weeks, but the effect was not as great as that of PT. Similarly, the pain reduction following 6 weeks of Integral yoga was not as great as the reductions reported following 12 weeks of Viniyoga (Sherman et al., 2005) or 16 weeks of Iyengar yoga (Williams et al., 2005). The differences in pain reduction between this study and previous studies that used longer durations and other yoga methods may be the result of a dose response effect that results from longer intervals of yoga use. Had the intervention lasted for 8 weeks or longer, there may have been a dose response effect similar to that observed in these previous yoga studies.

Worst pain bothersomeness scores also declined significantly for PT participants decreasing from $8.09 \pm 1.41$ points at baseline to $6.04 \pm 2.85$ points at 6 weeks. Yoga participant scores also decreased from $6.48 \pm 2.60$ points to $5.14 \pm 2.28$ points at 6 weeks but the change was not statistically or clinically significant. Of interest was the finding that at 6 weeks there was no significant difference between the groups in average or 
worst pain bothersomeness scores; although the groups had differed significantly at baseline. These findings indicate that 6 weeks of PT reduced pain to levels similar to those of yoga participants, suggesting that PT was very effective in treating acute pain exacerbations and yoga was beneficial in improving less severe pain levels.

Pain medication use in the past 2 weeks decreased significantly for PT participants from $25.7 \pm 19.87$ doses at baseline to $14.33 \pm 14.87$ doses at 6 weeks $(\mathrm{p} \leq$ $.01)$. Yoga participants who were taking significantly less pain medication at baseline 6.6 \pm 8.7 doses also decreased their average use to $5.0 \pm 8.16$ doses at 6 weeks but the change was not statistically significant $(\mathrm{p}=.23)$. However, when asked if they were taking less pain medication at 6 weeks $52 \%$ of yoga participants and $57 \%$ of PT participants reported less use. These findings indicate that Integral yoga leads to reduced pain medication use, similar to that of Viniyoga (Sherman et al., 2005) and Iyengar yoga (Williams et al., 2005).

Comparisons of group differences in pain characteristics at 12 weeks showed similar changes for the yoga and PT groups as those obtained at 6 weeks. At 12 weeks there was no significant difference between the groups in the average number of days in pain in the past two weeks: PT $6.73 \pm 5.67$ days versus yoga $6.04 \pm 5.86$ days. There was also no significant difference between the groups in average pain bothersomeness in the past two weeks: PT $3.26 \pm 2.12$ points versus yoga $3.42 \pm 2.67$ points or in worst pain bothersomeness in the past month: PT $5.46 \pm 2.29$ points versus yoga $5.28 \pm 2.66$ points. Within group comparisons of average and worst pain bothersomeness from 6 to 12 weeks showed that PT participants achieved statistically significant declines in each of these pain variables at 12 weeks. Yoga participants had a slight increase in average and worst pain bothersomeness at 12 weeks; however, the increase was not statisitically or clinically significant. Clinically this may have represented a plateau effect in pain reduction for yoga participants. Since the study ended at 12 weeks it is not known whether the improvements experienced by both groups would have continued in a similar trend or if a plateau would have also occurred for PT participants.

At 12 weeks there remained significant differences between the groups in the number of days with limited activity secondary to pain. Yoga participants decreased from $1.85 \pm 3.10$ days at 6 weeks to less than one day $0.47 \pm 0.98$ at 12 weeks. PT participants decreased significantly from $6.95 \pm 9.05$ days at 6 weeks to $4.78 \pm 7.69$ days at 12 weeks. These changes support that both treatments resulted in persistent improvements in activity and function similar to the findings reported by Sherman et al. (2005).

Changes in pain medication use was assessed at 12 weeks by asking participants if they were taking more, less or the same amount of medication as they had been taking at baseline. The results found no significant differences between the groups in reduced pain medication usage; both groups reported taking less medication (52\% yoga versus $63 \% \mathrm{PT}$ ) at 12 weeks compared to baseline. These findings support the persistent benefits of both treatments on pain medication usage similar to that reported by Sherman et al. (2005) and by Williams et al. (2005). 
Both groups also reported continued therapeutic benefits at 6 and 12 weeks that included improved flexibility, reduced pain and less stress reported by the yoga group and increased strength and reduced pain and pain medication use reported by PT participants. Differences in reported treatment benefits were consistent with differences in pre-treatment group expectations indicating that both groups achieved the outcomes that they were anticipating. Treatment satisfaction which was high for both groups at 6 weeks ( $\geq 4$ on a $0-5$ Likert scale) persisted at $\geq 4$ at 12 weeks. Treatment satisfaction is associated with improved treatment outcomes and treatment adherence (Butler \& Johnson, 2008).

Treatment adherence which was measured by asking participants to report their average minutes of home practice at 6 and 12 weeks found that $\geq 80 \%$ of yoga and PT participants reported continued home practice averaging $59.15 \pm 50.92$ minutes a week at 6 weeks for yoga participants and $64.35 \pm 58.65$ minutes a week for PT participants at 6 weeks. At 12 weeks the groups were continuing to practice at home an average of $49.76 \pm$ 47.81 minutes a week for yoga participants versus $76.87 \pm 67.79$ minutes a week for PT participants with persistent reported benefits.

A significant difference was observed between the groups at 6 weeks when comparing the total number of hours of treatment that yoga and PT participants had averaged. Yoga participants averaged $10.38 \pm 1.85$ hours of yoga instruction while PT participants averaged $9.04 \pm 2.29$ hours of individualized therapy during the 6 week treatment interval. Comparisons of costs of care also differed significantly for yoga and PT groups and cost of care was cited as a reason for discontinuing PT prior to 6 weeks or 12 hours of therapy. The 12-hour yoga class series cost $\$ 75.00$ or $\$ 6.50$ per hour. PT costs varied, based on insurance coverage, but ranged from $\$ 35.00-\$ 73.00$ per 15 minute unit or $\$ 140.00$ - $\$ 292.00$ per hour of exercise therapy. Therapy costs were greater if additional modalities were required for relief of pain symptoms. Since both groups had improved therapeutic outcomes at 6 weeks, these findings support the use of yoga as cost effective and beneficial in treating cLBP.

\section{Strengths and Limitations}

This prospective, exploratory study was based on a non-randomized convenience sample of adult volunteers. This type of study design and sampling procedure threatens the external validity of a study and limits generalization of findings. The sample population was also found to be predominantly comprised of white, gainfully employed, college-educated, middle class, married females residing in a small urban, university community in the Southeast. This sample therefore may not be representative of other cLBP populations residing in different regions of the country or of other populations of individuals selecting yoga or PT for treatment of cLBP. However, since this study was descriptive and exploratory in nature and since there is limited understanding of how study variables are associated in these cLBP populations the sampling method was appropriate for answering the specific aims and research questions posed in this investigation. 
Another limitation of this study was that the findings were based on self-report measures and a repeated measures design. Repeated measures designs are subject to error arising from history, maturation, instrumentation and participant motivation. Findings based on use of self-report instruments and survey questionnaires are subject to response bias. However, repeated measures designs and the use of self-report measures are the standard recommended methods for assessing treatment outcomes when conducting clinical back treatment research (Deyo et al., 1998; Von Korff et al., 2000). This is because self-report measures correlate better than physical examination findings with improvements in back pain and function, and repeated measures designs reduce the risk of misinterpretation of findings resulting from the natural course of cLBP remissions and exacerbations.

Chronic low back pain remissions and exacerbations are not predictable. This means that during the course of treatment spontaneous changes in pain unrelated to treatment effects can occur. These changes can produce extraneous effects that confound the interpretation of dependent variable outcomes. For example, both groups may have had inflated treatment outcomes at 6 and 12 week resulting from the spontaneous remission of symptoms rather than resulting as a response to yoga or PT methods. The repeated measures design extending over the 12 -week time-frame was used to minimize the risk of drawing conclusions resulting from spontaneous symptom remission. Since both groups had improvements in multiple variables consistent with the variable relationships and directions predicted by the Disablement Model, the results support that PT and Integral yoga yielded beneficial treatment effects across time.

Another limitation is the lack of constancy of conditions where extraneous factors can influence treatment effects. The yoga sample in this study was recruited from five separate classes that were held between January 2008 and September 2008. All yoga classes were conducted in the same classroom and were taught by the same yoga instructor. PT participants were recruited from two separate locations and were conducted by seven different physical therapists; however, each participant had one primary therapist throughout the duration of treatment. This lack of consistency in therapists and therapy setting may also have resulted in extraneous treatment effects.

Previous investigators have discussed problems when interpreting physical therapy outcomes studies (Chou, 2005). Criticism included that physical therapy techniques and effects vary substantially based on patient needs and the skill and experience of the physical therapist. Since the physical therapy treatments used in this study were similar to those of other studies whose therapists and methods were uniquely tailored and based on patient needs, the findings from this study are consistent with the existing state of physical therapy research. The fact that a variety of exercise based methods and unique therapeutic approaches resulted in improved outcomes for PT participants despite the lack of constancy of conditions supports the beneficial effects of these PT methods on cLBP outcomes. 
Another limitation of this study is that there was no control for the use of other pain relieving therapies such as epidural anesthesia, acupuncture or massage therapy during the treatment intervention period. Two PT participants reported receiving epidurals during treatment for increased pain symptoms. Both participants reported that the epidural injections reduced their pain and allowed them to continue with PT and their home practice. Therefore, their reduced pain resulted not specifically from PT but rather to PT in addition to the epidural. Additionally, both yoga as well as PT participants continued to use pain relieving medications during treatment affecting pain and activity limitation. Although the use of these adjunctive therapies most likely affected treatment results, the primary aim of this study was to examine group characteristics and outcomes rather than to test the superiority of yoga compared to PT as a treatment for cLBP. Therefore, these participants were included in treatment outcome group analysis. The unique differences that were found between the groups, despite this limitation, provides relevant information that can be used by healthcare providers when advising or referring patient who are interested in the use of PT or yoga for treatment of cLBP.

The major strength of this study is that it is the first investigation to explore differences in characteristics of individuals with cLBP selecting yoga versus PT for treatment of cLBP. The information obtained from this study is unique and adds new knowledge in the understanding of how pre-existing differences are associated with differences in treatment outcomes. This knowledge has significant clinical practice implications.

\section{Clinical Practice Implications}

The results from this study are relevant to healthcare providers caring for individuals with cLBP_-particularly when counseling patients who express an interest in trying yoga for relief of pain. The findings from this study indicate that individuals with lower levels of back pain and disability may experience improvements in pain, mood, functional health status and disability by attending modified Integral yoga classes taught by instructors certified in teaching individuals with cLBP. These findings are consistent with those of previous yoga back pain research investigations in showing yoga to be beneficial in reducing pain and improving function in patients with cLBP (Sherman et al., 2005; Uyterhoeven, Khalsa, \& Whittemore, 2008; Williams et al., 2005).

This study found yoga to be economical when compared to the costs of PT. For example, the hourly cost of an Integral yoga class was $\$ 6.50 /$ hour compared to the hourly cost of $\$ 140.00-\$ 292.00$ for individualized PT. For patients who lack PT insurance coverage or for patients with high co-pay costs for PT care, yoga provides a cost effective alternative for improving pain and function. This finding is consistent with Saper et al., (2004), who found that $70 \%$ of yoga users reported spending little to no extra money for their yoga use.

Other benefits from yoga mentioned by participants included the support and relationships experienced within the yoga class setting. These positive group experiences 
may have contributed to the enhanced cognitive benefits observed among the yoga users. Since many individuals with cLBP experience significant social isolation and depression, a group treatment experience within a yoga class may yield even better back treatment outcomes for individuals with more severe levels of depression and disability than those individuals in the present sample.

An important consideration for healthcare providers when recommending yoga to patients with cLBP is providing advice on how to select a yoga teacher or yoga class. There are currently no national certification requirements for yoga teachers in the U.S. Consequently, individuals may teach yoga without certification or formal training in use of yoga with persons having specific medical conditions or physical limitations. Patients will benefit if cautioned to avoid enrolling in yoga classes taught by individuals who lack yoga certification and advanced training in use of yoga for patients with cLBP. This precaution can reduce back re-injury and symptom exacerbation.

Currently the clinical practice guidelines for treatment of cLBP are being updated to include the use of Viniyoga in the treatment of cLBP (Chou, 2007). The present study has found that Integral yoga methods are also safe and beneficial in reducing pain and improving function in individuals with cLBP. Viniyoga instructors, like Integral yoga instructors, must take advanced training and pass rigorous certification examinations prior to teaching patients with medical problems and physical limitations. Iyengar yoga has also been shown to be safe and effective in treating cLBP (Williams et al., 2005). Iyengar instructors are also required to pass rigorous certification examinations before being credentialed to teach or use yoga with individuals with cLBP. This suggests that healthcare providers should first consider referring patients with cLBP to Iyengar, Integral or Viniyoga instructors certified in teaching patients with cLBP.

Since a community may not have access to instructors certified in these methods patients should be counseled to discuss their health conditions with yoga instructors and to verify some type of teacher certification prior to enrolling in yoga classes. Healthcare practitioners should also query yoga instructors about their training and methods before referring patients. Another option for individuals living in areas that lack access to yoga instructors are yoga DVDs that are available online and range in cost from $\$ 15.00$ to $\$ 35.00$. Again, patients should be advised to purchase DVDs that are specifically designed for individuals with back pain limitations to avoid injury or symptom exacerbation.

Since the yoga participants in this study entered treatment with lower levels of pain and disability it is not known whether yoga methods would have resulted in similar benefits in a more disabled population more similar to the PT population in this study. However, these findings support that for individuals who are not experiencing an acute pain exacerbation yoga improves symptoms at far less cost than PT. 


\section{Theoretical Implications}

Chronic low back pain has proven to be a complex medical condition comprised of physical as well as psychological components. Evaluating back treatment outcomes within the narrow confines of the biomedical model or a psychosocial model lacking the inclusion of pathoanatomical factors is insufficient. Although the precise pathophysiologic mechanisms for the development of cLBP remain speculative, the findings from this study support the use and validity of the Disablement Model which incorporates the physiologic and psychosocial factors that can influence the progression to disability or lead to a diagnosis of cLBP (Verbrugge \& Jette, 1994). The Disablement Model was useful in selecting study variables and instruments, identifying variable relationships and measuring treatment effects.

Since conceptual models provide a context for the interpretation of research findings, concerns exist among yoga scholars about the use of western conceptual models when designing yoga research and explaining yoga effects. Critics argue that models based on the Western scientific paradigm are inadequate and fail to encompass the underlying Eastern understanding of the human system (Bossart, 2007). Classical yoga theories of anatomy, mind and emotions are not constructed or based on reductionistic models. Instead they are based on holistic models consisting of multiple interconnected dimensions that dynamically interact in ways that may not be understood from the limited context of a biomedical or psychosocial model. The results from this study support that individuals participating in yoga had unique outcome differences suggestive of a holistic effect in improving well-being as reflected in comments such as, "I just feel better overall, I am happier, my co-workers like me better". These comments suggest that yoga effects are not only associated with changes in the physical body but also with changes in mind, emotions and spiritual well-being. Further research that tests different models may improve interpreting yoga effects when compared to other types of back treatment therapies.

\section{Recommendations for Further Research}

The findings from this study support the need for continued yoga research in the use of yoga for treatment of cLBP particularly in populations of individuals who do not fit the stereotypical yoga user. Studies exploring barriers to yoga use by nonwhite and medically indigent populations are important since this study has shown that yoga is beneficial and economical in reducing pain and improving function in the treatment of cLBP. Studies examining healthcare provider barriers to yoga use are important since the current clinical practice recommendations include the use of yoga for long-term management of cLBP, and this study found that healthcare provider recommendation was rare for participants selecting yoga for treatment of cLBP.

Further research to test yoga components and to study yoga effects within new theoretical frameworks that incorporate spiritual as well as biopsychosocial outcomes may improve the understanding of yoga mechanisms of action. This knowledge will 
improve comparing yoga effects with those resulting from conventional medical therapies.

The results from this study also support the need for long-term studies to evaluate the costs and benefits associated with yoga use compared to use of conventional medical treatments for cLBP. The use of yoga for treating the symptoms of chronic medical conditions including cLBP is increasing among U.S. adults (Birdee et al., 2008; Saper et al., 2004). Given the current need for greater accountability in healthcare resource spending, long-term, cost/benefit analysis studies are essential to inform consumers and healthcare providers about safe, cost-effective, beneficial treatments for chronic health conditions. When treatment outcomes are similar, as they were in the present study, differences in costs of care over time become very important as evidence for updating clinical practice guidelines, changing insurance reimbursement practices and for improving treatment decision making by consumers and healthcare providers (Chou, 2005).

\section{Conclusions}

This study was designed to examine differences in pre-existing demographic and clinical characteristics of individuals with cLBP selecting yoga or PT for treatment of cLBP and to examine treatment outcomes. The results found that there were differences between the groups but treatment outcomes were similar. Both groups had improvements in pain and function and both were satisfied with care. The differences found between the groups in treatment expectations, reasons for selecting therapy and baseline pain and disability suggest that PT may be most appropriate for patients having acute pain exacerbation and adequate insurance coverage for costs of care. Similarities in treatment outcomes suggests that once acute pain exacerbation have been controlled the use of yoga for maintaining treatment benefits may reduce long-term costs of care and result in an enhanced sense of physical and emotional well-being for individuals with cLBP. 


\section{LIST OF REFERENCES}

Altmaier, E. M., Russell, D. W., Kao, C. F., Lehmann, T. R., \& Weinstein, J. N. (1993). Role of self-efficacy in rehabilitation outcome among chronic low back pain patients. Journal of Counseling Psychology, 40(3), 335-339.

Anderson, K. O., Dowds, B. N., Pelletz, R. E., Edwards, W. T., \& Peeters-Asdourian, C. (1995). Development and initial validation of a scale to measure self-efficacy beliefs in patients with chronic pain. Pain, 63(1), 77-84.

Andersson, G. B. (1997). The epidemiology of spinal disorders. In J. W. Frymoyer (Ed.), The adult spine: Principles and practice (2nd ed., pp. 93-141). Philadelphia: Lippincott-Raven.

Andersson, G. B. (1999). Epidemiological features of chronic low-back pain. Lancet, 354(9178), 581-585.

Asghari, A., \& Nicholas, M. K. (2001). Pain self-efficacy beliefs and pain behaviour. A prospective study. Pain, 94(1), 85-100.

Bandura, A. (1997). Self-efficacy: The exercise of control. New York: W. H. Freeman and Company.

Barnes, P. M., Powell-Griner, E., McFann, K., \& Nahin, R. L. (2004). Complementary and alternative medicine use among adults: United States, 2002. Advance Data (343), 1-19.

Beaton, D., Hogg-Johnson, S., \& Bombardier, C. (1997). Evaluating changes in health status: Reliability and responsiveness of five generic health status measures in workers with musculoskeletal disorders. Journal of Clinical Epidemiology, 50(1), 79-93.

Beck, A. T., Brown, G., \& Steer, R. (1996). Beck Depression Inventory II manual. San Antonio: The Psychological Corporation.

Benson, H. (2000). The relaxation response. New York: HarperCollins Publishers, Inc.

Beurskens, A. J., de Vet, H. C., \& Koke, A. J. (1996). Responsiveness of functional status in low back pain: A comparison of different instruments. Pain, 65(1), 7176.

Birdee, G. S., Legedza, A. T., Saper, R. B., Bertisch, S. M., Eisenberg, D. M., \& Phillips, R. S. (2008). Characteristics of yoga users: Results of a national survey. Journal of General Internal Medicine, 23(10), 1653-1658. 
Bossart, C. (2007). Yoga bodies, yoga minds: How Indian anatomies form the foundation of yoga for healing. International Journal of Yoga Therapy, (17), 27-33.

Brochu, M., Savage, P., Lee, M., Dee, J., Cress, M., Poehlman, E., Tischler, M. \& Ades, P. (2002). Effects of resistance training on physical function in older disabled women with coronary heart disease. Journal of Applied Physiology, 92, 672-678.

Brown, R., \& Gerbarg, P. L. (2005). Sudarshan Kriya Yogic breathing in the treatment of stress, anxiety, and depression: Part I- Neurophysiologic Model. Journal of Alternative \& Complementary Medicine, 11(1), 189-201.

Buchbinder, R., Jolley, D., \& Wyatt, M. (2001). Population based intervention to change back pain beliefs and disability: Three part evaluation. British Medical Journal, 322(7301), 1516-1520.

Butler, R. J., \& Johnson, W. G. (2008). Satisfaction with low back pain care. Spine J, $8(3), 510-521$.

Carey, T. S., Garrett, J. M., Jackman, A., \& Hadler, N. (1999). Recurrence and care seeking after acute back pain: Results of a long-term follow-up study. North Carolina Back Pain Project. Medical Care, 37(2), 157-164.

Carroll, J., Blansit, A., Otto, R. M., \& Wygand, J. W. (2003). The metabolic requirements of vinyasa yoga. Medicine \& Science in Sports \& Exercise, 35(Supplement 1:S155), 155.

Cherkin, D. C., Deyo, R. A., Street, J. H., \& Barlow, W. (1996). Predicting poor outcomes for back pain seen in primary care using patients' own criteria. Spine, 21(24), 2900-2907.

Chou, R. (2005). Evidence-based medicine and the challenge of low back pain: Where are we now? Pain Practice, 5(3), 153-178.

Chou, R., Qaseem, A., Snow, V., Casey, D., Cross, J. T., Jr., Shekelle, P., et al. (2007). Diagnosis and treatment of low back pain: A joint clinical practice guideline from the American College of Physicians and the American Pain Society. Annals of Internal Medicine, 147(7), 478-491.

Clay, C. C., Lloyd, L. K., Walker, J. L., Sharp, K. R., \& Pankey, R. B. (2005). The metabolic cost of hatha yoga. Journal of Strength Conditioning Research, 19(3), 604-610.

Desikachar, T. (1999). The heart of yoga: Developing a personal practice. Rochester: Inner Traditions International. 
Deyo, R. A., Battie, M., Beurskens, A. J., Bombardier, C., Croft, P., Koes, B., et al. (1998). Outcome measures for low back pain research. A proposal for standardized use. Spine, 23(18), 2003-2013.

Deyo, R. A., \& Weinstein, J. N. (2001). Low back pain. New England Journal of Medicine, 344(5), 363-370.

Dowling, D., Carter, M., \& Panico, R. A. (2008). Characteristics of people self-selecting yoga for treatment of chronic low back pain. Poster presented at the Symposium for Yoga Research and Therapy, Los Angeles, CA.

Ehrlich, G. E. (2003). Low back pain. Bulletin of the World Health Organization, 81(9), 671-676.

Eisenberg, D. M., Post, D. E., Davis, R. B., Connelly, M. T., Legedza, A. T., Hrbek, A. L., et al. (2007). Addition of choice of complementary therapies to usual care for acute low back pain: A randomized controlled trial. Spine, 32(2), 151-158.

Esch, T., Fricchione, G., \& Stefano, G. (2003). The therapeutic use of the relaxation response in stress-related diseases. Medical Science Monitor, 9, 23-34.

Esch, T., Fricchione, G., Stefano, G., \& Benson, H. (2002). Stress-related diseases: A potential role for nitric oxide. Medical Science Monitor, 8, 103-118.

Evans, C. J., Trudeau, E., Mertzanis, P., Marquis, P., Pena, B. M., Wong, J., et al. (2004). Development and validation of the Pain Treatment Satisfaction Scale (PTSS): A patient satisfaction questionnaire for use in patients with chronic or acute pain. Pain, 112(3), 254-266.

Farrar, J. T., Young, J. P., Jr., LaMoreaux, L., Werth, J. L., \& Poole, R. M. (2001). Clinical importance of changes in chronic pain intensity measured on an 11-point numerical pain rating scale. Pain, 94(2), 149-158.

Feuerstein, G. (1989). The yoga-sutra of Patanjali: A new translation and commentary. Rochester: Inner Traditions International.

Feuerstein, G. (2001). The yoga tradition: Its history, literature, philosophy and practice. Prescott: Hohm Press.

Fields, H. L., \& Martin, J. B. (2001). Pain: Pathophysiology and management. In E. Braunwald, A. L. SFauci, D. L. Kasper, L. L. Hauser, D. L. Longo \& J. L. Jameson (Eds.), Harrison's principles of internal medicine (pp. 55-59). New York: McGraw-Hill.

Graves, N., Krepcho, M., \& Mayo, H. G. (2004). Does yoga speed healing for patients with low back pain? Journal of Family Practice, 53(8), 661-662. 
Groth-Marnat, G. (1990). The handbook of psychological assessment (2nd ed.). New York: John Wiley \& Sons.

Hartranft, C. (2003). The yoga-sutra of Patanjali. Boston: Shambala Classics.

Hayden, J. A., van Tulder, M. W., Malmivaara, A., \& Koes, B. W. (2005). Exercise therapy for treatment of non-specific low back pain. Cochrane Database System Review, (3), CD000335-CD000335.

Heffernan, J. J. (2001). Low back pain. In J. Noble \& H. L. Greene (Eds.), Textbook of primary care medicine (3rd ed., pp. xxxi, 1919). St. Louis: Mosby.

Hodges, P. W., \& Moseley, G. L. (2003). Pain and motor control of the lumbopelvic region: Effect and possible mechanisms. Journal of Electromyography and Kinesiology, 13(4), 361-370.

Hodges, P. W., \& Richardson, C. A. (1996). Inefficient muscular stabilization of the lumbar spine associated with low back pain. A motor control evaluation of transversus abdominis. Spine, 21(22), 2640-2650.

Isacowitz, R. (2006). Pilates. Champaign: Human Kinetics.

Iyengar, B. (1976). Light on yoga. New York: Schocken.

Iyengar, B. (2005). Light on life. New York: Rodale, Inc.

Jackson, J. L., \& Browning, R. (2005). Impact of national low back pain guidelines on clinical practice. Southern Medical Journal, 98(2), 139-143.

Kabat-Zinn, J. (1982). An outpatient program in behavioral medicine for chronic pain patients based on the practice of mindfulness meditation: Theoretical considerations and preliminary results. General Hospital Psychiatry, 4(1), 33-47.

Kabat-Zinn, J., Lipworth, L., \& Burney, R. (1985). The clinical use of mindfulness meditation for the self-regulation of chronic pain. Journal of Behavioral Medicine, 8, 162-190.

Khalsa, S. B. (2004). Yoga as a therapeutic intervention: A bibliometric analysis of published research studies. Indian Journal of Physiology and Pharmacology, 48(3), 269-285.

Kori, S. H., Miller, R. P., \& Todd, D. D. (1990). Kinesiophobia: A new view of chronic pain behaviour. Pain Management, 3, 35-43. 
Lahad, A., Malter, A. D., Berg, A. O., \& Deyo, R. A. (1994). The effectiveness of four interventions for the prevention of low back pain. Jama, 272(16), 1286-1291.

Langer, E. J. (1989). Mindfulness. Cambridge: Da Capa Press.

Langevin, H. M., \& Sherman, K. J. (2007). Pathophysiological model for chronic low back pain integrating connective tissue and nervous system mechanisms. Medical Hypotheses, 68(1), 74-80.

Lavey, R., Sherman, T., Mueser, K. T., Osborne, D. D., Currier, M., \& Wolfe, R. (2005). The effects of yoga on mood in psychiatric inpatients. Psychiatric Rehabilitation Journal, 28(4), 399-402.

Lee, S. W., Mancuso, C. A., \& Charlson, M. E. (2004). Prospective study of new participants in a community-based mind-body training program. Journal of General Internal Medicine, 19(7), 760-765.

Levin, J., Lofland, K., Cassisi, J. E., Poreh, A., \& Blonsky, E. R. (1996). The relationship between self-efficacy and disability in chronic low back pain patients.

International Journal of Rehabilitation and Health, 2(1), 19-28.

Levin, J., Lofland, K., Cassisi, J. E., Poreh, A., \& Blonsky, E. R. (2003). Back pain selfefficacy scale. In B. Redman (Ed.), Measurement tools in patient education (2nd ed., pp. 337-340). New York: Springer Publishing Co.

Lind, B. K., Lafferty, W. E., Tyree, P. T., Sherman, K. J., Deyo, R. A., \& Cherkin, D. C. (2005). The role of alternative medical providers for the outpatient treatment of insured patients with back pain. Spine, 30(12), 1454-1459.

Luo, X., Pietrobon, R., Sun, S. X., Liu, G. G., \& Hey, L. (2004). Estimates and patterns of direct health care expenditures among individuals with back pain in the United States. Spine, 29(1), 79-86.

Marshall, P., \& Murphy, B. (2008). Self-report measures best explain changes in disability compared with physical measures after exercise rehabilitation for chronic low back pain. Spine, 33(3), 326-338.

McCracken, L. M., Vowles, K. E., \& Eccleston, C. (2005). Acceptance-based treatment for persons with complex, long standing chronic pain: A preliminary analysis of treatment outcome in comparison to a waiting phase. Behaviour Research and Therapy, 43(10), 1335-1346.

Michalsen, A., Grossman, P., Acil, A., Langhorst, J., Ludtke, R., Esch, T., et al. (2005). Rapid stress reduction and anxiolysis among distressed women as a consequence of a three-month intensive yoga program. Medical Science Monitor, 11(12), CR555-561. 
Michels, K. T., Edwards, B. S., Salstrom, S., Spears, M., \& Panico, R. A. (2006). Teaching therapeutic yoga to medical outpatients: Practice descriptions, process reflections, and preliminary outcomes. International Journal of Yoga Therapy, 16, 59-66.

Miller, B. S. (1998). Yoga Discipline of Freedom. New York: Bantam Books.

Miller, J. J., Fletcher, K., \& Kabat-Zinn, J. (1995). Three-year follow-up and clinical implications of a mindfulness meditation-based stress reduction intervention in the treatment of anxiety disorders. General Hospital Psychiatry., 17(3), 192-200.

Minvaleev, R. S., Nozdrachev, A. D., Kir'yanova, V. V., \& Ivanov, A. I. (2004). Postural influences on the hormone level in healthy subjects: I. The Cobra posture and steroid hormones. Human Physiology, 30(4), 452-456.

Nicholas, M. K., Asghari, A., \& Blyth, F. M. (2008). What do the numbers mean? Normative data in chronic pain measures. Pain, 134(1-2), 158-173.

Omoigui, S. (2007). The biochemical origin of pain-Proposing a new law of pain: The origin of all pain is inflammation and the inflammatory response. Part 1 of 3 - A unifying law of pain. Medical Hypotheses, 69, 70-82.

Ostelo, R. W. J. G., van Tulder, M. W., Vlaeyen, J. W. S., Linton, S. J., Morley, S. J., \& Assendelft, W. J. J. (2005). Behavioural treatment for chronic low-back pain. Cochrane Database Systematic Review (1), CD002014-CD002014.

Pahl, M. A., Brislin, B., Boden, S., Hilibrand, A. S., Vaccaro, A., Hanscom, B., et al. (2006). The impact of four common lumbar spine diagnoses upon overall health status. Spine Journal, 6(2), 125-130.

Patrick, D., Deyo, R., Atlas, S., Singer, D., Chapin, A., \& Keller, R. (1995). Assessing health-related quality of life in patients with sciatica. Spine, 20, 1899-1908.

Pruimboom, L., \& van Dam, A. C. (2007). Chronic pain: A non-use disease. Medical Hypotheses, 68(3), 506-511.

Puetz, T. W., O'Connor, P. J., \& Dishman, R. K. (2006). Effects of chronic exercise on feelings of energy and fatigue: A quantitative synthesis. Psychological Bulletin, 132(6), 866-876.

Radebold, A., Cholewicki, J., Polzhofer, G. K., \& Greene, H. S. (2001). Impaired postural control of the lumbar spine is associated with delayed muscle response times in patients with chronic idiopathic low back pain. Spine, 26(7), 724-730.

Rao, K. R. (2005). Perception, cognition and consciousness in classical Hindu psychology. Journal of Consciousness Studies, 12(3), 3-30. 
Richardson, C. A., Jull, G., Hodges, P. W., \& Hides, J. (1999). Therapeutic exercise for spinal segmental stabilization in low back pain. New York: Churchill Livingstone.

Riddle, D. L., Stratford, P. W., \& Binkley, J. M. (1998). Sensitivity to change of the Roland-Morris Back Pain Questionnaire: Part 2. Physical Therapy, 78(11), 11971207.

Roland, M., \& Fairbank, J. (2000). The Roland-Morris Disability Questionnaire and the Oswestry Disability Questionnaire. Spine, 25(24), 3115-3124.

Saper, R. B., Eisenberg, D. M., Davis, R. B., Culpepper, L., \& Phillips, R. S. (2004). Prevalence and patterns of adult yoga use in the United States: Results of a national survey. Alternative Therapies in Health and Medicine, 10(2), 44-49.

Satchidananda, S. (1990). The yoga sutras of Patanjali. Yogaville: Integral Yoga Publications.

Schers, H., Wensing, M., Huijsmans, Z., van Tulder, M., \& Grol, R. (2001). Implementation barriers for general practice guidelines on low back pain a qualitative study. Spine, 26(15), E348-353.

Sherman, K. J., Cherkin, D. C., Erro, J., Miglioretti, D. L., \& Deyo, R. A. (2005). Comparing yoga, exercise, and a self-care book for chronic low back pain: A randomized, controlled trial. Annals of Internal Medicine, 143(12), 849-856.

Stewart, A. L., Hays, R. D., Wells, K. B., Rogers, W. H., Spritzer, K. L., \& Greenfield, S. (1994). Long-term functioning and well-being outcomes associated with physical activity and exercise in patients with chronic conditions in the medical outcomes study. Journal of Clinical Epidemiology, 47(7), 719-730.

Stratford, P. W., Binkley, J. M., Riddle, D. L., \& Guyatt, G. H. (1998). Sensitivity to change of the Roland-Morris Back Pain Questionnaire: Part 1. Physical Therapy, 78(11), 1186-1196.

Taylor, S. J., Taylor, A. E., Foy, M. A., \& Fogg, A. J. (1999). Responsiveness of common outcome measures for patients with low back pain. Spine, 24(17), 18051812.

Teasdale, J. D., Segal, Z. V., Williams, J. M., Ridgeway, V. A., Soulsby, J. M., \& Lau, M. A. (2000). Prevention of relapse/recurrence in major depression by mindfulness-based cognitive therapy. Journal of Consulting and Clinical Psychology, 68(4), 615-623. 
Thomas, E., Silman, A. J., Croft, P. R., Papageorgiou, A. C., Jayson, M. I., \& Macfarlane, G. J. (1999). Predicting who develops chronic low back pain in primary care: A prospective study. British Medical Journal, 318(7199), 16621667.

Uyterhoeven, S., Khalsa, S. B., \& Whittemore, L. (2008). Preliminary evaluation of a yoga program for back pain. Paper presented at the Symposium on Yoga Therapy and Research.

Vad, V. B., Bhat, A. L., \& Tarabichi, Y. (2007). The role of the back rx exercise program in diskogenic low back pain: A prospective randomized trial. Archives of Physical Medicine and Rehabilitation, 88(5), 577-582.

van Geen, J. W., Edelaar, M. J., Janssen, M., \& van Eijk, J. T. (2007). The long-term effect of multidisciplinary back training: A systematic review. Spine, 32(2), 249255.

Verbrugge, L. M., \& Jette, A. M. (1994). The disablement process. Social Science and Medicine, 38(1), 1-14.

Vlaeyen, J. W., de Jong, J., Geilen, M., Heuts, P. H., \& van Breukelen, G. (2001). Graded exposure in vivo in the treatment of pain-related fear: A replicated single-case experimental design in four patients with chronic low back pain. Behavior Research and Therapy, 39(2), 151-166.

Vlaeyen, J. W., Kole-Snijders, A. M., Boeren, R. G., \& van Eek, H. (1995). Fear of movement/(re)injury in chronic low back pain and its relation to behavioral performance. Pain, 62(3), 363-372.

Waelde, L. C., Thompson, L., \& Gallagher-Thompson, D. (2004). A pilot study of a yoga and meditation intervention for dementia caregiver stress. Journal of Clinical Psychology, 60(6), 677-687.

Ware, J. (2000). SF-36 health survey update. Spine, 25(31), 3130-3139.

Webster, B. S., Courtney, T. K., Huang, Y. H., Matz, S., \& Christiani, D. C. (2006). Survey of acute low back pain management by specialty group and practice experience. Journal of Occupational and Environmental Medicine, 48(7), 723732.

Weiner, B. K. (2008). Spine update: the biopsychosocial model and spine care. Spine, 33(2), 219-223.

Wernecke, M., \& Hart, D. L. (2001). Centralization phenomenon as a prognostic factor for chronic low back pain and disability. Spine, 26(7), 758-765. 
Williams, K. A., Petronis, J., Smith, D., Goodrich, D., Wu, J., Ravi, N., et al. (2005). Effect of Iyengar yoga therapy for chronic low back pain. Pain, 115(1-2), 107117.

Woolery, A., Myers, H., Sternlieb, B., \& Zeltzer, L. (2004). A yoga intervention for young adults with elevated symptoms of depression. Alternative Therapies in Health and Medicine, 10(2), 60-63.

Zautra, A. J., Johnson, L. M., \& Davis, M. C. (2005). Positive affect as a source of resilience for women in chronic pain. Journal of Consulting and Clinical Psychology, 73(2), 212-220. 
APPENDIXES 


\title{
Appendix A. Survey Consent Form
}

\author{
University of Tennessee Health Science Center \\ College of Graduate Health Sciences \\ Memphis, Tennessee \\ Survey Consent Form
}

Evaluation of Yoga and Physical Therapy Programs for the Treatment of Chronic Back Pain

You are being given the opportunity to participate in a survey that is part of a research study.

The purpose of the study is to look at some of the characteristics and outcomes of people who enroll in yoga and compare them with people who enroll in physical therapy for treatment of chronic back pain. Dian Dowling, MSN, FNP, APRN-BC a doctoral student at the University of Tennessee Health Science Center, School of Nursing, Memphis, Tennessee will be conducting this survey and analyzing the results.

This study involves answering a series of questions which takes about 1-1 $1 / 2$ hours to complete at the beginning and end of your back yoga class series or at the beginning and end of 6 weeks of physical therapy sessions. Six weeks after you finish the second set of questions I will call you on the phone and ask you a few more questions that should take no more than 10 minutes to answer. You will also be asked to answer a brief set of questions about your back pain and weekly practice each week for 6 weeks while in physical therapy or yoga classes that will take about 5 minutes to complete. When you complete the second set of questions you will be given $\$ 10.00$ for your time and participation.

There are no risks to participating in this survey other than tiring from answering questions or becoming upset by answering questions about your back pain.

You may not experience any personal benefits from participating in the survey; however, it may help you see how your back pain changes day to day and as a result of yoga or physical therapy.

Your participating in this study is voluntary, you are free to withdraw at any time and continue with your yoga classes or physical therapy sessions.

We will keep your personal information private. Your questionnaires will be labeled with a code number instead of your name, kept in a locked cabinet and destroyed once the study is completed.

You are free to contact the researcher, Dian Dowling, with any questions that you may have about the study at 706-207-9801. 


\section{Baseline Demographic Questionnaire}

Today's date

1. Age in years

2. Gender:

- Male

a Female

3. Years of Education

4. Race:

- Caucasian

- Asian

口 Black

- Native American

a Other

5. Relationship status:

口 Married

- Single

- Co-habitating

- Divorced

口 Widowed

6. Number of children

7. With whom do you live?

a Parents or relatives

口 Parents or relatives \& children

- Spouse/partner

- Spouse/partner \& children

口 Children

- Alone

- Roommate

- Parents or relatives \& spouse \& kids 
8. What is your current annual income?

a $\$ 10,000$ or less

- $\$ 10,000-\$ 30,000$

- $\$ 30,000-\$ 50,000$

- $\$ 50,000-\$ 70,000$

- $\$ 70,000-\$ 90,000$

- 90,000 and up

9. What is your employment status now? Check all that apply.

a Not working

- Working part-time

- Working full-time

- On disability for back

- On disability for other

口 Student

10. Since there are many causes for low back pain, select the major cause of your back pain:

a Herniated disc

- Muscle strain

- Prior back surgery

- Leg pain and sciatica

- Spinal stenosis

a Spondylolithesis

口 Scoliosis

口 Previous fracture

11. Do you have any other medical conditions currently being treated by a healthcare provider?

a Yes

a No

If yes, please list them

12. Are you taking any medication for your pain at this time including herbs, prescription medication or over the counter medication?

a Yes

a No

13. On average how many doses of pain-related medication have you taken during the past 2 weeks?

14. What other types of treatments are you now using for your back pain at this time?

- Chiropractics

- Massage

- Acupuncture

प Other (please list) 
15. Have you ever had physical therapy for your back pain?

a Yes

a No

16. If yes, circle how beneficial it was in reducing pain? $(0=$ not at all $5=$ extremely $)$ $\begin{array}{llllll}0 & 1 & 2 & 3 & 4 & 5\end{array}$

17. Have you ever used yoga for your back pain?

- Yes

a No

18. If yes, circle how beneficial it was in reducing pain? $(0=$ not at all $5=$ extremely $)$

$$
\begin{array}{llllll}
0 & 1 & 2 & 3 & 4 & 5
\end{array}
$$

19. If you are taking yoga classes please select from the list below what influenced you to choose yoga for your back pain? Check all that apply.

a Cost

a Covered by my health insurance plan

口 Recommended by my doctor

a Recommended by others

口 Read about it

- Previous experience

20. What benefits do you expect from yoga? Check all that apply.

- Reduced pain

口 Greater strength

- Greater flexibility

- Reduced stress

- Less use of pain medication

口 Other (please list)

21. What is the most important benefit to you?

- Reduced pain

口 Greater strength

- Increased flexibility

a Reduced stress

- Reduced need for pain medication

口 Other (please list)

22. If you are taking physical therapy for your back pain what influenced you to choose this method? Check all that apply.

a Cost

- Covered by my health insurance plan

- Recommended by my doctor

口 Recommended by others 

- Read about it
- Previous experience

23. What benefits do you expect from physical therapy? Check all that apply.
- Reduced pain
口 Greater strength
- Increased flexibility
a Reduced stress
- Reduced need for pain medication

○ Other (please list)

24. What is the most important benefit to you?

a Reduced pain

- Greater strength and flexibility

- Reduced stress

a Less use of pain medication

a Other (please list) 


\section{Appendix C. Roland Morris Disability Questionnaire}

When your back hurts, you may find it difficult to do some things you normally do. This list contains sentences that people have used to describe themselves when they have back pain. When you read them, you may find that some stand out because they describe you today. As you read the list, think of yourself today. When you read a sentence that describes you today, place a mark next to it. If the sentence does not describe you, then leave the space blank and go on to the next one. Remember, only mark the sentence if you are sure it describes you today.

- 1. I stay home most of the time because of my back.

口 2. I change position frequently to try and get my back comfortable.

口 3. I walk more slowly than usual because of my back.

口 4. Because of my back I am not doing any of the jobs that I usually do around the house.

- 5. Because of my back, I use a handrail to get upstairs.

- 6. Because of my back, I lie down to rest more often.

口 7. Because of my back, I have to hold on to something to get out of an easy chair.

a 8. Because of my back, I try to get other people to do things for me.

- 9. I get dressed more slowly than usual because of my back.

- 10. I only stand for short periods of time because of my back.

- 11. Because of my back, I try not to bend or kneel down.

- 12. I find it difficult to get out of a chair because of my back.

口 13. My back is painful almost all the time.

- 14. I find it difficult to turn over in bed because of my back.

- 15. My appetite is not very good because of my back pain.

- 16. I have trouble putting on my socks or stockings because of the pain in my back.

- 17. I only walk short distances because of my back.

- 18. I sleep less well on my back.

- 19. Because of my back pain, I get dressed with help from someone else.

- 20. I sit down for most of the day because of my back.

- 21. I avoid heavy jobs around the house because of my back.

口 22. Because of my back pain, I am more irritable and bad tempered with people than usual.

- 23. Because of my back, I go upstairs more slowly than usual.

口 24. I stay in bed most of the time because of my back. 


\section{Appendix D. RAND Short-Form 36-Item Health Survey 1.0 Questionnaire}

1. In general, would you say your health is:

口 Excellent

- Very good

- Good

口 Fair

a Poor

2. Compared to one year ago, how would your rate your health in general now?

- Much better now than one year ago

- Somewhat better now than one year ago

a About the same

- Somewhat worse now than one year ago

a Much worse now than one year ago

The following items are about activities you might do during a typical day. Does your health now limit you in these activities? If so, how much?

3. Vigorous activities, such as running, lifting heavy objects, participating in strenuous sports

Yes, Limited a lot a Yes, Limited a Little a No, Not limited at All

4. Moderate activities, such as moving a table, pushing a vacuum cleaner, bowling, or playing golf

\Yes, Limited a lot $\quad$ Yes, Limited a Little $\square$ No, Not limited at All

5. Lifting or carrying groceries

\Yes, Limited a lot a Yes, Limited a Little a No, Not limited at All

6. Climbing several flights of stairs

$\square$ Yes, Limited a lot $\square$ Yes, Limited a Little $\quad$ No, Not limited at All

7. Climbing one flight of stairs

\Yes, Limited a lot a Yes, Limited a Little a No, Not limited at All

8. Bending, kneeling, or stooping

\Yes, Limited a lot a Yes, Limited a Little a No, Not limited at All

9. Walking more than a mile

\Yes, Limited a lot a Yes, Limited a Little a No, Not limited at All

10. Walking several blocks

\Yes, Limited a lot $\square$ Yes, Limited a Little $\square$ No, Not limited at All 
11. Walking one block

a Yes, Limited a lot

a Yes, Limited a Little

a No, Not limited at All

12. Bathing or dressing yourself

a Yes, Limited a lot

- Yes, Limited a Little

- No, Not limited at All 
During the past 4 weeks, have you had any of the following problems with your work or other regular daily activities as a result of your physical health?

13. Cut down the amount of time you spent on work or other activities

a Yes

- No

14. Accomplished less than you would like

a Yes

a No

15. Were limited in the kind of work or other activities

a Yes

a No

16. Had difficulty performing the work or other activities (for example, it took extra effort)

a Yes

a No

During the past 4 weeks, have you had any of the following problems with your work or other regular daily activities as a result of any emotional problems (such as feeling depressed or anxious)?

17. Cut down the amount of time you spent on work or other activities

a Yes

a No

18. Accomplished less than you would like

a Yes

a No

19. Didn't do work or other activities as carefully as usual

a Yes

a No

20. During the past 4 weeks, to what extent has your physical health or emotional problems interfered with your normal social activities with family, friends, neighbors, or groups?

- Not at all

- Slightly

- Moderately

- Quite a bit

口 Extremely 
21. How much bodily pain have you had during the past 4 weeks?
a None
$\checkmark \quad$ Very mild
๑ Mild
口 Moderate
a Severe
๑ Very severe

22. During the past $\mathbf{4}$ weeks, how much did pain interfere with your normal work (including both work outside the home and housework)?
口 Not at all
口 A little bit
口 Moderately
口 Quite a bit
- Extremely

These questions are about how you feel and how things have been with you during the past 4 weeks. For each question, please give the one answer that comes closest to the way you have been feeling.

How much of the time during the past 4 weeks ...

23. Did you feel full of pep?

a All of the Time

- Most of the Time

a A Good Bit of the Time

a Some of the Time

- A Little of the Time

a None of the Time

24. Have you been a very nervous person?

a All of the Time

a Most of the Time

a A Good Bit of the Time

a Some of the Time

a A Little of the Time

a None of the Time 
25. Have you felt so down in the dumps that nothing could cheer you up?

a All of the Time

a Most of the Time

a A Good Bit of the Time

a Some of the Time

- A Little of the Time

a None of the Time

26. Have you felt calm and peaceful?

a All of the Time

a Most of the Time

a A Good Bit of the Time

a Some of the Time

- A Little of the Time

a None of the Time

27. Did you have a lot of energy?

- All of the Time

a Most of the Time

a A Good Bit of the Time

a Some of the Time

a A Little of the Time

a None of the Time

28. Have you felt downhearted and blue?

- All of the Time

a Most of the Time

- A Good Bit of the Time

a Some of the Time

a A Little of the Time

a None of the Time

29. Did you feel worn out?

a All of the Time

a Most of the Time

a A Good Bit of the Time

a Some of the Time

a A Little of the Time

口 None of the Time 
30. Have you been a happy person?

a All of the Time

a Most of the Time

a A Good Bit of the Time

a Some of the Time

a A Little of the Time

a None of the Time

31. Did you feel tired?

- All of the Time

a Most of the Time

a A Good Bit of the Time

a Some of the Time

- A Little of the Time

a None of the Time

32. During the past 4 weeks, how much of the time has your physical health or emotional problems interfered with your social activities (like visiting with friends, relatives, etc.)?

a All of the time

a Most of the time

a Some of the time

- A little of the time

a None of the time

How TRUE or FALSE is each of the following statements for you.

33. I seem to get sick a little easier than other people

口 Definitely True

口 Mostly True

口 Don't Know

- Mostly False

口 Definitely False

34. I am as healthy as anybody I know

口 Definitely True

- Mostly True

口 Don't Know

a Mostly False

口 Definitely False 
35. I expect my health to get worse

- Definitely True

- Mostly True

口 Don't Know

- Mostly False

口 Definitely False

36. My health is excellent

- Definitely True

口 Mostly True

口 Don't Know

a Mostly False

Definitely False 


\section{Appendix E. Back Pain Self-Efficacy Scale}

\section{BPSES}

In the following questions we'd like to know how your back pain affects you. For each of the following questions, please circle the number from $10=$ very uncertain, $50=$ moderately certain, to $100=$ very certain which corresponds to your certainty that you can now perform the following tasks.

1. How certain are you that you can decrease your pain quite a bit?

\begin{tabular}{cccccccccc} 
Very uncertain & \multicolumn{4}{c}{ Moderately certain } & & & Very certain \\
10 & 20 & 30 & 40 & 50 & 60 & 70 & 80 & 90 & 100
\end{tabular}

2. How certain are you that you can continue most of your daily activities?

\begin{tabular}{cccccccccc} 
Very uncertain & \multicolumn{4}{c}{ Moderately certain } & & \multicolumn{2}{r}{ Very certain } \\
10 & 20 & 30 & 40 & 50 & 60 & 70 & 80 & 90 & 100
\end{tabular}

3. How certain are you that you can keep back pain from interfering with your sleep?

\begin{tabular}{cccccccccc} 
Very uncertain & \multicolumn{4}{c}{ Moderately certain } & & & Very certain \\
10 & 20 & 30 & 40 & 50 & 60 & 70 & 80 & 90 & 100
\end{tabular}

4. How certain are you that you can make a small-to-moderate reduction in your back pain by using methods other than taking extra medication?

\begin{tabular}{cccccccccc} 
Very uncertain & \multicolumn{4}{c}{ Moderately certain } & & & Very certain \\
10 & 20 & 30 & 40 & 50 & 60 & 70 & 80 & 90 & 100
\end{tabular}

5. How certain are you that you can make a large reduction in your back pain by using methods other than taking extra medication?
Very uncertain
Moderately certain
Very certain
$\begin{array}{llllllllll}10 & 20 & 30 & 40 & 50 & 60 & 70 & 80 & 90 & 100\end{array}$ 


\section{Function Subscale}

We would like to know how confident you are in performing certain daily activities. For each of the following questions, please circle the number which corresponds to your certainty that you can perform the tasks as of now, without assistive devices or help from another person. 10= very uncertain, $50=$ moderately uncertain, $100=$ very certain. Please consider what you routinely can do, not what would require a single extraordinary effort.

\section{AS OF NOW, HOW CERTAIN ARE YOU THAT YOU CAN:}

1. Walk 100 feet on flat ground in 20 seconds?

$$
\begin{array}{cccccccccc}
\text { Very uncertain } & \multicolumn{4}{c}{\text { Moderately certain }} & \multicolumn{4}{r}{\text { Very certain }} \\
10 & 20 & 30 & 40 & 50 & 60 & 70 & 80 & 90 & 100
\end{array}
$$

2. Walk 10 steps downstairs in 7 seconds?

\begin{tabular}{|llllllllll} 
Very uncertain & \multicolumn{3}{c}{ Moderately certain } & & Very certain \\
10 & 20 & 30 & 40 & 50 & 60 & 70 & 80 & 90 & 100
\end{tabular}

3. Get out of an armless chair quickly, without using your hands for support?
Very uncertain
Moderately certain
Very certain
$\begin{array}{llllllllll}10 & 20 & 30 & 40 & 50 & 60 & 70 & 80 & 90 & 100\end{array}$

4. Button and unbutton 3 medium-size buttons in a row in 12 seconds?
Very uncertain
Moderately certain
Very certain
$\begin{array}{llllllllll}10 & 20 & 30 & 40 & 50 & 60 & 70 & 80 & 90 & 100\end{array}$

5. Cut 2 bite-size pieces of meat with a knife and fork in 8 seconds?
Very uncertain
Moderately certain
Very certain
$\begin{array}{llllllllll}10 & 20 & 30 & 40 & 50 & 60 & 70 & 80 & 90 & 100\end{array}$ 
6. Turn an outdoor faucet all the way on and all the way off?

\begin{tabular}{|clllllllll} 
Very uncertain & \multicolumn{4}{c}{ Moderately certain } & & \multicolumn{2}{r}{ Very certain } \\
10 & 20 & 30 & 40 & 50 & 60 & 70 & 80 & 90 & 100
\end{tabular}

7. Scratch your upper back with both your right and left hands?

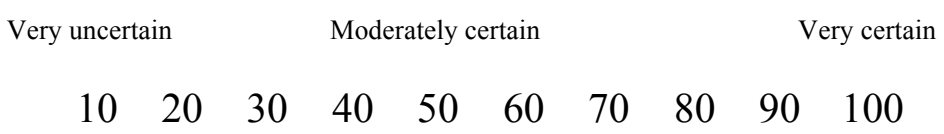

8. Get in and out of the passenger side of a car without assistance from another person and without physical aids?

\begin{tabular}{cccccccccc} 
Very uncertain & \multicolumn{9}{c}{ Moderately certain } \\
10 & 20 & 30 & 40 & 50 & 60 & 70 & 80 & 90 & 100
\end{tabular}

9. Put on a long-sleeve front-opening shirt or blouse (without buttoning) in 8 seconds?

\begin{tabular}{cccccccccc} 
Very uncertain & \multicolumn{1}{c}{ Moderately certain } & & & Very certain \\
10 & 20 & 30 & 40 & 50 & 60 & 70 & 80 & 90 & 100
\end{tabular}




\section{Other Symptoms Subscale}

In the following questions, we'd like to know how you feel about your ability to control your back pain. For each of the following questions, please circle the number which corresponds to the certainty that you can now perform the following activities or tasks. $0=$ very uncertain, $50=$ moderately certain, $100=$ very certain

1. How certain are you that you can control your fatigue?

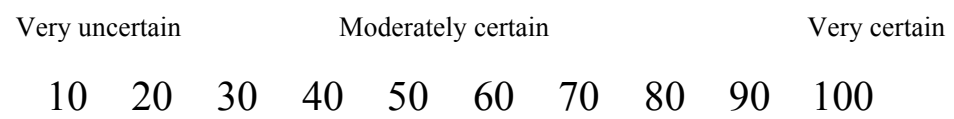

2. How certain are you that you can regulate your activity so as to be active without aggravating your back pain?
Very uncertain
Moderately certain
Very certain
$\begin{array}{llllllllll}10 & 20 & 30 & 40 & 50 & 60 & 70 & 80 & 90 & 100\end{array}$

3. How certain are you that you can do something to help yourself feel better if you are feeling blue?

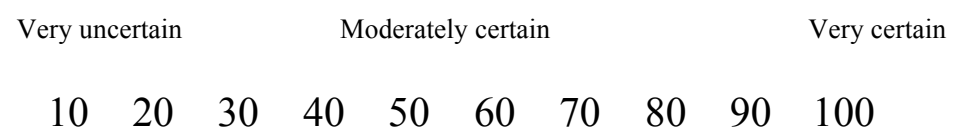

4. As compared with other people with back pain like yours, how certain are you that you can manage back pain during your daily activities?

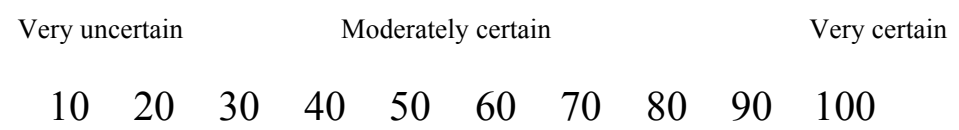

5. How certain are you that you can manage you back pain symptoms so that you can do the things you enjoy doing?

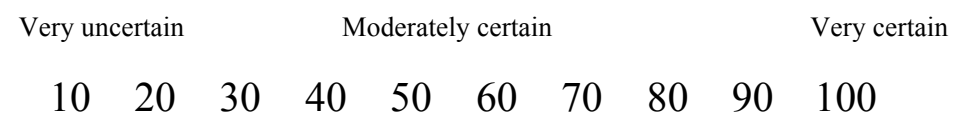

6. How certain are you that you can deal with the frustration of chronic back pain?
Very uncertain
Moderately certain
Very certain
$\begin{array}{llllllllll}10 & 20 & 30 & 40 & 50 & 60 & 70 & 80 & 90 & 100\end{array}$ 


\section{Appendix F. Tampa Scale of Kinesiophobia}

\section{TSK}

Please rate each item below according to the following scale:

1. I'm afraid that I might injure myself if I exercise

口 Strongly disagree

- Disagree

a Agree

口 Strongly agree

2. If I were to try to overcome it, my pain would increase

口 Strongly disagree

- Disagree

a Agree

\ Strongly agree

3. My body is telling me I have something dangerously wrong

- Strongly disagree

- Disagree

a Agree

\ Strongly agree

4. My pain would probably be relieved if I were to exercise

- Strongly disagree

- Disagree

- Agree

\ Strongly agree

5. People aren't taking my medical condition seriously enough

- Strongly disagree

- Disagree

- Agree

口 Strongly agree 
6. My accident has put my body at risk for the rest of my life

口 Strongly disagree

- Disagree

- Agree

- Strongly agree

7. Pain always means I have injured my body

- Strongly disagree

- Disagree

a Agree

口 Strongly agree

8. Just because something aggravates my pain does not mean it is dangerous

口 Strongly disagree

- Disagree

a Agree

\ Strongly agree

9. I am afraid that I might injure myself accidentally

口 Strongly disagree

- Disagree

- Agree

- Strongly agree

10. Simply being careful that I do not make any unnecessary movements is the safest thing I can do to prevent my pain from worsening

- Strongly disagree

- Disagree

a Agree

- Strongly agree

11. I wouldn't have this much pain if there weren't something potentially dangerous going on in my body

- Strongly disagree

- Disagree

a Agree

a Strongly agree 
12. Although my condition is painful, I would be better off if I were physically active

- Strongly disagree

- Disagree

- Agree

口 Strongly agree

13. Pain lets me know when to stop exercising so that I don't injure myself

- Strongly disagree

- Disagree

a Agree

- Strongly agree

14. It's really not safe for a person with a condition like mine to be physically active

- Strongly disagree

口 Disagree

a Agree

๑ Strongly agree

15. I can't do all the things normal people do because it's too easy for me to get injured

口 Strongly disagree

a Disagree

- Agree

口 Strongly agree

16. Even though something is causing me a lot of pain, I don't think it's actually dangerous

- Strongly disagree

口 Disagree

- Agree

- Strongly agree

17. No one should have to exercise when he/she is in pain

- Strongly disagree

- Disagree

a Agree

\ Strongly agree

Reprinted with permission. Copyright (1995). International Association for the Study of Pain. 


\section{Appendix G. Weekly Practice and Pain Log}

Date:

1. How many days have you had back pain this week?

number of days

2. On average how bothersome has your back pain been this week? Circle the number below where $0=$ not at all bothersome to $10=$ very bothersome

$\begin{array}{lllllllllll}0 & 1 & 2 & 3 & 4 & 5 & 6 & 7 & 8 & 9 & 10\end{array}$

3. What is the most bothersome your back pain has been this week? Circle the number below where $0=$ not at all bothersome to $10=$ very bothersome

$\begin{array}{lllllllllll}0 & 1 & 2 & 3 & 4 & 5 & 6 & 7 & 8 & 9 & 10\end{array}$

4. How many minutes have you practiced your exercises at home this week?

number of minutes 


\section{Appendix H. Pain Characteristics Questionnaire}

Please answer the following questions about your back pain.

1. On average, how many days during the past 2 weeks have you experienced back pain or discomfort?

2. During the past month how many days on average did you cut down on the things that you usually do for more than half the day because of low back pain? number of days

3. During the past month, how many days on average did low back pain keep you from going to work or school?

number of days

For questions 4-7 circle the number on the scale which corresponds to the bothersomeness of your back pain where $0=$ not at all bothersome to $10=$ extremely bothersome.

4. On average, during the past 2 weeks how bothersome has your back pain been?

$$
\begin{array}{lllllllllll}
0 & 1 & 2 & 3 & 4 & 5 & 6 & 7 & 8 & 9 & 10
\end{array}
$$

5. How would you rate the bothersomeness of your pain today?

$$
\begin{array}{lllllllllll}
0 & 1 & 2 & 3 & 4 & 5 & 6 & 7 & 8 & 9 & 10
\end{array}
$$

6. In the past month how bothersome was your worst pain?

$$
\begin{array}{lllllllllll}
0 & 1 & 2 & 3 & 4 & 5 & 6 & 7 & 8 & 9 & 10
\end{array}
$$

7. In the past 6 months how bothersome was your worst pain?

$\begin{array}{lllllllllll}0 & 1 & 2 & 3 & 4 & 5 & 6 & 7 & 8 & 9 & 10\end{array}$




\section{Appendix I. Short Term Yoga Program Follow-up Questionnaire}

1. Do you think that yoga has been helpful for your back pain?

a Yes

a No

2. If yes, how has it helped?

- Reduced pain

口 Improved flexibility

Increased strength

- Reduced stress

- Other benefits (please list)

3. Do you think yoga has reduced your use of medications for pain?

a Yes

№

4. Compared to your medication use listed at the beginning of your treatment have you taken

- More medication for pain relief during the past 2 weeks

- Less medication for pain relief during the past 2 weeks

a The same amount of medication for pain relief during the past 2 weeks

5. On average, how many doses of pain medication have you taken in the past 2 weeks

6. Would you recommend yoga for the treatment of back pain to others?

a Yes

№

7. Have you experienced any negative effects from yoga/ use?

a Yes

a No 
8. If yes, what did you experience?

- Increased pain

a Reduced mobility

- Increased need for pain medication

- Increased doctor visits

9. On the following scale where $0=$ not at all satisfied and $5=$ very satisfied, circle the number to answer how satisfied you have been with yoga in relieving your back pain?

$\begin{array}{llllll}0 & 1 & 2 & 3 & 4 & 5\end{array}$

10. On the following scale, where $0=$ not at all met and $5=$ met completely, circle the number to answer were the expectations and benefits that you expected from yoga met?

$\begin{array}{llllll}0 & 1 & 2 & 3 & 4 & 5\end{array}$




\section{Appendix J. Short Term Physical Therapy Follow-up Questionnaire}

1. Do you think that physical therapy has been helpful for your back pain?

a Yes

a No

2. If yes, how has it helped?

- Reduced pain

口 Improved flexibility

Increased strength

- Reduced stress

- Other benefits (please list)

3. Do you think physical therapy has reduced your use of medications for pain?

a Yes

№

4. Compared to your medication use listed at the beginning of your treatment have you taken

a More medication for pain relief during the past 2 weeks

- Less medication for pain relief during the past 2 weeks

- The same amount of medication for pain relief during the past 2 weeks

5. On average, how many doses of pain medication have you taken in the past 2 weeks?

6. Would you recommend physical therapy for the treatment of back pain to others?

a Yes

№

7. Have you experienced any negative effects from physical therapy use?

a Yes

№ 
8. If yes, what did you experience?

- Increased pain

- Reduced mobility

口 Increased need for pain medication

- Increased doctor visits

9. On the following scale where $0=$ not at all satisfied and $5=$ very satisfied, how satisfied you have been with physical therapy in relieving your back pain?

$\begin{array}{llllll}0 & 1 & 2 & 3 & 4 & 5\end{array}$

10. On the following scale, where $0=$ not at all met and $5=$ met completely, were the expectations and benefits that you expected from physical therapy met?

$\begin{array}{llllll}0 & 1 & 2 & 3 & 4 & 5\end{array}$




\section{Appendix K. Long Term Yoga and Physical Therapy Follow-up Questionnaire}

1. Are you still taking yoga classes or attending physical therapy?

- Yes

a No

2. Are you still doing your home practice?

a $\quad$ Yes

口 No

3. If yes, on average how many minutes do you practice a week?

4. Do you think that yoga or physical therapy has been helpful for your back pain?

a Yes

- No

5. If yes, how has it helped?

- Reduced pain

- Increased strength

- Improved flexibility

口 Reduced stress

口 Other benefits (list)

6. Do you think yoga or physical therapy has reduced your use of medications for pain?

a Yes

๑ No

7. Compared to your medication use listed at the beginning of your treatment you taken

- More medication for pain relief during the past 2 weeks

- Less medication for pain relief during the past 2 weeks

- The same amount of medication for pain relief during the past 2 weeks

8. Do you think that yoga/physical therapy has decreased the number of visits you make to a health care provider including chiropractors, acupuncturists or massage therapists for back pain? 
a Yes

๑ No

9. Would you recommend yoga or physical therapy for the treatment of back pain to others?

a Yes

a No

10. Have you experienced any negative effects from yoga or physical therapy use?

a Yes

a No

11. If yes, what did you experience?

12. Overall, were you satisfied with yoga/physical therapy as a treatment for your back pain?

a Yes

a No

13. If yes, how satisfied? $(0=$ not at all satisfied $5=$ very satisfied $)$

$\begin{array}{llllll}0 & 1 & 2 & 3 & 4 & 5\end{array}$

14. On the following scale, where $0=$ not at all met and $5=$ met completely, were the expectations and benefits that you expected from physical therapy met?

$\begin{array}{llllll}0 & 1 & 2 & 3 & 4 & 5\end{array}$




\section{Appendix L. Back Yoga Sequence}

\section{A Practice for Low Back Care}

1. Body Scan, awareness practice,

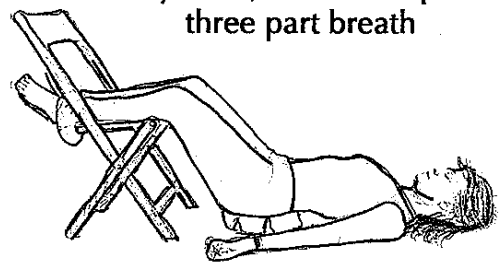

2. Pavanamuktasana (knees to chest)

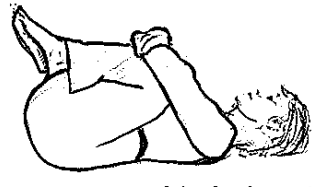

Variation 1

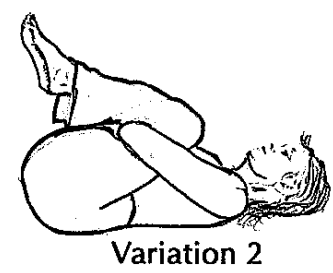

To protect the knees
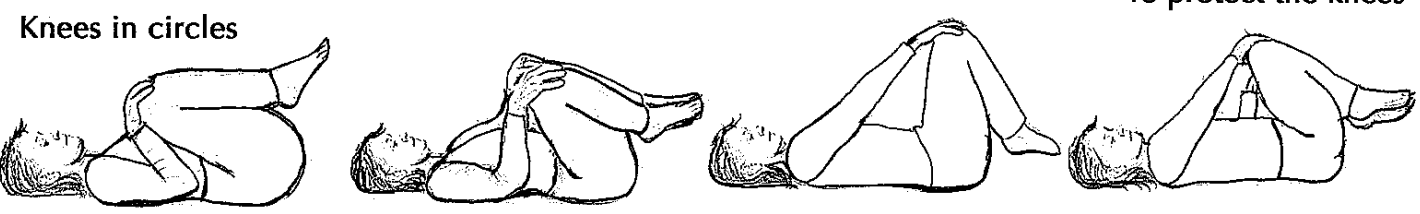

Knees to chest - rock the body from side to side place the hands on the knees ad make large slow circles

4. PNF technique

(Proprioceptive Neuromuscular Facilitation)
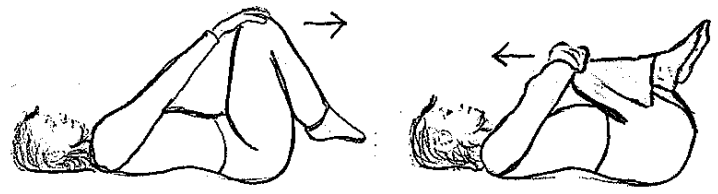

6. Setu Bandhasana (rolling bridge)

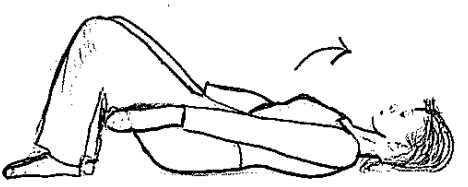

Rolling up inhale lifting both armes - hold and breathe Rolling down exhale lower both arms relax

7. Pavanamuktasana (knees to chest)

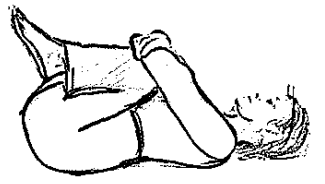

Use either variation
8. Savasana (relaxation pose)

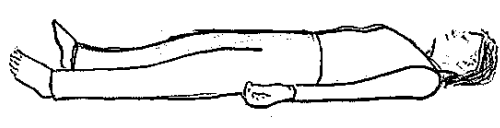

5. Pelvic tilts
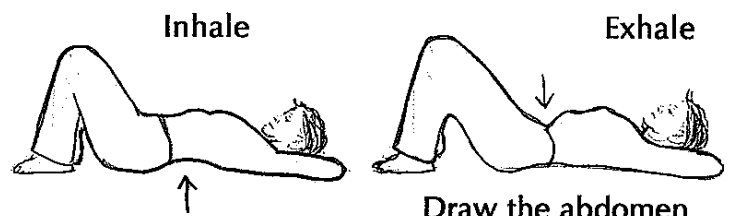

Draw the abdomen towards the spine

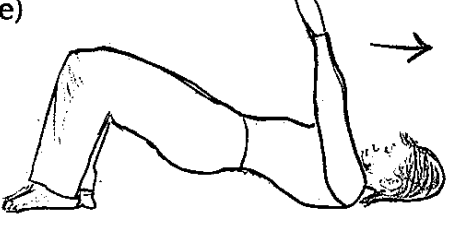

$$
\text { ax }
$$


10. Chakravakasana (dynamic sunbird pose)
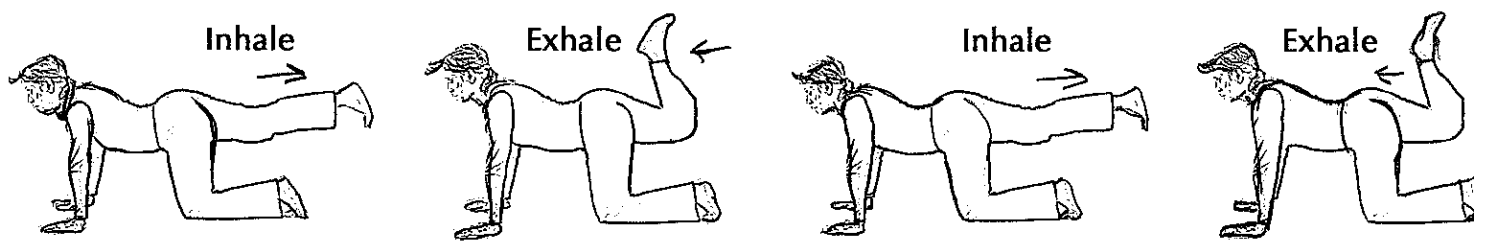

Keep strong arms and upper body. Focus on the gluteus muscles

11. Dynamic Balasana

$$
\text { Exhale }
$$
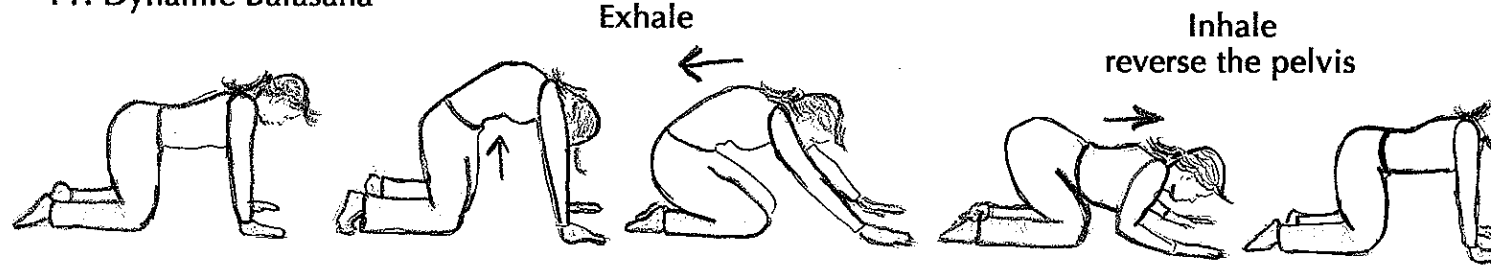

On the last round sit back into childs pose

12. Bhujangasana (cobra)

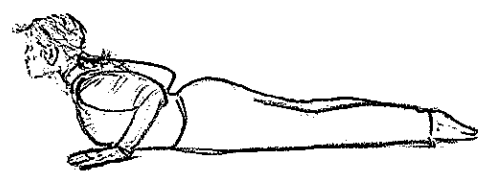

13. Ardha Salabasana (the half locust pose)
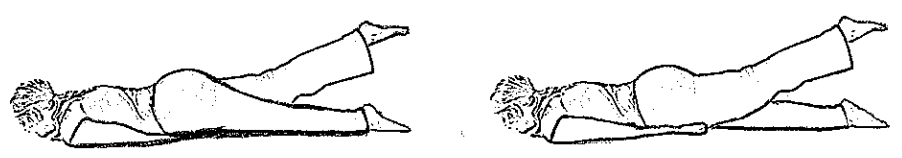

When lifting the right leg hold for several breaths then swing the leg to the right without lifting the left side of the pelvi: Center the leg, lift higher, lower the leg and reverse

14. Dynamic Forward Bend from diamond pose
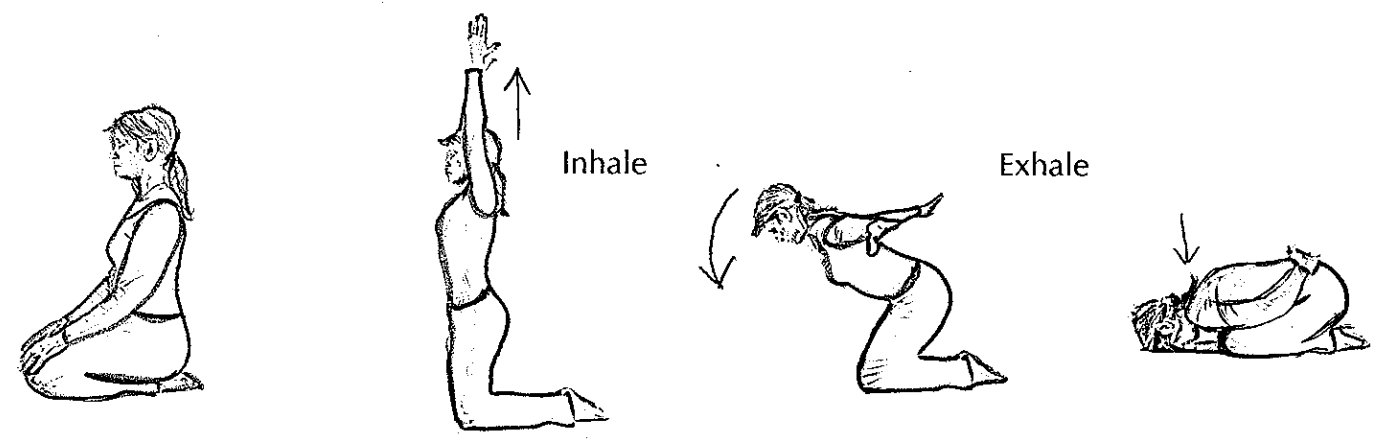
page 3

15. Dynamic Parsvotanasana (standing forward bend)
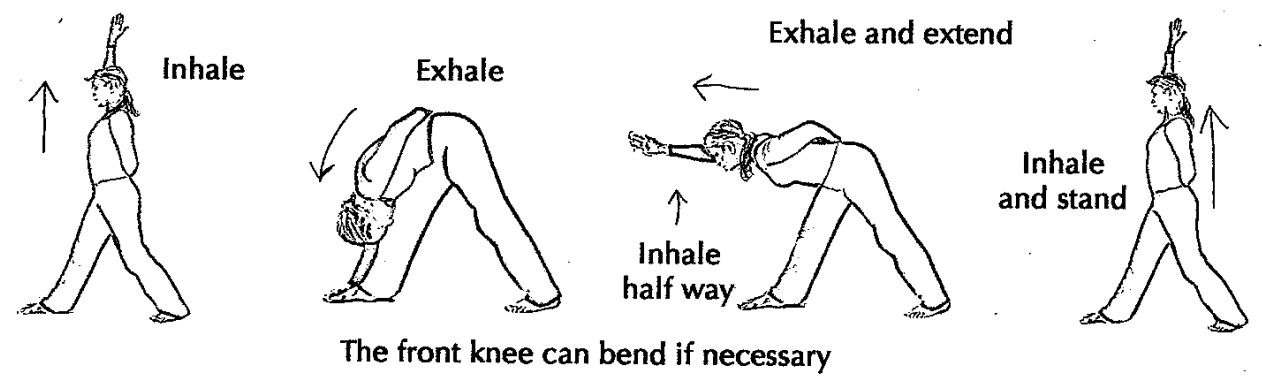

16. Supine dynamic spinal twist
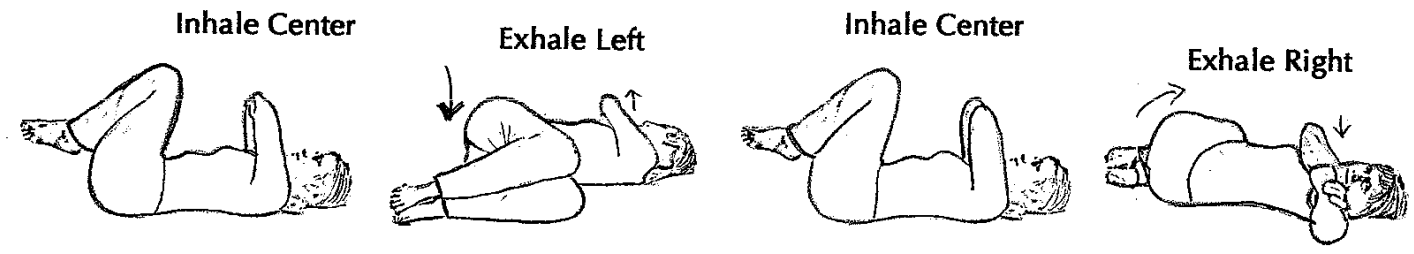

Use slow, smooth movements

17. Yoga Mudra (yogic seal)
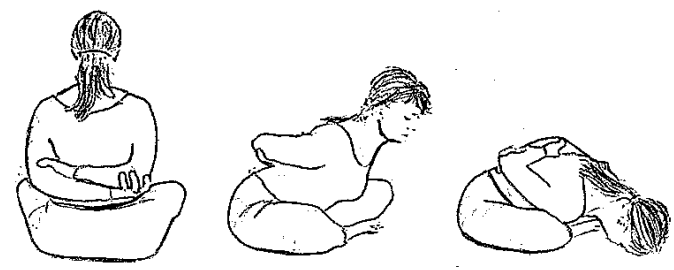

18. Yoga Nidra (deep relaxation)

20. Pranayama (breathing practices)
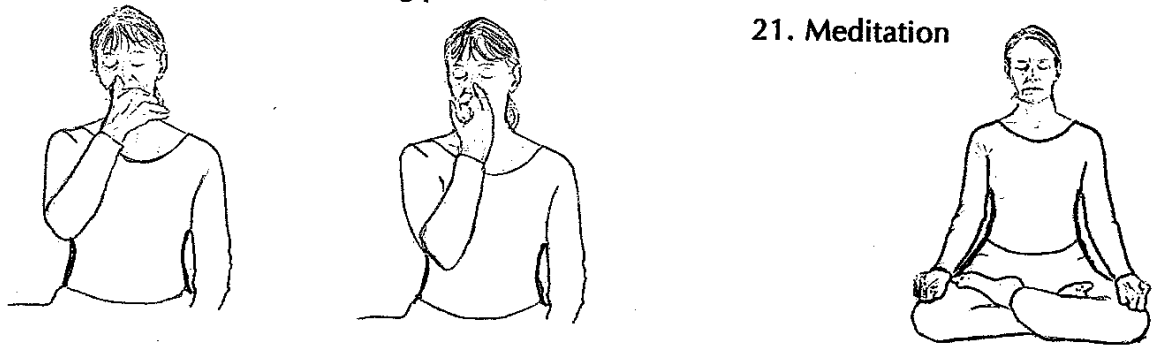

111 
Jan.31. 2008 2:13PM Pedatric parthers llo No.4663 P. 1

\section{- Physicians Back and Neck Clinic}

CHRISTOPHER E. DOERR, D.O.

ALICE WOODRUFF, APRN

JENNIFER MORLOCK, PT

TERESA MORNEAULT, PT

DEANNA LONG, PT

October 30,2007

Dian Dowling, MSN, FNP, APRN-BC

University of Tennessee Health Science Center-Memphis

College of Nursing

877 Madison

Memphis, TN 38163

Dear Ms. Dowling:

I am in receipt of your proposal "Evaluation of Yoga and Physical Therapy Programs for the Treatment of Chronic Low Back Pain". You will be conducting your study at the Athens Regional Mind Body Institute and the Physician's Back and Neck Clinic. The purpose of the study is to evaluate outcomes from a specially developed physical therapy program and a yoga program for treatment of chronic lower back pain. You will examine clinical outcomes including back pain, depression, health status, self-efficacy, fear of movement and demographic characteristics of participants to determine whether there are any differences between the groups before and after they complete the programs.

Your request to conduct the study at Physician's Back and Neck Clinic has been approved. Please inform us if there will be any changes in your study during the course of the research.

Sincerely,

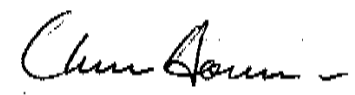

Christopher E. Doerr, D.O.

Medical Director, Physician's Back and Neck Clinic 


\section{Athens Regional}

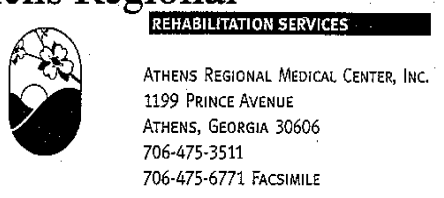

February 11,2008

Dian Dowling, MSN, FNP, APRN-BC

University of Tennessee Health Science Center-Memphis

College of Nursing

877 Madison

Memphis, TN 38163

Dear Ms. Dowling:

This letter follows up on our discussion on February $7^{\text {th }} 2008$ concerning your desire to recruit participants for your dissertation research from our patient population. I am in receipt of your proposal "Evaluation of Yoga and Physical Therapy Programs for the Treatment of Chronic Low Back Pain". I understand that this project has been approved by the University of Tennessee Health Science Center and Athens Regional Medical Center's IRB committees. You will be conducting your study at the Athens Regional Mind Body Institute, the Physician's Back and Neck Clinic and propose to add our Physical Therapy Clinic to-your research protocol. The purpose of your study is to evaluate outcomes from physical therapy programs and a yoga program for treatment of chronic lower back pain. You will examine clinical outcomes including back pain, depression, health status, self-efficacy, fear of movement and demographic characteristics of participants to determine whether there are any differences between the groups before and after they complete the programs.

Your request to conduct the study at Athens Regional Medical Center's Rehabilitation Department has been approved. Please inform us if there will be any changes in your study during the course of the research.

Sincerely,

Todd Story,

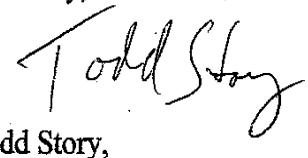

Director, Athens Regional Medical Center Rehabilitation Department 
Athens Regional

February 25,2008

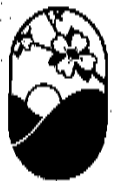

MEDICAL GENTER

Dian Dowling, MSN, FNP, APRN-BC

Medical Stafi Services

1199 Pkinge AvenuE

HTHENS, GEORGIA 30606-2793

University of Tennessee College of Nursing

$706-475-6971$

877 Madison

Memphis, Tenncssee 38163

Dear Ms. Dowling:

I am in rcceipt of a letter forwarded from Todd Story, Director of the ARMC Rehabilitation Department, in regards to your study litled "Evaluation of Yoga and Physical Therapy Programs for the Treatment of Chronic Lower Back Pain". Your initial request involved conducting your study at the Athens Regional Medical Center Mind Body Institute and the Physician's Back and Neck Clinic. The purpose of the study is to cvaluate a specially developed physical therapy program for treatment of chronic lower back pain. You will also examine clinical outcomes including back pain, depression, hcalth status, self-efficacy and fear of movement at the conclusion of the treatment programs to detemine whether there are any differences between the two groups.

I understand that you wish to include the ARMC's Physical Therapy Clinic to your rescarch protocol. Mr. Story has approved your request to have the Rehabilitation Department participate in your study.

The IRB will make this change to your original request and again pleasc be reminded should any part of your submitted proposal change at any time during the course of rescarch, please nolify the Institutional Review Committee.

We appreciate your interest in participating in the programs provided by Athetis Regional Medical Center.

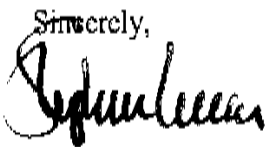

Stephen K. Lucas, M.D.

Chairman, $\mathbb{R B}$, Athens Regional

cc: Todd Story, Director, ARMC Rehabilitation Department 


\section{FAX COVER SHEET}

\section{INSTITUTIONAL REVIEW BOARD \\ THE UNIVERSITY OF TENNESSEE HEALTH SCIENCE CENTER \\ 910 MADISON AVENUE, SUITE 600 \\ MEMPHIS, TN 38163 \\ TELEPHONE: 901-448-4824 \\ FAX: 901-448-5103}

DATE: 15 December 2008

FAX NUMBER: $\quad 888-553-0990$

TO: Dian Dowling

FROM:_ Holly Herron

UTHSC IRB

SUBJECT: _study \#5111

MESSAGE Should you require any additional information, con act me at the number

listed above or at hherron@utmemedu.

No. of Pages (including cover sheet): 
March 11, 2008

Dian Dowling, MSN, FNP, APRN-BC

College of Graduate Health Sciences

Department of Nursing

Alexander Building

UTHSC

CAMPUS

Dear Ms. Dowling,

The Administrative Section of the UTHSC Institutional Review Board (IRB) reviewed your application for revision of your previously approved project entitled, "Evaluation of Yoga and Physical Therapy Programs for the Treatment of Chronic Low Back Pain".

The Administrative Section of the IRB determined your application to be consistent with the guidelines for exempt status under 45CFR46.110 (b) (2). Therefore, the attached revisions to your project were approved in this regard as complying with proper consideration of the rights and welfare of human subjects, the risk involved, and the potential benefits of the study.

In the event that volunteers, either subjects or patients, are to be recruited by means other than usual and standard patient care practices, the Board must approve of any such solicitation materials (i.e., advertising copies or posters, etc.)

Any alterations (revisions) in the research project must be submitted to and approved by the UTHSC Institutional Review Board prior to implementation to these revisions.

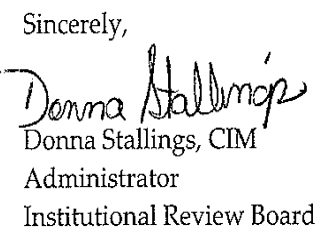

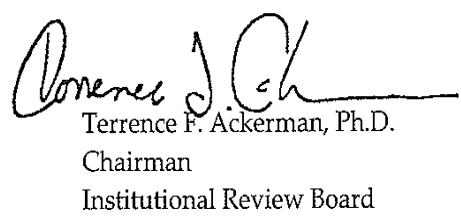

Institutional Review Board

attachment 


\section{VITA}

Dian Dowling Evans was born in Pittsburgh, Pennsylvania, on August 10, 1953. She attended the University of Georgia, graduating Magna Cum Laude in 1975 with a Bachelor of Science in Home Economics with an emphasis in Child and Family Development. In June of 1978 she completed a Master of Science from the University of Georgia in psychology with an emphasis in clinical child psychology. Her thesis research evaluated the effects of a behavior modification therapy for treating children with attention deficit disorder and was later published as a chapter in Lahey, B. (Ed.), Behavior therapy with hyperactive and learning disabled children. (49-60). New York: Oxford University Press.

After working several years as a psychologist with adults with disabilities Dian entered the Medical College of Georgia School of Nursing, graduating Summa Cum Laude in 1985 with a Bachelor of Science in Nursing. While working as a nurse, she became interested in holistic nursing and the use of complementary and alternative medicine techniques in the hospital setting. In 1989 she was selected to receive the prestigious Woodruff Fellowship by the Nell Hodgson School of Nursing at Emory University. In 1990 she graduated Summa Cum Laude from Emory as a Woodruff Fellow with a Master of Science in Nursing as a Family Nurse Practitioner. Her thesis research, a descriptive analysis of emergency department census trends and implications for nurse practitioner staffing, was published in the Journal of Nursing Management (1995, 6, 48C-48J) and the Journal of Emergency Nursing (1993, 19, 16).

Dian is currently an ANCC advanced practice family nurse practitioner working in the emergency medicine department at Emory University Hospital. She is also an assistant clinical professor in the Byrdine F. Lewis College of Nursing at Georgia State University, where she teaches undergraduate and graduate level clinical courses. She is a member of the American Nurses Association, the Beta Omnicron and Epsilon Alpha chapters of Sigma Theta Tau, the International Association of Yoga Therapists, the American Holistic Nursing Association and the Southeast Iyengar Yoga Association. She is a wife, mother and grandmother and has been studying and practicing yoga methods for the past 12 years. 